\title{
THE IMPACTS OF WORKING WITH PEOPLE EXPERIENCING SUICIDAL IDEATION: MENTAL HEALTH NURSES DESCRIBE THEIR EXPERIENCES
}

\author{
By \\ Lynley Murtagh
}

A thesis submitted to Victoria University of Wellington in partial fulfilment of the requirements for the degree of Master of Arts (Applied) in Nursing

Victoria University of Wellington

2008 


\section{Abstract}

New Zealand has one of the highest suicide rates in the world and mental health nurses are the most likely professionals to assess a person presenting with suicidal ideation. Managing a suicidal crisis is acknowledged as being one of the most difficult and frightening challenges facing mental health professionals.

This research aimed to have mental health nurses who work in acute mental health settings describe the impact that working with people experiencing suicidal ideation has on their personal and professional lives.

This study followed the tenets of fundamental qualitative description as presented by Sandelowski (2000). Five mental health nurses participated in individual semi structured interviews. The data collected under-went systematic thematic analysis and the extracted findings were presented as a straight description.

The findings from this study revealed that personal philosophies of care, the work place culture, organisational and professional expectations and their personal concepts about suicide all influenced the experiences of these participants. Mental exhaustion, tension and feelings of isolation and alienation from family and society were universal experiences.

Two recommendations have been made based on the insights gained from this research. These are; tertiary institutions should offer post graduate studies on the subject of suicide as it relates to mental health nursing and national guidelines for the provision of supervision to mental health nurses need to be developed.

Key words

Mental health-psychiatric-nursing -philosophy-models of care -suicidal ideation- work place stress. 


\section{Acknowledgements}

I would like to take this opportunity to acknowledge those who have supported me throughout this process.

I feel a special sense of gratitude toward my supervisor Cheryle Moss, for keeping faith and steering me through what proved to be a long and arduous process. My thanks also go to Jo Walton for stepping in as my supervisor and for championing me toward the finishing post. Thanks as well to those other members of the faculty at Victoria who gave so freely of themselves at different times.

I feel especially lucky to have had friends and family in my life who didn't give up on me. You made it possible for me to keep going. Thanks to Meg, Mat, Mal, Tania, Clare, Kerry, Emily and also to my new friends in Nelson.

This was especially so in regard to Suzy. Her attention to detail can be seen in the formatting of this work. Thanks for sharing your keen mind, your sense of humour, your family and your hospitality.

My appreciation also goes to the many people who have been thrust into relationship with me and who have let me go along with them as they explored the places that very few are privileged to go to with another human being.

Ian, you taught me about anger and forgiveness and made me want to try to understand. I keep you with me. 


\section{Table of Contents}

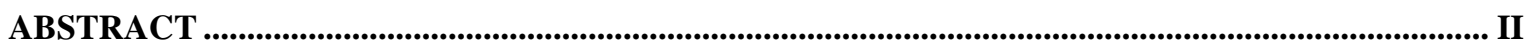

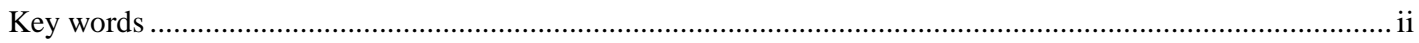

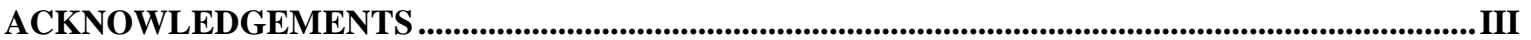

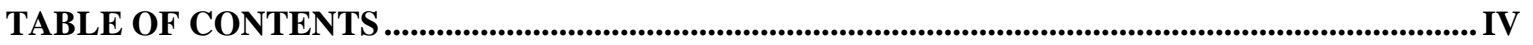

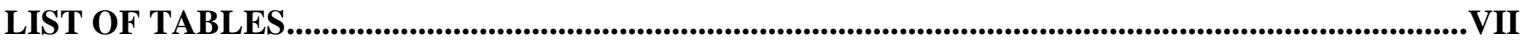

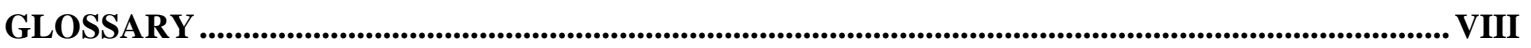

CHAPTER ONE: INTRODUCTION

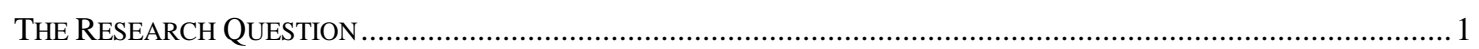

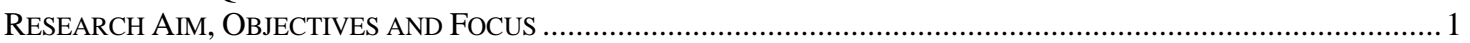

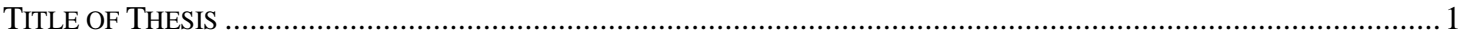

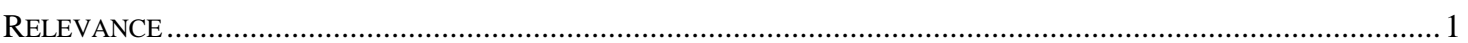

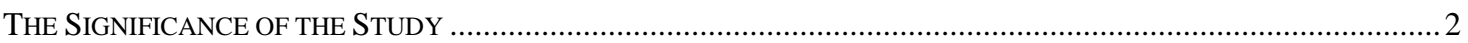

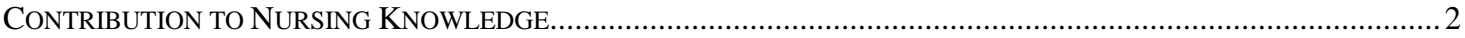

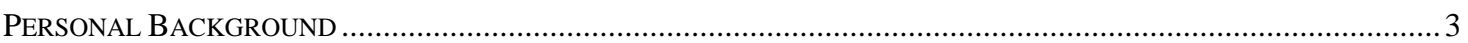

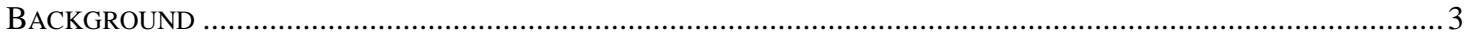

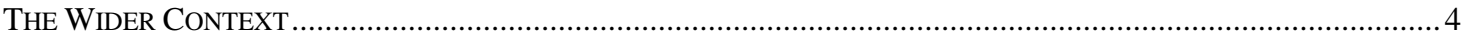

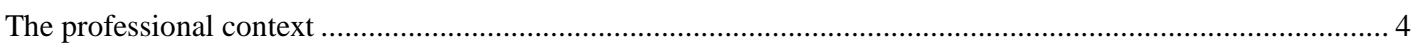

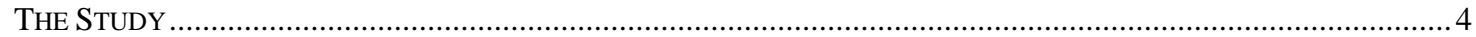

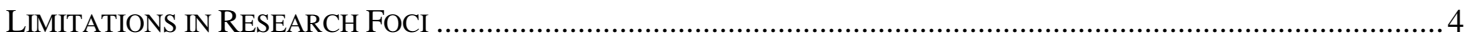

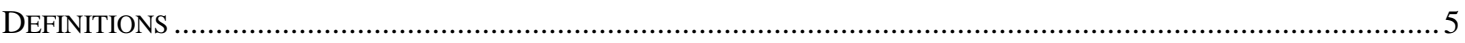

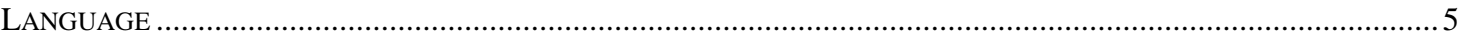

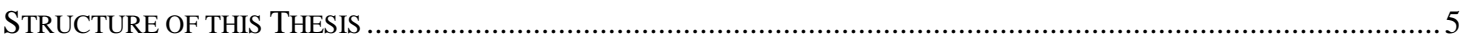

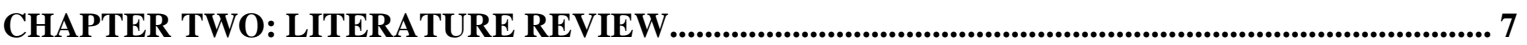

INTRODUCTION …

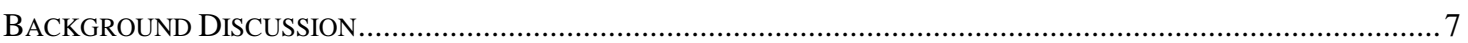

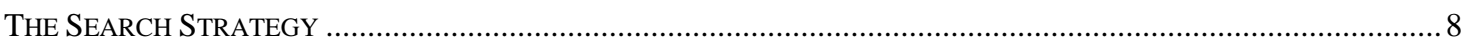

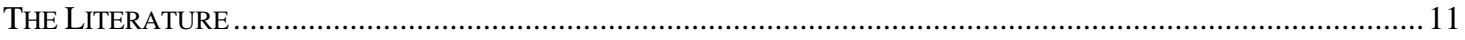

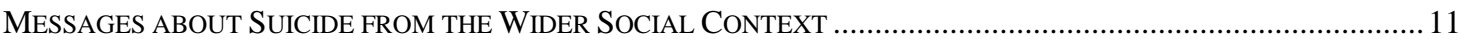

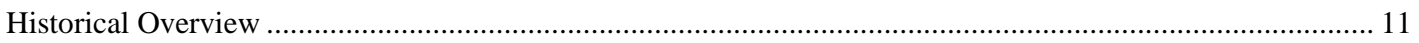

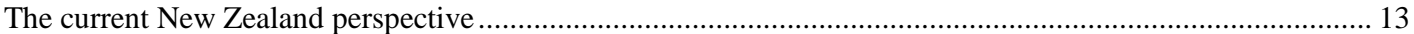

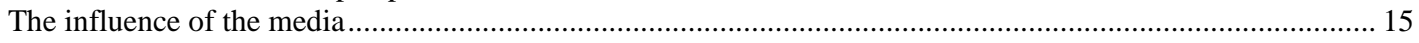

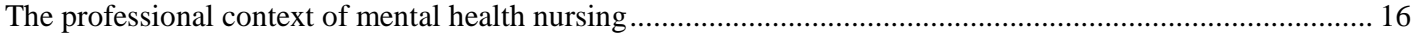

Role definition and the future and direction of mental health nursing ...................................................... 17

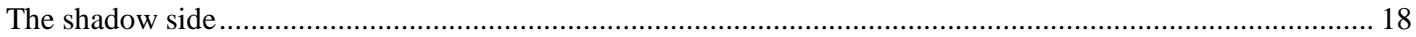

PHILOSOPHY OF CARE AND MODELS OF CARE ...................................................................................... 20

MESSAges ABOUT THE EFFECTS OF THEIR WORK WITH PEOPLE EXPERIENCING SUICIDAL IDEATION ON MENTAL

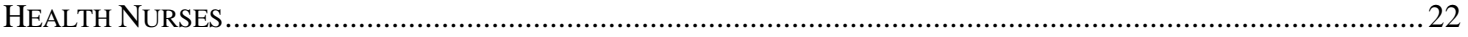

CHAPTER THREE: METHODOLOGY AND RESEARCH DESIGN.................................................. 25

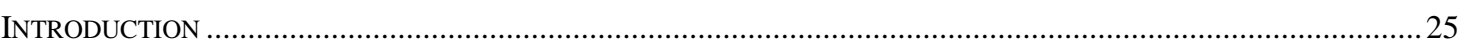

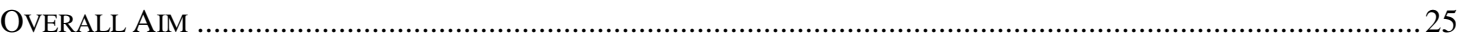

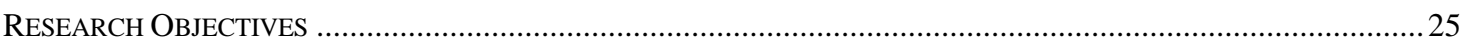

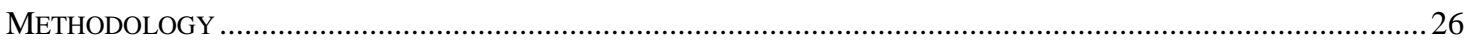

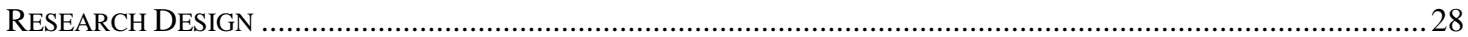

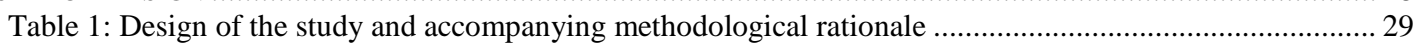

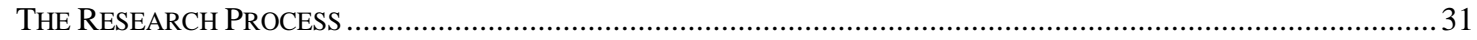

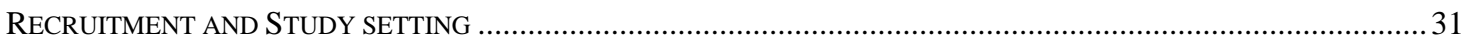

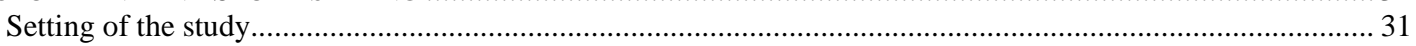

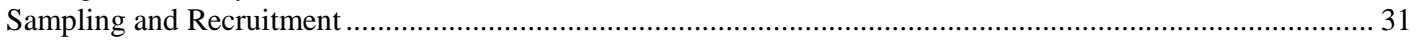

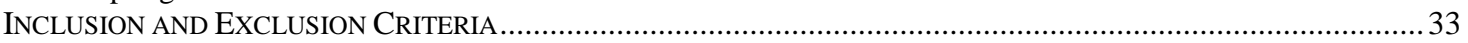




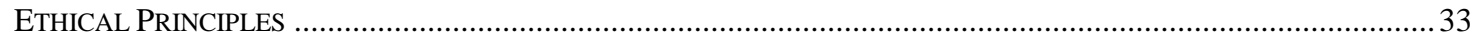

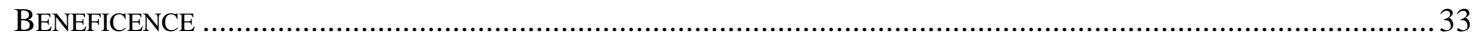

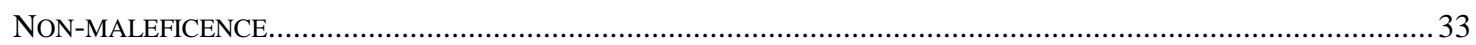

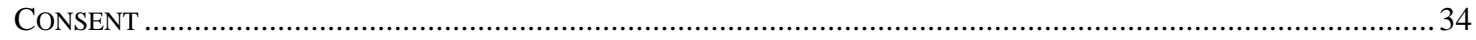

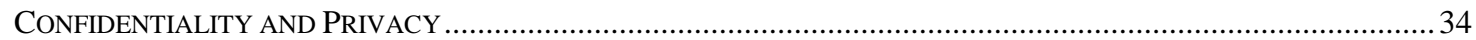

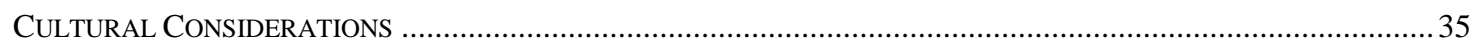

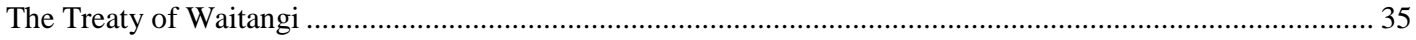

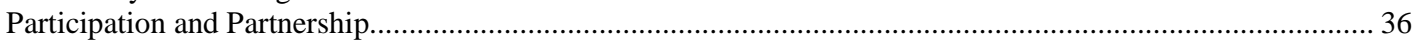

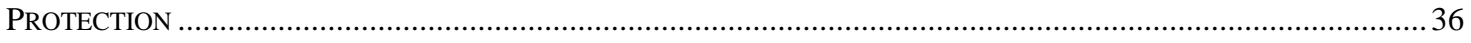

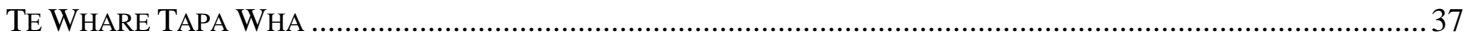

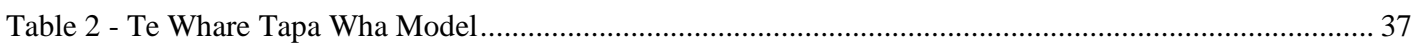

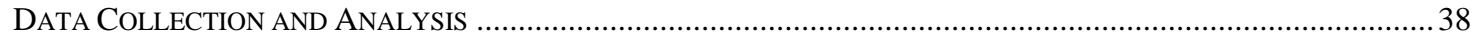

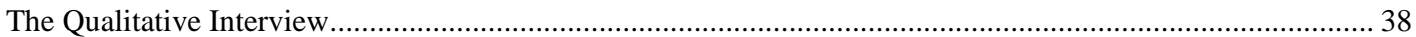

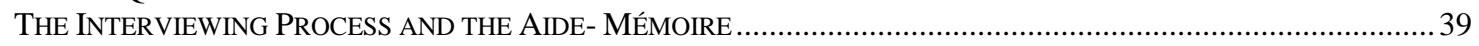

DATA STORAGE

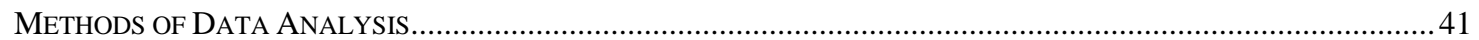

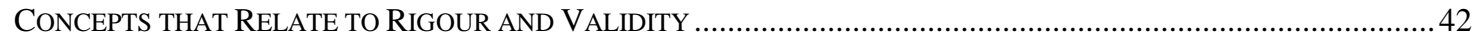

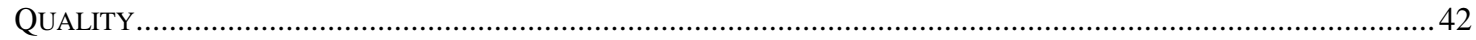

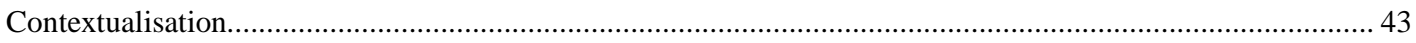

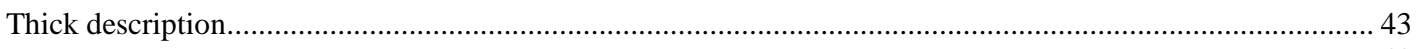

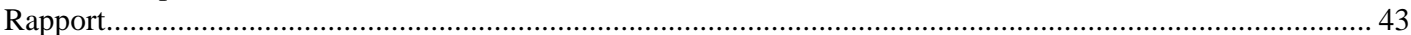

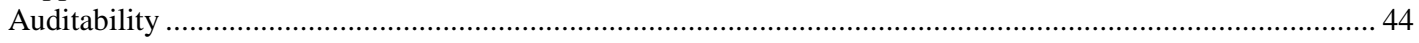

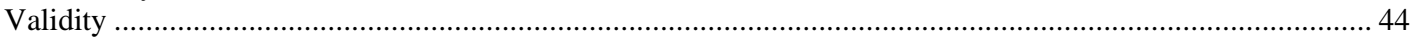

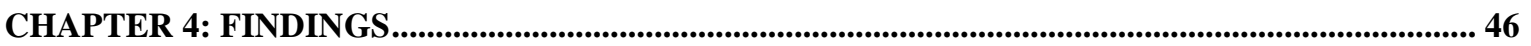

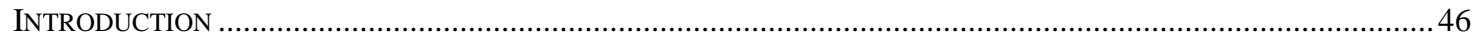

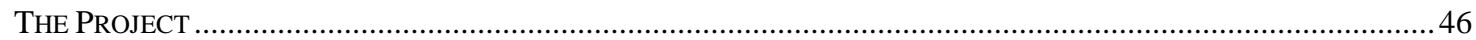

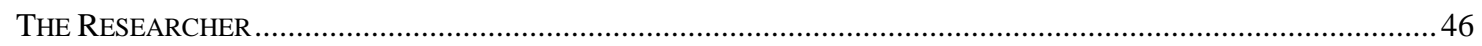

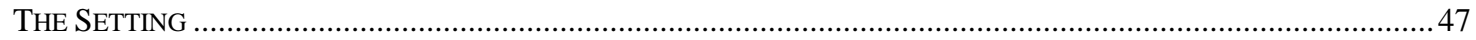

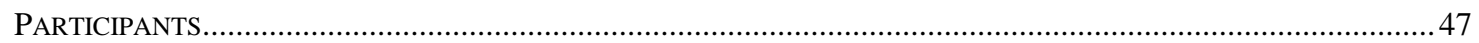

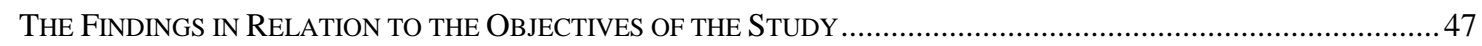

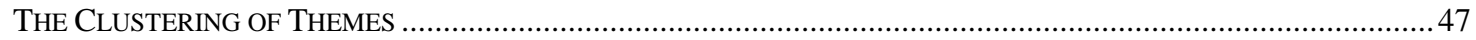

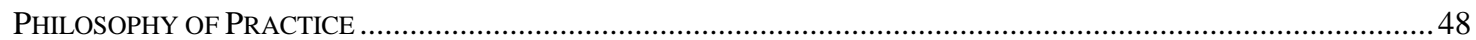

Philosophy of Practice in Relation to Working With People ExPeriencing Suicidal IdeAtion ..........48

PHILOSOPHY OF PRACTICE IN RELATION TO WORKING WITH COLLEAGUES.................................................51

PhILOSOPHY OF PRACTICE IN RELATION TO ORGANISATIONAL AND PROFESSIONAL EXPECTATIONS...................52

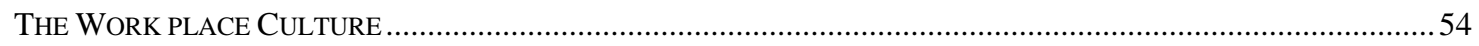

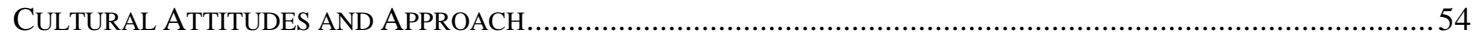

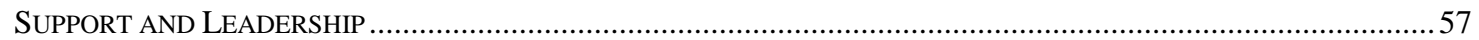

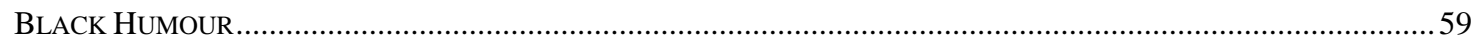

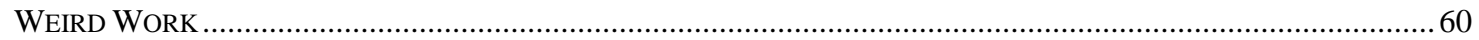

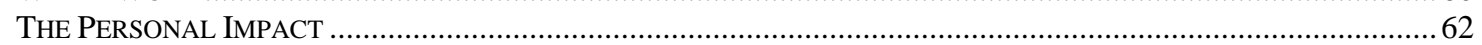

THE SPIRITUAL DOMAIN - TAHA WAIRUA- THE CAPACITY FOR FAITH AND WIDER COMMUNION .......................62

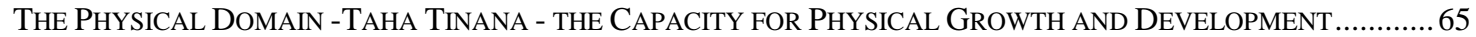

The Mental/Emotional Domain - TAHA Hinengaro- THE CAPACITY TO THINK AND TO FeEl ........................67

The Whanau/Social domain- TAHA WhanaU - THE CAPACITY to Belong to Share AND to Care...........69

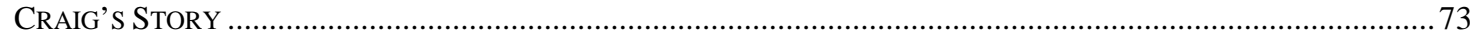

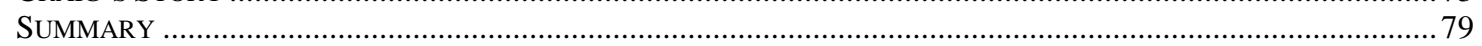

CHAPTER 5: DISCUSSION, IMPLICATIONS \& CONCLUSION.......................................................... 80

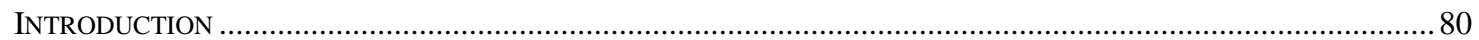

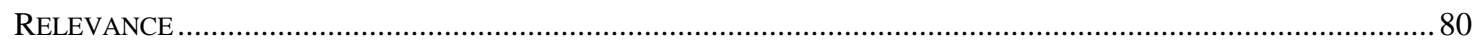

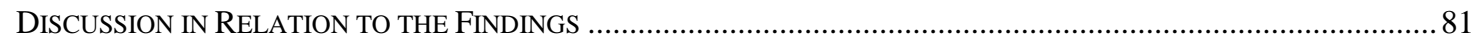

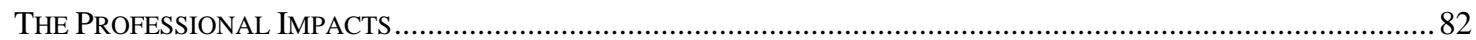

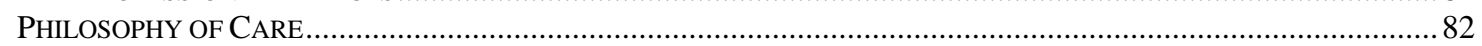

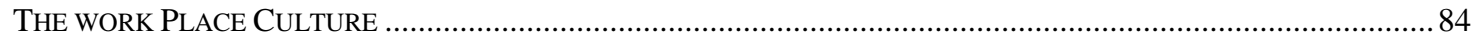

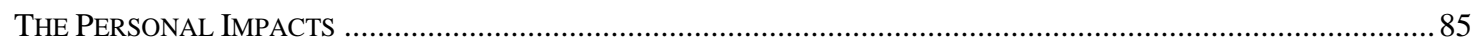

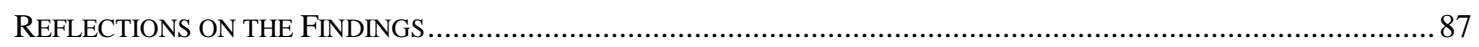

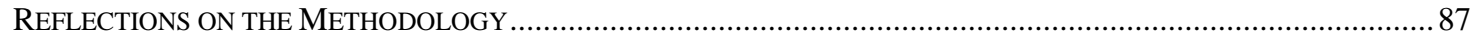

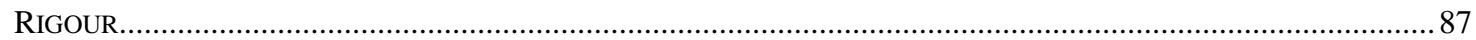

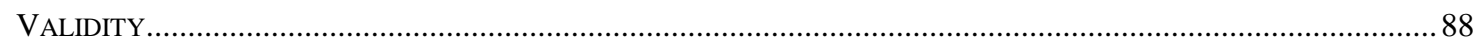




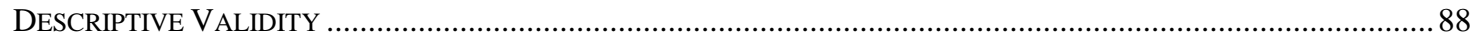

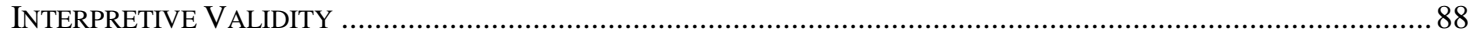

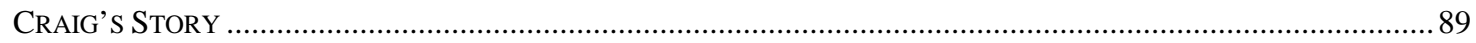

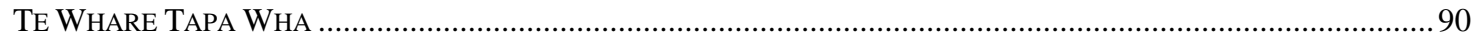

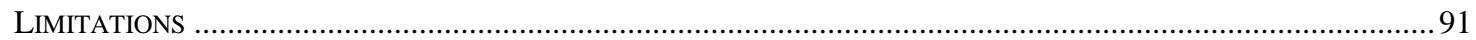

IMPLICATIONS AND RECOMMENDATIONS ARISING FROM THE DISCUSSION ............................................... 91

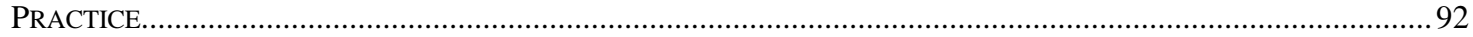

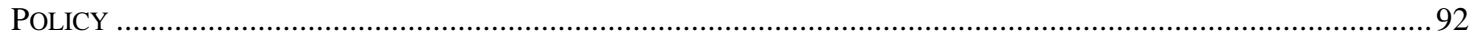

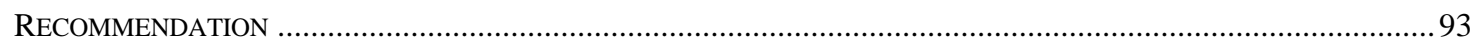

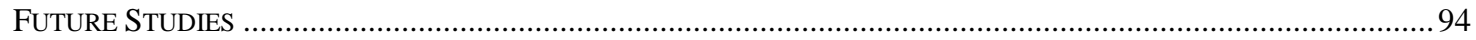

REFLECTIONS ON THE LEARNING GAINED AS A RESULT OF UNDERTAKING THIS STUDY ................................95

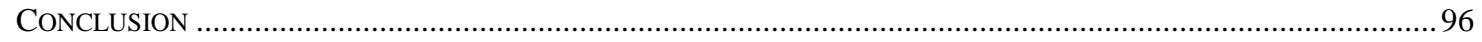

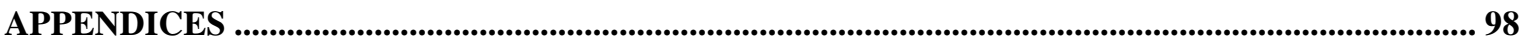

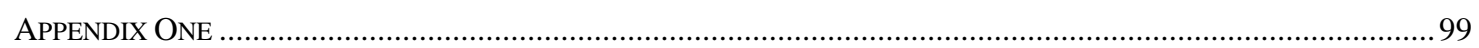

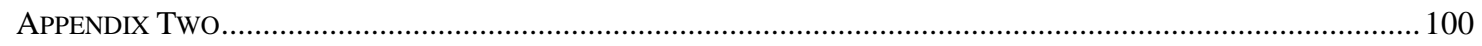

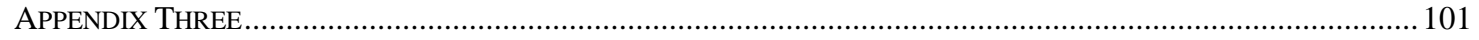

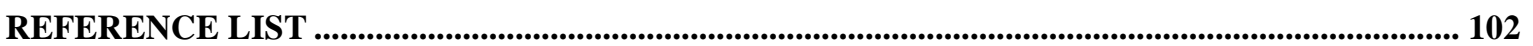




\section{List of Tables}

Table 1: Design of the study and accompanying methodological rationale . 29

Table 2 - Te Whare Tapa Wha Model 


\section{Glossary}

The following terms are unique to New Zealand and are used in this thesis.

Whanau

District Health Board

Treaty of Waitangi
Maori word meaning family or extended family.

The twenty one New Zealand District Health Boards (DHBs) are responsible for providing, or funding the provision of, health and disability services in their district in New Zealand.

The Treaty of Waitangi was signed in 1840 by representatives of the British Crown and by various Maori Chiefs from around New Zealand. It is regarded now by many as the founding document New Zealand as a nation. The treaty established a British Governor and recognised Maori ownership of their lands and other property and gave Maori the rights of British subjects. Maori and English versions of the treaty differ and today understandings about what was agreed are still being debated. In 1975 a Tribunal was set up to address many grievances that were a result of the Treaty being largely ignored by various New Zealand governments since 1840 . 


\section{Chapter One: Introduction}

\section{The Research Question}

What impact does working with people experiencing suicidal ideation have on the lives of mental health nurses working in an acute mental health setting?

\section{Research Aim, Objectives and Focus}

The aim of this research is to provide a description of the impact that working with people experiencing suicidal ideation has on the personal and professional lives of mental health nurses who work in acute mental health settings in New Zealand. Three key research objectives were identified in relation to this. These are as follows:

- To gather information from mental health nurses about their experiences of working with people experiencing suicidal ideation in relation to their philosophy of practice and that of their colleagues;

- To explore nurses' experience of working with their work place culture and organizational and professional expectations in relation to their way of working with people experiencing suicidal ideation;

- To discover what influence and impact this work has on the physical, spiritual, mental, emotional, social and whanau (family) aspects of their lives.

\section{Title of Thesis}

The title of this thesis emerged from the key findings of the research and acknowledges that, for the nurses who participated in the study, there were professional and personal impacts that resulted from their work with people experiencing suicidal ideation and that some of these impacts were profound and, for some, life changing.

\section{Relevance}

This study has wide relevance because according to the World Health Organisation, someone, somewhere, in the world commits suicide every 39 seconds (Wikipedia, 2008). In fact about one million people a year die by suicide, which is more than the total murdered or killed by war. An estimated 10 to 20 million non-fatal attempts to suicide were also made during this time.

This study has special relevance in New Zealand because the country's rate of suicide is among one of the highest in the western world. This problem is regarded by the 
government as "a serious health and social issue which represents a significant loss to our society" (Associate Minister of Health, 2006). Approximately five hundred New Zealanders die by suicide every year and more than five times that number will be hospitalized as a result of a suicide attempt (Associate Minister of Health).

When considering these figures it is not surprising that the management of a suicidal crisis as a result of a suicide attempt is the most frequently encountered mental health emergency and that this type of emergency is acknowledged as amongst the most difficult and frightening challenges faced by mental health professionals (Roberts, 2000).

This study is directly relevant to nursing because, in New Zealand, it is most likely that it will be a nurse who carries out the first assessment of a person following a suicide attempt. They will also be required to decide on the level of follow up needed and will often continue to engage with, and coordinate much of the care for, these people that are often still highly at risk of completing suicide (Mental Health Commission, 2001).

The findings from this study offer some insights into the impact that this work has on the lives of some of these nurses and so has relevance in what it can offer in terms of adding to existing nursing knowledge to do with nursing people experiencing suicidal ideation. The findings from this may also hold some relevance for other professionals and groups engaged in working with people experiencing suicidal ideation.

\section{The Significance of the Study}

This study is small. Five mental health nurses are not enough to generate sufficient data to fully answer the research question. However, the information provided by the five participants does reveal their experiences and insights. This goes to creating some understanding into the impact that working with people who are experiencing suicidal ideation can have on mental health nurses. It is important also that further research and investigation in to this be undertaken.

\section{Contribution to Nursing Knowledge}

It is hoped that the knowledge uncovered in this project will be useful to other mental health nurses who work in similar settings to those who participated in the project. It may assist them to recognise and assess the impact of their work on themselves, and on others who work alongside them, and help them to reflect on their own practice and the 
impact of this on their colleagues. This knowledge would also be useful as a basis for a critical, reflective, discussion in teams that have a similar makeup to those in which the participants practiced.

The findings may also be useful to nurses, or others interested in undertaking further research, as this project could be regarded as the results of a small explorative pilot study that could lead into a number of studies into any of the aspects uncovered here.

\section{Personal Background}

I am a Registered Comprehensive Nurse with seventeen years experience in mental health nursing. I spent my first four years of practice working in a large psychiatric institution before going on to practice in a community based crisis mental health team for the next ten years. I have had experience in a number of statutory, educational and managerial roles and continued to further my education during this time.

My personal and professional beliefs are informed by the phenomenological tradition of western philosophy. In the helping sense, this is probably best explained by Rogers (1986) who suggested that rather than trying to change a person's reality, the task of helping is to respect and understand his view of himself in the world. In practice, it has sometimes felt as though working from my philosophy of practice challenges rather than supports me and it is the sense of dissonance that comes from this experience that has made me curious about how other nurses experience their work with people experiencing suicidal ideation.

\section{Background}

My interest in this project was prompted by reflections on my own practice, what I was learning as a result of further education and on realising the complexity of interpersonal dynamics as I observed them unfolding in a service that provided acute mental health care for people experiencing suicidal ideation. My interest was further provoked by many conversations held with other nurses who spoke freely about their views on this work. As a result of these reflections, it occurred to me that working with people experiencing suicidal ideation presents a more complex challenge for nurses than that posed by their work with people who were living with other symptoms of mental distress. 


\section{The Wider Context}

In the previous section I provided background information about what provoked me to undertake a research project in this area of interest. In this next section I present some further information in order to give a wider understanding of the background context of the study.

\section{The professional context}

Nurses who work with people experiencing suicidal ideation practice in uncertain times. This uncertainty is perhaps best reflected in the inability of the profession, (if it is one profession), to reach consensus about what 'we' should be called and there are many different philosophical interpretations of what constitutes the title of a mental health nurse and/or a psychiatric nurse (Barker, 1999; Peplau, 1988; Watson, 2002).

Contemporary mental health nursing also exists within a context of changing sociopolitical views of mental health issues and factors such as the growing consumer movement have challenged the traditional roles and relationships that used to provide the philosophical imperatives from which mental health nurses practiced (Barker, 1999; Crowe, 1997; Watson, 2002).

In New Zealand, the change from institutional care to community care has added to uncertainties faced by nurses. This has been attributed to the loss of skilled nurses from the sector, the need for different skills and knowledge, inconsistencies in nursing leadership, poor staff retention, inconsistent education standards and there being insufficient and inconsistent clinical supervision available (Ministry of Health, 2006).

\section{The Study}

This project sought to establish new knowledge by providing insights into the experiences of mental health nurses who work with people who are experiencing suicidal ideation. Explorative studies like this are best fitted to a qualitative descriptive approach. This project follows the tenets of Sandelowski's (2000) interpretation of this methodology.

\section{Limitations in Research Foci}

I chose to limit the focus of the project and only investigated nurses' experiences of this work. This choice was made on the grounds of ensuring that the study remained within the scope of a small masters' project. 
The study was also limited to collecting information that was relevant to the research aims. This limitation also ensured that the project stayed within the scope of a small masters' project and that the project was able to retain its focus of enquiry and keep true to its methodological underpinnings.

\section{Definitions}

The following definitions have been included for the purpose of clarification - Suicidal ideation is defined by Roberts as "Any self reported thoughts of engaging in suicidal behaviours" (2000, p.526). It is more simply defined in the Ministry of Health's Suicide Prevention Strategy as "which refers to thoughts of suicide". The strategy also includes a definition for suicide that, "refers to the act of intentionally killing ones self" (Associate Minister of Health, 2006 p.3). Further, in New Zealand classification of death as suicide is based upon a coronial verdict.

Other useful definitions in the Ministry's strategy document are the one for deliberate self harm; "behaviours that may or may not result in serious injury but are not intentionally fatal" and attempted suicide, which "covers a range of actions where people make attempts at suicide which are not fatal" (Associate Minister of Health, 2006 p.3).

\section{Language}

These terms have been identified for the purposes of clarity.

The term person is used throughout the study to identify those who have used mental health services.

The term participant is used to differentiate between those nurses who took part in the study and other nurses are referred to but who did not take place in the study.

The term mental health nurse was chosen to describe those nurses who have specialised in mental health nursing practice.

\section{Structure of this Thesis}

The opening chapter introduces the study by explaining the aims and rationale for the research and providing some of background context in which it is located. The following chapter presents an analysis of the literature and discusses how this led to the formulation of the study design. Chapter Three discusses the rationale for the 
methodological approach and the processes used in the study's investigative phases. The findings from the study are presented in chapter Four. Chapter Five goes on to discuss these and the implications that arise as a result of these. I make some recommendations in terms of policy, practice and future studies and the chapter closes with reflections on the project in terms of its design, methodology and limitations. 


\section{Chapter Two: Literature Review}

\section{Introduction}

The purpose of undertaking a literature review is so that a researcher can learn about what is already known about the topic they are interested in. They can then establish what other questions to do with the topic need to be answered and also work out how the knowledge gained from answering the question will fit into the existing body of literature. A literature review also assists a researcher to narrow down the scope of the topic selected to a manageable size and helps when it comes to choosing the method and study design best suited to answer the question (Minichiello, Sullivan, Greenwood \& Axford 1999; Roberts \& Taylor, 2002; Sandelowski, 2000).

In the following chapter I give some background into the processes that were undertaken to explore the literature and the rationales that formed the basis for these. I then go on to discuss the literature as it relates to the context in which this study took place. I conclude the chapter with a brief discussion about the relevance of this literature in relation to the aims and objectives of the study.

\section{Background Discussion}

In this next section I explain the rationale for the selection processes used in relation to the literature that is discussed in this study. The explanation begins with identifying the key assumptions that informed the decision making processes in regard to the literature review and its place in the study design then moves on to explain the search strategies that were employed to elicit the literature in relation to the method and research objectives of the study.

This study sought to explore and describe the impact that working with people experiencing suicidal ideation has on mental health nurses by asking five mental health nurses about their experiences of doing this work. Studies that explore subjects such as these are usually placed into the paradigm of qualitative research. One of the key assumptions of qualitative research is that a phenomenon or event cannot be understood in isolation and that people's behaviour is affected by their environment and experiences so therefore, a specific behaviour or attitude cannot be investigated without taking into account all things which will affect the person's perceptions and actions (Minichiello et al. 1999). 
One of the reasons Sandelowski gives for presenting her work on fundamental qualitative description was that she shared the concerns of Thorne, Kirkham and MacDonald -Emes (as cited in Sandelowski, 2000) who proposed that that many researchers were appeared to be performing "methodological acrobats"(p. 335) in their seeking of epistemological credibility. Sandelowski proposed that researchers have felt obliged, because of their perceptions that a methodological hierarchy of importance exists, to designate their work as phenomenological, narrative, grounded theory or ethnography, when their work contains only hints or hues of the method they have claimed to follow. Sandelowski suggests instead that researchers who wish to provide a straight description of an event or phenomenon should do so and, unashamedly, claim fundamental qualitative description as their method. If they then choose to design their studies using overtones from other methods, they should simply describe what these overtones were.

Specific to the naturalistic tenets described by Lincoln and Guba is that researchers "endeavour to describe the phenomenon or event in its natural state, or as it is, to the extent that this is possible in a research enterprise" (as cited in Sandelowski, 2000, p.335). That is, that there is no prior commitment by the researcher to any theoretical view of a target phenomenon and techniques should be used that allow the target phenomenon to present its self, as if it were not under study (Sandelowski, 2000).

\section{The Search Strategy}

Roberts and Taylor (2002) define literature as "the total body of writing that deals with the topic being researched" (p. 534). At the beginning of this project I had some ideas about what I wanted to achieve in terms of learning but was still unclear about how to manage the contextual complexities that needed to be considered when undertaking a qualitative study in a practice setting. In order to try and make sense of these, I conducted an informal search using the key word 'suicide' on the 'Google' search engine. This achieved 75,750,000 strikes in fourteen seconds. When I changed the words to suicidal ideation, the number of strikes still reached 35,500. The sheer scale of literature available on the topic in terms of the wider context in which mental health nurses practice was overwhelming when considering this against a small scale study. Encouragingly, after changing the search engine to 'Google Scholar', the number of strikes reduced to 12,200 . Scanning through some of the articles available on Google 
Scholar helped me to become more focused on the area of study I wanted to pursue. I then moved to conducting a formal search via the data bases of CINAHL, Medline, and PsycINFO.

Initial searches using the words mental health nurse, psychiatric, suicidal ideation and impact uncovered articles on research carried out into the effects of completed suicide on clinicians (Alexander, Klein, Gray, Dewar \& Eagles, 2000; Deardon, 2004), research articles into the part that constant observations play in the care of people experiencing suicidal ideation (Fletcher, 1999; Baker \& Cutcliffe 1999) and articles that explored stress as it effects all nurses and specifically mental health nurses (Clegg, 2001; Pryjmachuk \& Richards, 2007; Stordeaur, D’Hoore,\& Vandenberghe, 2001. Another article uncovered in one of the later searches identified resources needed to reduce stress in mental health nursing.(van den Tooren, 2008). Other articles looked at vicarious trauma in relation to mental health nursing (Clark \& Gioro, 1998; Happell, Pinikahana \& Martin, 2003). Some of these articles provided information that was relevant to the wider context of the subject; however the searches undertaken did not uncover any evidence of any research that had explored the impacts that working with people experiencing suicidal ideation has on mental health nurses in terms of their whole lives.

Interestingly, while undertaking this review, I became aware that I had begun to reflect on some of what I was reading and realised that I was being drawn toward interpreting some of what I had observed in practice through the constructs that were proposed in the literature. At this point I stopped and reflected on what it was that I wanted to achieve. As a result I became clear that my interest lay in wanting to describe the impacts that working with people experiencing suicidal ideation has on the whole lives of mental health nurses. It was also important to me that the end result was a representation of the collective voice of nurses in practice and that it should remain as free as possible from any presuppositions that could occur as a result of my unconsciously including language and terms that relate to a particular theoretical perspective during the interview process.

Gaining a clear picture of what I wanted to explore led me to select the method of fundamental description, which is championed by its proponent as "the method of 
choice when straight description of phenomenon is desired" Sandelowski (2000, p.337). This method will be more fully discussed in Chapter Three.

After reflecting on the tenets of qualitative research and on Sandelowski's (2000) ideas on qualitative description, I decided to design the study so that the key findings from the collected data were established prior to any further review of the literature. My rationale for this was that the literature review to date had already established that there was little or no evidence of prior research having been carried out into this specific area of interest.

Another advantage of carrying out the second literature review after the findings had been established and prior to the final discussion in the study, was that the discussion chapter was able to offer more in terms of the relevance of the study in relation to practice, policy and future research initiatives.

Taking a second look at the literature also meant that the chance of me, as the researcher, unconsciously leading participants toward a particular construct was lessened. This idea is consistent with principles usually applied when undertaking a research project informed by the method of grounded theory (Roberts \& Taylor, 2002). Sandelowski (1986) describes this as "bracketing", which means that the researcher attempts to minimize their own preconceptions and biases so as to allow the data to speak for itself. This study departs from other tenets of grounded theory as the intention of this study was to describe the phenomenon, rather than to generate data for the purpose of building on or changing existing theory.

After the findings of the study were revealed, a further search of the literature was undertaken using the data bases of CINAHL, Medline, and PsycINFO. For this search I used combinations of the following key words: psychiatric mental health nurses, spirituality, stress, impact, suicidal ideation, acute care, humour, models of care and philosophy. The consistent words used in the searches were suicidal-ideation, mentalhealth, and psychiatric nursing. Articles were selected by reading the abstracts of the articles selected by the search engines for their relevance to the study. These articles were then sorted into categories of systematic reviews, individual research papers (including literature reviews), opinion papers, and other sources. The search was limited to a ten year period from 1998 to 2008, the articles were required to be written 
in English and they had to have an abstract. This time-limit was sometimes extended if any work was particularly pertinent, or if the literature referred to was considered to be a seminal work in the area under discussion. The reference lists from selected articles provided a rich source of information and often indicated who the key proponents were in the areas of interest. Offerings from colleagues and allied health professionals also proved to be valuable sources of information and were especially relevant to the current culture and context in which the study took place.

In all, 3,924 articles were retrieved. Two hundred and six articles related to workplace stress and vicarious trauma, 1,726 articles related to philosophy of practice and 1,987 to models of care. Five articles related to spirituality in mental health nursing and 475 related to humour in nursing.

\section{The Literature}

In this next section I discuss the literature in relation to context in which this study took place and in relation to the messages that are out there about the effects on mental health nurses as a result of their work with people experiencing suicidal ideation.

I have divided this section of the chapter into four subsections; messages about suicide from the wider social context, the professional context of mental health nursing, philosophies and models of care and messages about the effects of their work on mental health nurses.

\section{Messages about Suicide from the Wider Social Context}

I start this section by providing a brief history and cultural perspective of how the subject of suicide has been understood by different populations in the world. I then discuss the literature as it applies to the current government policies in New Zealand that provide direction for the care of people experiencing suicidal ideation. I also discuss the influence of the media in the portrayal of suicide in order to provide a wider context from which to understand the practice worlds of the participants.

\section{Historical Overview}

Over the years, constructs about understanding suicide throughout the world have been largely influenced by cultural views on existential themes such as the meaning of life, honour and religion. Religious beliefs have proven to be the most influential, however these were often moderated by financial imperatives (Wikipedia, 2008). 
No one knows who the first person was to kill themselves but we do know that early records tell of many historical figures who chose to end their lives rather than to face dishonour or in order to uphold a religious or philosophical principle. Examples of these include Hannibal who took poison to avoid capture and Socrates who chose to end his life rather than renounce his beliefs. Gladiators were also known to have stabbed themselves to avoid taking the lives of others (Jamison, 2000).

The Bible tells that no sanctions were attached to the suicides described in the Old Testament or to the suicide of Judas Iscariot described in the New Testament after his betrayal of Christ. However, it seems that Christian attitudes hardened later (Jamison, 2000).

In England, during the eighteen hundreds, suicide was regarded as a crime against God and the King and so was regarded as doubly offensive. The crime was punished doubly, as not only was the person's corpse denied burial in consecrated ground, their worldly goods were confiscated too. This changed only after Edwardian juries became reluctant to punish the families of suicide victims and began to find that the person had died as a result of their being insane. Interestingly, this transformation from 'criminalizing' to 'insanitising' happened long before the medical specialty of psychiatry existed (Hillman, 1997).

The act of suicide was viewed as a crime in England until 1961 and in Ireland until 1993 (Jamison, 2000).

The body of a suicide was often dishonoured in many countries. The French dragged them through the streets then hung them face down from the gallows. In other countries, stakes were driven through the hearts of suicides and their bodies were buried at cross roads with the idea the spirit would find it hard to find its way home. Bodies were often not washed and were buried without funeral services (Jamison, 2000). Jewish tradition forbade funeral rites and burials were limited to a section outside the graveyard. This tradition was also common in Christian rituals, where suicides were buried with criminals and those who were not baptized. Later, compassion was extended to Jewish families and they were able to take comfort for their loss from other Jewish people however, no honour was allowed to be given to the victim (Jamison). 
In India, although the Hindu population regarded suicide as a dishonourable act, they also accepted the practice of suttee, the act of a widow who, with or without encouragement from family, throws herself onto her husband's funeral pyre so that she will not have to be kept by the family (Wikipedia, 2008).

Different forms of suicide have long been regarded as part of the Japanese culture. In the era of the Samurai, the Japanese people came to regard the practice of seppuku as a means by which to restore honour after failure and, years later, kamikaze pilots used their planes as bombs and were revered as martyrs who had died for their emperor and country. Even today, many Japanese continue to perform seppuku, a ritualistic form of suicide, as a means of face saving should they fail in business or in some other endeavour (Jamison, 2000).

In the later twentieth century, self immolation became almost fashionable as a form of protest or as a way of making a political statement in countries such as Germany, India and the United States. Today, the act of suicide bombing is viewed by the followers of Islam as an acceptable act in a holy war (Wikipedia, 2008). Interestingly, Islamic law still finds suicide a greater crime than that of homicide (Jamison, 2000).

The ethics of assisted suicide and euthanasia also continues to be hotly debated issue in most countries while in the same world, the Inuit people of the far north of the American continent are still accepting that the weak in their community will take themselves out into the snow to die as a means to protect the resources of their family. Sadly, it is now not only their weak (Jamison, 2000).

As stated previously, suicide remains one of the biggest causes of death in the world and, despite research into its cause, the best modern medicines and the best efforts of many, the figures continue to rise.

\section{The current New Zealand perspective}

The predominant view of modern western medicine is that suicide is a mental health concern associated with factors such as coping with depression or inescapable suffering or fear as a result of a major mental (Wikipedia, 2000). Despite the view of suicide as a mental health concern, many other reasons are given for suicide by those who leave notes to explain their reasons for ending their life. Unrequited love, grief, escape from punishment or abuse, guilt or shame, catastrophic injury, financial loss, fear of aging, 
unresolved sexual issues, a belief that life has no inherent value and even curiosity about post life experiences have all been cited (Hillman, 1997; Jamison, 2000).

The view of suicide as a mental health concern is reflected in the strategy that the Ministry of Health has undertaken in its bid to reduce the countries high suicide rates (Associate Minister of Health, 2006). This strategy was developed eight years after the release of the New Zealand Youth Suicide Prevention Strategy (Ministry of Youth Affairs, 1998), which was developed in response to the country's alarmingly high youth suicide rates and had been successful in terms of reducing the male suicide rates for this age group.

Although the strategy acknowledges Collings and Beautrais (2005) and their social explanations for suicide, most of the document is presented in clinical language. Interestingly, a model presented in the document identifies the social issues that can contribute to suicidal ideation then, with the addition of an arrow from one a box to another, reinterprets these factors into psychiatric diagnoses.

The strategy outlines seven goals and two appear to have implications for mental health nurses. These are goals three and four which are to improve the care of people who are experiencing mental disorder associated with suicidal behaviour and; to improve the care of people who make non fatal suicide attempts. The actions identified as ways of meeting these goals are vague and non specific in regard to whom they apply. They include "improving the capability of health professionals and other key personnel to respond appropriately to people experiencing symptoms of mental disorders" and "improving methods of treatment and management, after care and support" (Associate Minister of Health, 2006, pp 24, 25). The vagueness of the New Zealand Suicide Prevention Strategy is in keeping with other government policy documents and this could be because such documents are intended to provide direction rather than be prescriptive in terms of specific interventions.

A document with more direct relevance for mental health nursing is "Guidelines for Primary Health Providers: Detection and Management of Young People at Risk of Suicide" (Ministry of Youth Affairs, 1999). This document was developed in response to the New Zealand Youth Suicide Prevention Strategy (Ministry of Youth Affairs, 1998) as a resource for use by general practitioners and also as a resource for continuing 
medical and nursing training and for general practice or nursing training. Although it is medically focused, it provides a comprehensive overview of information and offers real intervention strategies for those who work with young people experiencing suicidal ideation. This document also acknowledges the impact that the suicide of a young person can have on care providers. It gives advice to readers about how to talk with the media about suicide.

\section{The influence of the media}

The influence of the media in the portrayal of suicide in the community has been thoroughly researched both overseas and in New Zealand and, although there is debate about the rights of the press versus the protection of the vulnerable, there is agreement that media portrayal has a large influence on how suicide is viewed by the public, both in New Zealand and in the rest of the world (Tully \& Elsaka, 2004).

In 1999, the Ministry of Health developed a set of guidelines for media reporting of suicide (Ministry of Health, 1999). However, a study that aimed to scope the awareness of media professionals about the guidelines found them to have been largely ignored due to their belief that the guidelines were too restrictive (Tully \& Elsaka, 2004).

The role of the press in relation to the way New Zealanders perceive suicide is scrutinised by The Mental Health Foundation of New Zealand who fund a 'watchdog' organisation called the Suicide Prevention Information New Zealand (SPINZ). SPINZ monitors the reporting of suicide by scanning the country's newspapers daily and keeps copies of any articles that refer directly or indirectly to the subject of suicide. They often speak out against publications they believe are responsible for sensationalising suicide. While SPINZ is most commonly concerned about press coverage that could lead to what is termed as 'the contagion effect' (Goldney, 2001; Hawthorne \& Williams, 2001; Sudak \& Sudak, 2005), they also hold other articles in their files that provide a commentary on the different attitudes toward suicide held by members of the general public. On a trip to their offices in Auckland I uncovered a number of articles that reported on aspects on the subject of suicide, such as the impact that suicide has on family members (Richardson, 2005) and about a mother's request to have suicide kept as a private family matter (Hapete, 2005). 
While most of the articles I discovered in the SPINZ files were neutral in terms of any specific messages given in regard to mental health nurses and their work with people experiencing suicidal ideation, one article was not. It reported on a family's intention to sue a District Health Board (DHB) for negligence following an incident during which their son had left an inpatient unit and had taken his life. Although the report spoke of systemic failures, it also claimed that individual nursing staff had failed to keep the man safe. The article also took the opportunity to raise attention to other failures on behalf of nursing staff by reminding its readers of previous incidents that had occurred around the country with similarly tragic outcomes (Richardson, 2005).

Health professionals have also been known to use the opportunities afforded to them by the press in order to respond to similar criticisms. An example of this was when the head of a mental health service defended the actions of his crisis assessment team who reportedly had assessed a person and who later in the same night set fire to themselves (Langdon, 2000).

Accessing the press can also be a useful way for individuals to pass on their own messages about suicide, such as when Deborah Coddington, a then Member of Parliament, wrote an article that appeared in the Herald on Sunday. In it she told of a chance meeting with a friend who had a child die by suicide. As well as describing her own discomfort about not knowing what to say to someone who had lost someone under such tragic circumstances, she used the article to bring to attention a point made by an Auckland University psychologist who "suggested that if stress and depression weren't classified as mental illnesses then young people might be more willing to seek help without being labelled as 'nutters' (Coddington, 2005, p. 35).

\section{The professional context of mental health nursing}

In this section I discuss the literature as it relates to the professional context of mental health nursing and working with people experiencing suicidal ideation. I begin by discussing the literature that reflects the current issues facing mental health nursing as a profession and then discuss some of the literature as it pertains more specifically to working with people experiencing suicidal ideation. 
Role definition and the future and direction of mental health nursing

Defining the role of nursing has been discussed since the days of Florence Nightingale who complained in 1860 that "no man, not even a doctor, ever gives any other definition of what a nurse should be than this, - devoted and obedient" (as cited in Lauder, Forest \& Masters, 2006). Although defining the role of the mental health nurse remains as a prominent concern for many in the profession, defining the role is seen as a secondary concern by Lauder et al. who believe that nurses should focus on what mental health nurses are needed for.

The literature shows that world-wide concern is being expressed by mental health nurses about the current state of the profession in relation to its purpose and direction. Various writers and researchers have offered reasons for why this is so and most agree that internal and external socio-political forces are the main influences currently guiding the direction of the profession. Most suggest that until some of these issues are resolved, the future of the profession remains uncertain (Barker, 1999; Ministry of Health, 2006; O'Brien, Thom \& Hughes, 2007). It seems that most agree extraneous social and political influences such as the consumer movement, the shift from institutionally based care to community care and financial imperatives have all contributed to this situation (Barker, Crowe, O’Malley \& Gordon, 2001). In most papers, the authors also refer to the influence of the psychiatric paradigm or the medical model on the mental health nursing profession and question whether past allegiances are still of value to nursing, and to the people mental health nurses profess to serve (Barker, Crowe, 2000; Crowe, O’Malley \& Gordon; O’Brien, Thom \& Hughes).

Marie Crowe, a scholar who features prominently in New Zealand mental health nursing literature, spoke of this in a paper published in 2000 saying:

If mental health nursing practice is a patient centred partnership, as many of our nursing standards suggest, then nursing's focus should be on the patients' experience rather than the psychiatric diagnosis with which the experience is attributed. Mental Health nurses need to turn to service users to learn how to best help. (p 583)

This paper was critically reviewed in 2006 by Phil Barker, another prominent scholar who, although hails from Scotland, has taught extensively in New Zealand and has left 
his mark here through the adoption of the Tidal Model ( Barker, 2001) in various mental health settings in New Zealand (Cook, Phillips \& Sadler, 2005). In his review he reflects that Crowe's paper is more relevant and more provocative today than it was when it was originally published. He claims that the current trend in western countries is for more and more problems involving human behaviour to become understood within the framework of psychiatric diagnosis and that mental health nurses tacitly support this by their use of the language dominant in this discourse in their communications with others. He observes that there seems little willingness on the side of nurses to further develop "the language of interpersonal caring that once held the promise of a distinctive nursing voice" (Barker, 2006, p. 133). Interestingly, he finishes his review by suggesting that "Crowe's status as New Zealand academic may provide a contextual referent, signalling a way forward out of this impasse" and that New Zealand, with its record of recognizing the voice of its indigenous people might lead the way in this" (p 133).

The view that mental health nursing has become reliant on the medical constructions inherent in psychiatric diagnosis is also supported by authors Bishop and Ford-Bruins (2003) who add that, despite having the skills to conduct assessments; nurses' contributions are often minimized within multidisciplinary teams.

The shadow side

In this subsection I discuss the literature in relation to the darker side of mental health nursing and its history in relation to oppressive practices. The section also includes discussion about the personal challenges faced by mental health nurses as a result of their work in general and in relation to people experiencing suicidal ideation.

I have borrowed the term 'the shadow side' from Peter Watson ( 2002) who believes that many people who are drawn to work in the mental health profession do so because of an unmet need in them, which can interfere with the way in which they engage with people. Watson refers openly to past cases of abuse, of stigmatization, of disempowering people and of the disenfranchising of the vulnerable carried out by the helping profession. He asserts strongly that "professionals need to become sufficiently self-aware as to acknowledge some of their own issues and needs that may intrude unhelpfully into their work with clients" (p.181). 
Watson (2000) also acknowledges the 'shadow side' of clients' and the way in which clients can emotionally, and even sexually, seduce helpers so as to keep thinking of themselves as special and retreat into helplessness and cling to a sick role in order to avoid discontinuation of care.

This 'shadow side' of the profession is also discussed by Barker (1999) who refers to the history of the profession as it emerged from the days in which 'the mentally ill' were cared for in large asylums staffed by untrained people whose role was solely to contain. Heron (1990) expressed some of these same concerns and proposed that a level of emotional competence was needed in those people who were charged with caring for some of societies most vulnerable people. He suggested there were three levels of competence and that helpers were able to move from one level to the next by doing psychological work on their past distresses so as to become more intentional rather than unconsciously reactive in their work.

The Health Force Advisory Committee in New Zealand have acknowledged there are concerns about the significant changes to the mental health workforce as a result of the shift from institutional based care to community based care as this has led to a major increase in the numbers and types of services provided in the mental health sector. This change has meant that a reorientation of the knowledge and skills is required. (Health Force Advisory Committee, 2002). As a result of these concerns, a further a discussion document was produced by an expert reference group. This document is titled Mental Health Nursing and its Future: A Discussion Framework (Ministry of Health, 2006) and its goal was to "provide strategies to move the profession of mental health nursing forward" (p.ii). It also claims to "provide a strategic direction for the future of mental health nursing that will strengthen both nursing leadership and practice within the multi disciplinary team. The document identifies and offers recommendations for addressing the following key workforce issues; nursing leadership, the role of the nurse practitioner, mental health nursing standards of practice, skills mix, clinical career pathways for mental health nurses, professional supervision, mental health nursing education, mental health nursing research nursing culture and recruitment and retention of mental health nurses. 
Interestingly, although the expert panel that made the recommendations included a representative from the New Zealand College of Mental Health Nurses, all of the other representatives represented educational institutions, District Health Boards (DHB) and other organisations that employ nurses or who are stakeholders in the sector. There was also a consumer representative who is employed by a DHB but there was no representation from the many nurses who are currently in practice and who are going to be at the end of any implementations that result from these recommendations. The document also falls short in its recommendations as it fails to address some of the key issues addressed in current nursing literature, such as what is the discourse or theory that informs the profession and how does this translate in practice.

\section{Philosophy of Care and Models of Care}

This subsection discusses the literature as it pertains to philosophies of care and models of care in relation to mental health nurses work with people experiencing suicidal ideation.

There is very little evidence of research into philosophies of care or models of care as they relate to the way in which mental health nurses work with people experiencing suicidal ideation. Cutcliffe and Stevenson (2008) carried out a literature review into this area and concluded that "there is a disconcerting lack of empirically induced theory to guide practice and even less empirical evidence to support specific interventions" and, at best, believe that nurses working with people experiencing suicidal ideation are "feeling their way in the dark" (p. 942). They also state that "The contemporary psychiatric/mental health nursing literature is clear in highlighting that all too often what suicidal people receive in the way of 'nursing care' is indeed second rate, ineffective and impersonal" (Cutcliffe \& Stevenson, p. 951).

Cutcliffe and Stevenson (2008) go further than just identifying the lack of evidence of theoretical models of care that are available to guide mental health nurses. They argue that their study provides evidence that casts doubts on the success of defensive practices and offer the opinion "that caring for suicidal people must be an interpersonal endeavourer personified by talking and listening" (p. 949).

Barker (1999) and Watson (2002) refer to the experience of mental distress in relation to people experiencing suicidal ideation. They suggest that mental health nursing needs to 
be characterized by interpersonal ways of working and yet they, along with others, have failed to put forward a comprehensive theory or model to inform the practice of those nursing people experiencing suicidal ideation.

The studies and disciplines of philosophy, psychology and psychotherapy have deep associations with mental health nursing and their influences can be seen throughout the mental health nursing literature that speaks to the theories that underpin the profession (Barker, 1999; Crotty, 1998; Watson, 2002). Barker has asked that mental health nurses examine their borrowing of ideas from other disciplines and suggests that the profession needs to develop its own discourse, yet he contradicts this by suggesting that mental health nursing needs to go back and look at the psychotherapeutically based interpersonal theory developed by Peplau (who he refers to as the mother of psychiatric nursing) in order to find direction.

The field of psychology does however have proponents who offer specific theories and models of care for working with people experiencing suicidal ideation and it might be that by borrowing and blending ideas such as these that nursing can find some direction. The works of two psychologists with alternative views on suicide will be discussed next as they are representative of the sheer diversity of approach in the field.

In his seminal work, Robert Firestone (1997) presents the view that suicide is the result of an inner or internal dialogue that has its foundation in our early childhood. He proposes that the same social milieu in which a person acquires their sense of self also causes fractures in the psyche, which can lead to a sense of de-personalization and alienation that presents as a 'voice' which can become part of a self destructive suicidal process. Firestone believes this voice is continuous, but not always conscious or spoken and open to external interpretation. His theory is that 'voice therapy' provides a process by which people can expose and come face to face with the demons they carry.

An alternative point of view was proposed by James Hillman (1997), another psychologist prominent in his field. In his work from the sixties, he challenged the view that suicide is a matter for psychiatry, or for medicine at all, by suggesting that physicians are unable to objectively take up the problem of suicide because their obligation to promote life stops them from going with their patient into an exploration of death. Instead Hillman asserted that suicide is a matter of the soul. In his work, 
Hillman explores psychological, philosophical and theological perspectives in terms of the meaning of suicide and challenges people who work with people experiencing suicidal ideation to think of suicide as one of the human possibilities and that death can be chosen. The meaning of this choice is different for each individual according to their circumstances and, when working with someone, Hillman says the premise must be that every death has meaning, so the task is to get to the "meaningful inside" (p.227).

Interestingly, in his postscript of afterthoughts written for the second edition of his book, Hillman claimed he had softened in his stance as he had come to believe that the medical model takes into public account public affairs such as contagion, epidemiology and public health which means that, in terms of therapy, the patient is seen more widely than as an individual.

This theme is also referred to further on in his postscript and more fully explains his shift from the view he held in the sixties, when he worked on the premise that suicide was a matter for an individual soul:

The individual consists of more than his or her personal individuality. Something besides 'myself 'inhabits the soul, takes part in its life and has a say in its death... We need a...definition of self as the interiorisation of community. Suicide literally 'self killing' now would mean both a killing of community and an involvement of community in the killing (Hillman, 1997, p. 198).

Hillman (1997) credits Jung with being the first theorist to refuse to classify patients into categories and to stand for the soul as the first reality.

\section{Messages about the Effects of their Work with People Experiencing Suicidal Ideation on Mental Health Nurses}

There is wide spread acknowledgement that working with people experiencing mental distress is an inherently challenging area (Roberts, 2000). In this next subsection, I discuss the messages found in the literature in relation to the effects that working with people has on mental health nurses, both in a general sense and then more specifically as it relates to their work with people experiencing suicidal ideation.

As already stated, a review of nursing literature revealed a lack of current knowledge about the impacts that working with people experiencing suicidal 
ideation has on the lives of mental health nurses. However, claims that this work is emotionally demanding and that it can have negative impacts on nurses were discovered. These claims were made almost as an aside in examples of research that were exploring associated topics such as the role of close observations in relation to working with people experiencing suicidal ideation and looking at models of care for people experiencing suicidal ideation (Barker \& Cutcliffe 1999; Duffy, 1995).

Other more generalised research has explored the emotional costs on nurses as a result of their work. These studies have often begun from the point of view of an established theoretical construct and have looked for evidence of whether or not this construct can be applied to nursing. Examples of this include literature that relates to the construct of stress and, more specifically, workplace stress in relation to nursing as a profession (Healy \& McKay, 2000; Pryjmachuk \& Richards, 2007); There are also examples of this as it pertains to mental health nurses (Ryan \& Quayle, 1999). Other constructs such as vicarious trauma and burnout have been explored separately and also in conjunction with stress (Happell, Pinikahana, \& Martin, 2003; Steed \& Downing, 1998). Ryan and Quayle suggest that psychiatric nurses are not as stressed in their work as most research indicates and that the stress that does exist is more related to organisational rather than work related issues. The findings from another study that explored the construct of emotional labour as it relates to mental health nursing in an inpatient unit suggest that the deeper the intensity of interactions, and the more variety of emotions experienced, the more emotional labour was reported (Mann \& Cowburn, 2005). This is in some way supported by Stordeur, D'Hoore and Vandenberghe (2001) who concluded that the cause of work place stress and exhaustion is multi-factorial and relates to the physical and social environment, role ambiguity, and active management-by-exception leadership.

Watson (2000) suggests that the culture of caring occupations carries an unspoken belief that staff cope no matter what and that to acknowledge anything different means that an individual might be thought of as neurotic or weak. This has also been acknowledged in police work and in other areas of employment where staff can be put under a great deal of personal pressure (Martin, McKean, \& Veltkamp 
1994; McCann \& Pearlman, 1990). Another feature common to the culture found in these areas of work is staff use of black humour as a means by which to deflect the emotional toll of working in the frontlines of death (Luke, 2004). Luke refers to this phenomenon as "gallows humour" and acknowledges that it can be helpful when it is well managed. He also identified that some individuals can fear that their use of it may indicate they have gone over the top. In relation to this, Luke placed a lot of importance on good managerial and organizational systems that support full debriefing after incidents and at the end of all shifts.

Burnout is also acknowledged as part of the culture in 'helping professions' and can result in nurses adopting a cynical attitude and feeling less empathy toward clients. This can lead them to want less client contact (Watson, 2000).

Barker (1999), Heron (1990) and Watson (2000) agree that good clinical supervision and ongoing personal development through education are necessary in order to avoid burnout and to build the relationship skills and the other key attributes of effective mental health nurses. As already mentioned, the need for good clinical supervision is also acknowledged as one of the key issues in terms of the future of mental health nursing in New Zealand (Ministry of Health, 2006). In this chapter I have reported on the processes undertaken in regard to the literature review that was carried out in relation to this study. I have also discussed examples of current literature that both inform and describe the context in which the study takes place. The next chapter moves on to explain and describe the methods and the elements of the study design processes that were followed in order to meet the aims and objectives of the study. 


\section{Chapter Three: Methodology and Research Design}

\section{Introduction}

As raised previously, the aim of this research was initially influenced by my interest in the subject that came out of my own curiosity and from conversations that I had with colleagues. This led to an exploratory literature review with the aim of uncovering what was already known in regard to the subject. The results of this search resulted in the formulation of more clearly defined aims and objectives, which led naturally toward the methodological approach chosen for the study. In this chapter I remind the reader of the overall aim and objectives of the study and explain the theoretical perspectives and assumptions that underpin the methodological approach of the study and the rationales for choosing it. I then go on to provide an overview of the study design and to report on the processes that were followed during data collection and analysis. The chapter ends with a description of the quality processes undertaken and a discussion about the rationales for these.

\section{Overall Aim}

The overall aim of this research is to describe the impact that working with people experiencing suicidal ideation has on the personal and professional lives of mental health nurses who work in acute mental health settings in New Zealand.

\section{Research Objectives}

To achieve these aims, three key research objectives were identified. These are as follows:

- To gather information from mental health nurses about their experiences of working with people experiencing suicidal ideation in relation to their philosophy of practice and that of their colleagues;

- To explore nurses' experience of working with their work place culture and organizational and professional expectations in relation to their way of working with people experiencing suicidal ideation;

- To discover what influence and impact this work has on the physical, spiritual, mental, emotional, social and whanau aspects of their lives. 


\section{Methodology}

The methodology chosen for this study was qualitative description as described by Sandelowski (2000). In this section an overview of the methodology is offered.

"Qualitative description is the methodology of choice for projects that seek to describe the phenomenon under study in it's every day terms" (Sandelowski, 2000, p. 334). "The goal of such projects is to present a full summary of the events or phenomena that has not been interpreted or influenced by any conceptual rendering of the data" (Sandelowski, 2000, p.334). The features of this method cluster around two key attributes, general qualitative inquiry and the achievement of description as an outcome of the inquiry.

The rationale for selecting this methodological approach was based on the nature of the question under consideration. This is in keeping with Denzin and Lincoln's (2008) idea that a research methodology and theoretical framework need to be constructed out of the issue under investigation. The approach was also suited as a means by which to uncover new, and establish new, knowledge in relation to the subject. Minichiello et al. (1999) add that a strength of descriptive research is that it enables health care professionals to describe what exists in practice and to classify this so that the information gained can be used by the discipline.

Denzin and Lincoln (2000), Minichiello et al. (1999), and Sandelowski (2000) place inductive research such as this (seeking to generate new descriptive knowledge rather than test existing knowledge) into the realm of qualitative research. That is, "a research paradigm that is interested in questions which involve human consciousness and subjectivity and values humans and their experiences in the research process" (Roberts \& Taylor, 2002, p. 537).

Brink and Wood (1994) and Roberts and Taylor (2002), suggest that there are three levels from which research questions can be formulated. They go on to say that questions that look at new phenomena, or that seeks to supply missing data, fall in to the first level in that they are by nature exploratory and should be formulated at a descriptive level. They identify that these questions often have two parts or 'stems', with one division naming the who, what, when, where or why part of the question, while the second stem is concerned with identifying the topic. The title of this study, 
"The impacts of working with people experiencing suicidal ideation; mental health nurses describe their experiences" followed this formula.

Roberts and Taylor (2002) suggest that issues of congruency are important to researchers who are using qualitative approaches to their work. They point out that if a methodology is a set of theoretical assumptions and a method is a process by which to generate knowledge, then it makes sense to expect that a degree of fit must exist between the two. They also state that congruency is concerned with the correspondence, agreement or fit between foundational ideas and the activity phases in qualitative research. In line with this, this study follows the principles of qualitative description as presented by Sandelowski (2000). These principles informed the project's fundamental assumptions, its design features and its methods of data collection and analysis. The ideas behind taking this approach have been supported by Roberts and Taylor (2002), Denzin and Lincoln (2000) and Minichiello et al. (1999).

The theoretical underpinnings of fundamental qualitative descriptive research are drawn from the general tenets of naturalistic enquiry (Sandelowski, 2000). As discussed previously, a strength of qualitative description is "that it seeks to avoid as much as possible the re-presenting of events by interpreting them through the filter of a particular theoretical or philosophical position" (p. 334). Instead, the researcher works to keep as close as possible to the data and to the surface of the words and events with the aim being to "allow the target phenomena to present itself as it would if it were not under study" (p. 334). Sandelowski recommends qualitative descriptive research as a method that is particularly amenable to exploring questions such as, "What are people's responses (e.g; thoughts, feelings, attitudes) toward an event?"(p. 337).

As a methodology, qualitative description differs from other qualitative methods in that its intention is to describe the phenomena rather than to interpret it. Another point of difference between fundamental qualitative description and other qualitative methodologies is that the aim is of the research is to produce a summary that describes the phenomena in the language of the everyday events and for it to be as close as possible to the meanings that participants intended (Sandelowski, 2000). One of the strengths of the methodology lies in its capacity to facilitate the researcher to use 
shadings and tones to capture the nuances descriptively in the reporting of the research findings.

\section{Research Design}

This research was designed as a small study to serve the scale and scope of a master's thesis, while generating new knowledge that is worthwhile. I understood from the outset that full data could not be achieved but, with a careful design, I believed it was possible to generate some descriptive information about the experiences of these particular mental health nurses in relation to the impacts that caring for people experiencing suicidal ideation has on their whole lives. As it stands, the scale of the study is such that the research fundamentally operates like a small pilot study.

In this next section I present the features of the design of this study in relation to their methodological rationale or bases as a table. In the first table (Table 1) I outlay the key principles and design features of the methodology used in this study under the headings of design features, specific aspects of design and the rationale and theoretical derivation. 
Table 1: Design of the study and accompanying methodological rationale

\begin{tabular}{|c|c|c|}
\hline Design Features & Specifics of study design & $\begin{array}{l}\text { Rationale and theoretical } \\
\text { derivation }\end{array}$ \\
\hline $\begin{array}{l}\text { Research Aim and } \\
\text { Objectives }\end{array}$ & $\begin{array}{l}\text { To describe the participants } \\
\text { philosophy of practice when working } \\
\text { with people experiencing suicidal } \\
\text { ideation in relation to working along } \\
\text { side of colleagues. } \\
\text { To describe how the participants' } \\
\text { ways of working fits with their } \\
\text { workplace culture, organisational and } \\
\text { professional expectations. } \\
\text { To describe the impact that working } \\
\text { with people experiencing suicidal } \\
\text { ideation has on the participants in } \\
\text { their life away from work. }\end{array}$ & $\begin{array}{l}\text { An initial literature review revealed } \\
\text { a gap in understanding about how } \\
\text { working with people experiencing } \\
\text { suicidal ideation impacts on the } \\
\text { personal and professional lives of } \\
\text { MH nurses. } \\
\text { "People cannot help but be placed } \\
\text { in, and involved in, their particular } \\
\text { time and place situations" (Roberts } \\
\text { and Taylor, 2002, p.408). } \\
\text { Study findings need to describe the } \\
\text { impact that working with people } \\
\text { experiencing suicidal ideation has } \\
\text { on mental health nurses. This may } \\
\text { lead to aspects that need further } \\
\text { investigation, exploration or that } \\
\text { may have a different application. }\end{array}$ \\
\hline Research Approach & $\begin{array}{l}\text { Fundamental Qualitative Description } \\
\text { as described by Sandelowski (2000) }\end{array}$ & \\
\hline $\begin{array}{l}\text { Data Sources and } \\
\text { Methods }\end{array}$ & $\begin{array}{l}\text { Participants: Registered Nurses } \\
\text { with more than five years experience } \\
\text { in acute mental health services (5-6) } \\
\text { Scale: Large enough to offer } \\
\text { relevance. } \\
\text { Small masters' project. } \\
\text { Data Collection: } \\
\text { Semi structured interviews of } \\
\text { around one to one and a half hours } \\
\text { using an aide mémoire } \\
\text { Record and transcribe interviews. } \\
\text { Reflective note taking. }\end{array}$ & $\begin{array}{l}\text { Small numbers in order to keep data } \\
\text { processing manageable while still } \\
\text { ensuring enough critical } \\
\text { information collected. } \\
\text { Interview processes that encourage } \\
\text { full expression of participants' } \\
\text { experiences and that allows for } \\
\text { consideration and comment on } \\
\text { cultural or contextual factors in } \\
\text { relation to phenomenon under } \\
\text { study. } \\
\text { Follows Sandelowski's suggestions } \\
\text { of how to "obtain minimally } \\
\text { theorised and otherwise transformed } \\
\text { answers to questions of special } \\
\text { relevance to practitioners and policy } \\
\text { makers" (2000.p.337). } \\
\text { Aspects of aide mémoire (interview } \\
\text { Guide)informed by Durie's Te } \\
\text { Whare Tapa Wha (1998) }\end{array}$ \\
\hline
\end{tabular}




\begin{tabular}{|c|c|c|}
\hline Design Features & Specifics of study design & $\begin{array}{l}\text { Rationale and theoretical } \\
\text { derivation }\end{array}$ \\
\hline Recruitment & $\begin{array}{l}\text { Advertised through the local branch } \\
\text { of College of Mental Health Nurses } \\
\text { Convenience sample (snowball). }\end{array}$ & $\begin{array}{l}\text { Required access to the target group. } \\
\text { Minimise risk of coercion. } \\
\text { Based on purposeful selection as } \\
\text { suggested by - Sandelowski, (2000) } \\
\text { and Minichiello et al. (1999). }\end{array}$ \\
\hline Data Analysis & $\begin{array}{l}\text { Systematic review of transcripts and } \\
\text { field notes. } \\
\text { Content and thematic coding using a } \\
\text { reading frame work and colour } \\
\text { coding. } \\
\text { Review of data in relation to the } \\
\text { research question and context of data } \\
\text { gathering in field. } \\
\text { Exploration of themes and processes } \\
\text { in relation to the aims and objectives } \\
\text { of the study. }\end{array}$ & $\begin{array}{l}\text { Reflexive interactive analysis } \\
\text { concerned with describing and } \\
\text { reporting the themes uncovered in } \\
\text { the data. } \\
\text { Unable to achieve full data } \\
\text { saturation due to small numbers but } \\
\text { sufficient to warrant consideration } \\
\text { of trends in data. } \\
\text { Focus on individual and collective } \\
\text { descriptions to understand the } \\
\text { manifest and latent data content }\end{array}$ \\
\hline Qualitative Rigour & $\begin{array}{l}\text { Participants are able to recognise the } \\
\text { description(s) and perspectives } \\
\text { portrayed in the research findings. } \\
\text { Readers are able to follow the } \\
\text { decision making trail. } \\
\text { Readers are able to transfer findings } \\
\text { into a meaningful context for them } \\
\text { outside the study setting. }\end{array}$ & $\begin{array}{l}\text { Quality measures used in project } \\
\text { Contextualisation } \\
\text { Thick description } \\
\text { Rapport } \\
\text { Auditability } \\
\text { Interpretive and descriptive validity } \\
\text { Concepts are supported by } \\
\text { Sandelowski (1986, 2000) and } \\
\text { Roberts and Taylor, (2002, p.380) } \\
\text { as appropriate for a study design } \\
\text { such as this. }\end{array}$ \\
\hline $\begin{array}{l}\text { Ethical } \\
\text { Considerations }\end{array}$ & $\begin{array}{l}\text { Ethical approval for this study was } \\
\text { granted by the Human Ethics } \\
\text { Committee of Victoria University of } \\
\text { Wellington. } \\
\text { Consideration of the overall } \\
\text { positioning and content interests of } \\
\text { the research. } \\
\text { Considerations re beneficence and } \\
\text { non-maleficience } \\
\text { Care taken to ensure that no harm } \\
\text { came to anyone } \\
\text { Care in relation to ethics of data } \\
\text { collection, gathering and reporting. }\end{array}$ & $\begin{array}{l}\text { Ethical approval for research should } \\
\text { be granted by an appropriate and } \\
\text { accredited authority }\end{array}$ \\
\hline
\end{tabular}




\begin{tabular}{|c|c|c|}
\hline Design Features & Specifics of study design & $\begin{array}{l}\text { Rationale and theoretical } \\
\text { derivation }\end{array}$ \\
\hline $\begin{array}{l}\text { Cultural } \\
\text { Considerations }\end{array}$ & $\begin{array}{l}\text { Contextual and cultural implications } \\
\text { are a 'given' and this assumption is } \\
\text { implicit in all aspects of the project } \\
\text { The study utilises Te Whare Tapa } \\
\text { Wha model as a framework to } \\
\text { structure aspects of the project } \\
\text { Treaty principles are imbedded into } \\
\text { the project }\end{array}$ & $\begin{array}{l}\text { Acknowledgement that mental } \\
\text { health nursing has its own culture } \\
\text { and that the participants practice } \\
\text { within that cultural context. } \\
\text { Treaty of Waitangi and its guiding } \\
\text { principles have become inextricably } \\
\text { linked to all that is New Zealand } \\
\text { and all it is to be a New Zealander } \\
\text { (King, 2003) }\end{array}$ \\
\hline
\end{tabular}

\section{The Research Process}

The following sections elaborate on the material presented in the previous table and report on the research processes in action.

An important aspect of qualitative description is the reporting of the research processes and decision-trail of the research. In this section of the chapter I provide a brief account of this under the headings recruitment and study context and data collection and analysis. Ethical and cultural considerations are also discussed.

\section{Recruitment and Study setting}

I begin this section by describing the setting in which the study took place. I then describe the process of recruitment before discussing the ethical considerations that were accounted for in regard to the project.

\section{Setting of the study}

This explorative descriptive study took place in New Zealand and recruited five nurses registered with the Nursing Council of New Zealand (NZNC). The nurses were required to have more than five years experience of practicing in acute inpatient or community settings and work with people experiencing suicidal ideation on a day to day basis. The participants selected worked in settings that included an adult inpatient unit, a crisis team and two day hospital areas.

\section{Sampling and Recruitment}

The sampling frame for the study conformed to the theoretical basis of convenience and snowball sampling and other general principles in relation to qualitative study informants (Minichiello et al., 1999; Morse, 1994; Roberts \& Taylor, 2002). 
Morse (1994) describes an ideal informant as being "one who has the knowledge and experience the researcher requires, has the ability to reflect, is articulate, has the time to be interviewed and is willing to participate in the study" (p.8). Sandelowski (2000) and others (Patton, 1990) also identify that the ultimate goal of purposeful sampling is to select sources that are information-rich. Minichiello et al. (1999) add that "purposeful selection must be based on the intimate relationship between the person, the event, or the situation with the phenomena under study" (p.176).

Although working with people experiencing suicidal ideation is a common feature of most areas of practice for mental health nurses, it is a phenomena that is most prevalent and at its highest intensity in the acute areas of mental health care delivery. Because of that it was decided to focus on this area of practice. This type of sampling is described by Minichiello et al. (1999) as intensity sampling.

Morse (1994) recommends a sample size of about six participants as being manageable for a qualitative study of this size and scope. Six participants were initially recruited, however, one withdrew after becoming very distracted by the tape recorder. Two attempts at interviewing were made before agreement was reached for that participant to withdraw. The decision to proceed with five participants was made after reflecting with my supervisor and acknowledging time constraints and the fact that it was highly unlikely that the loss of one participant would affect the outcomes.

As already stated, the process of recruitment was primarily driven by the need to purposefully select participants based on the likelihood of them having intimate contextual knowledge of the subject. It was also important that the process by which this happened was open and transparent.

Participants were initially recruited by responding to a flyer through the College of Mental Health Nurses advertising the research. Those nurses who expressed interest in the project were asked to let their colleagues know about the project. As discussed previously, this is consistent with the technique known as snowball sampling and is recommended by Minichiello et al. (1999) for exploratory studies such as this. It offers the researcher convenience at the risk of sample bias. In this case, the risk of sample bias was reduced by the step of having the original expressions of interest come via the flyer. The final selection of participants was made after consideration of the inclusion 
and exclusion criteria and with consideration of factors such as geographical distance and the time constraints of conflicting rosters or child care commitments.

\section{Inclusion and Exclusion Criteria}

The most important inclusion criteria was that the registered nurse participants in this study were required to have had more than five years experience of working in an acute mental health setting. The rationale for this was that they had those years of practice in which to have grown "information rich" as previously described by Patton (1990) and Sandelowski (2000). A criterion for exclusion was staff who may have been working directly with me as a colleague at the time of data collection.

\section{Ethical Principles}

This study received ethical approval from the Human Ethics Committee of Victoria University of Wellington (see Appendix one).

\section{Beneficence}

In short, beneficence is defined as 'doing good'. Put into the context of nursing research, this means that the researcher should aim to improve or benefit individuals, groups or society as a whole (Roberts \& Taylor, 2002).

Overall, this study seeks to 'do good' by describing the impact that working with people experiencing suicidal ideation has on mental health nurses. It is hoped that this description will help nurses to reflect upon their practice and then to be able to articulate the complexities of their practice in order to promote changes that will lessen negative impacts. It may also raise awareness of opportunities for personal and professional development, which could hopefully have a flow on effect into there being less defensive practice, which in turn would lead to better outcomes for people experiencing suicidal ideation.

\section{Non-maleficence}

The term non-maleficience in relation to nursing research is to do with the prevention of harm or to the minimization of harm where harm cannot be avoided. The categories of harm referred to here include harm of a physical, psychological, emotional, social, or financial nature, included in this is harm through exploitation. Several strategies were put in place in order to ensure non-maleficience. Examples of how these were built into the study can be observed in previous sections such as those on inclusion and exclusion 
criteria for participants and informed consent measures. Other strategies included the offer of petrol vouchers to cover the cost of transport for participants.

Strategies employed in the study were successful in terms of preventing harm in all of the areas where risk was identified. The negotiation of mutually convenient times for the interviews was the most difficult and this led to some time delays. This was of minimal consequence and the only person who experienced any stress over that was me. The strategy of limiting the study to those with more than five years experience proved to be valuable. The participants all spoke of experiencing positive effects as a result of taking part in the interview process and, despite some initial nervousness from one or two, they all settled easily into the process and were clearly familiar with the material that made up the content of the aide mémoire. The benefits are discussed later in chapters four and five. Allowing sufficient time for the interviews and for closure at the end was useful for both the participants and myself and was also useful in terms of data collection, as it was during these reflective moments that some of the more poignant data emerged.

So as to manage any possibility of psychological or emotional harm, nurses were required to have access to an Employee Assistance Programme (EAP) and to be undertaking regular clinical supervision. I also believed that between us there was a sufficient level of expertise and skill to enable the safe management of any feelings, thoughts and emotions that could surface during the interview process.

\section{Consent}

All of the participants were given an information sheet describing the project and were asked to give written informed consent prior to participating in the project (see appendices two and three).

\section{Confidentiality and Privacy}

Audio tapes and transcripts were coded with initials of a pseudonym. The size of the study allowed me to remember these. Participants were encouraged not to use names during the interview to ensure privacy and to prevent possible breeches of patient confidentiality. Audio tapes were checked for any identifying material prior to their being transcribed. The original audio tapes will be given back to the participants after submission of the completed thesis. 


\section{Cultural Considerations}

I begin this section by discussing cultural considerations in terms of the particular cultural context of the project and of the wider context that is Aotearoa New Zealand.

A fundamental assumption of qualitative studies is that data is bound to the particular context in which it is gathered and that people cannot help but be placed and involved with their time and place situations. They live their daily lives and yet are still connected to their past and their hope for their future (Roberts \& Taylor, 2002). This concept is implicit in the theoretical underpinnings of the methodology and is central to all aspects of this study, especially to the assumption that all of the participants have individual and unique ways of understanding and interpreting their worlds. To that end, any findings or conclusions drawn from the data can only apply as it stands for this particular group in the context of the particular setting and time in which the study took place.

I now explain how the principles of the Treaty of Waitangi and the principles of the Maori health model Te Whare Tapa Wha, as described by Durie (1998), are linked to the study design and to the methodological principles of qualitative description.

\section{The Treaty of Waitangi}

As a New Zealander, it seemed very natural for me to look to the Treaty and to other frameworks that would help to give structure to the study design and that would provide a context for the findings and discussion aspects of the project.

The Treaty of Waitangi is inextricably linked to this study because the study was carried out in the geographical, historical, social and political context of New Zealand. It is also linked by my own relationship with New Zealand, both as my place of birth and as my spiritual home. This notion was discussed by Michael King (2003) who promulgated in his history of New Zealand, that the Treaty of Waitangi and its guiding principles have become inextricably linked to all that is New Zealand and all it is to be a New Zealander. The principles of the Treaty are woven through the study and evidence of this can be found throughout all aspects of this project. This next section describes these principles in terms of how they informed this project. 


\section{Participation and Partnership}

The principles of participation (meaning being involved, Durie, 1998) and partnership (meaning working in agreement, Durie) are foundational in this study. If it were not for those early free ranging conversations held with other colleagues I might never have become interested and then invested in this project. This applied particularly to conversations I had with a senior Maori colleague who gave freely of her time. She was incredibly informative, supportive and encouraging, especially in the early stages. Other examples of partnership and participation can be found in the decision to choose a topic that would be of interest to the participants and in the implicit acknowledgement that the participants were the experts of their own worlds.

Also congruent with the principles of participation and partnership are the concepts of interpretive and descriptive validity. In this study, these principles were reflected by asking that the participants read and comment on the findings chapter. These comments are reflected on and discussed in chapter five. This process was also extremely valuable in affirming the findings in terms of establishing rigour. This is discussed further in chapter five.

The right to participate and the right to withdraw are also examples of these principles. Less obvious, but also important, was the informal approach adopted during the interview process and things such as the provision and sharing of kai (food). All of these can also be described under the principle of respect for human dignity (Minichiello et al.,1999).

\section{Protection}

Aspects of the principle of protection (meaning the same rights and privileges, Durie, 1998) are threaded throughout the ethical considerations and processes that were imbedded in the project. This principle in the Treaty also aligns closely with the principle of justice, which Roberts and Taylor (2003) describe as "the right to be treated fairly and with respect and courtesy during every stage of the research process" (p.108). The upholding of these principles was evident in processes used to select participants and in the way in which the interviews were conducted. The importance of these aspects is discussed further in chapter five under considerations to do with the methodology. 


\section{Te Whare Tapa Wha}

One of the key research objectives was to discover what influence and impact this work has on the participants' personal lives. When this was first considered in terms of the research design it became clear that any exploration into this needed to be managed carefully, both because of the sensitivity of the material that may be revealed and because of the complexity of what may emerge. My lack of experience as a researcher was also considered. In light of these issues I decided to incorporate aspects of Te Whare Tapa Wha (Durie, 1998) into the research design. The model formed the basis of the part of the aide-mémoire to do with the personal lives of the participants and it also provided the structure for presenting these findings.

Te Whare Tapa Wha is a model developed by Mason Durie (1998), an eminent Maori psychiatrist, as a means by which to demonstrate the concepts of how Maori understand health. My decision to incorporate aspects of this particular model into the study design was due to my recognition that the participants would be familiar with the concepts of this model and my belief that their, and my, understanding of these may help us as we went about exploring the subject matter.

Te Whare Tapu Wha proved helpful in the management of both of these issues. The philosophical underpinnings of this model propose that health and culture are inseparable and that there is an interrelationship between the physical, spiritual, mental, emotional, social and whanau aspects of people's lives (Durie, 1998). The key concepts adapted from are presented in the following table.

Table 2 - Te Whare Tapa Wha Model

\begin{tabular}{|c|c|c|c|c|}
\hline & Taha Wairua & Taha Hinegaro & Taha Tinana & Taha Whanau \\
\hline Focus & Spiritual & Mental & Physical & $\begin{array}{l}\text { Extended } \\
\text { family }\end{array}$ \\
\hline $\begin{array}{l}\text { Key } \\
\text { aspects }\end{array}$ & $\begin{array}{l}\text { The capacity for } \\
\text { faith and wider } \\
\text { communion }\end{array}$ & $\begin{array}{l}\text { The capacity to } \\
\text { communicate, to } \\
\text { think and to feel }\end{array}$ & $\begin{array}{l}\text { The capacity for } \\
\text { physical growth } \\
\text { and development }\end{array}$ & $\begin{array}{l}\text { The capacity to } \\
\text { belong, to care } \\
\text { and to share }\end{array}$ \\
\hline Themes & $\begin{array}{l}\text { Health is related } \\
\text { to unseen and } \\
\text { unspoken } \\
\text { energies }\end{array}$ & $\begin{array}{l}\text { Mind and body } \\
\text { are inseparable }\end{array}$ & $\begin{array}{l}\text { Good physical } \\
\text { health is } \\
\text { necessary for } \\
\text { optimum } \\
\text { development }\end{array}$ & $\begin{array}{l}\text { Individuals are } \\
\text { part of a wider } \\
\text { social system }\end{array}$ \\
\hline
\end{tabular}

(Durie, 1998) 


\section{Data Collection and Analysis}

In this next section I explain and describe the methods of data collection and analysis as they were followed in this study. The full interview process is described and then I move on to discuss the way in which the data was worked with in order to uncover the findings. I will explain how the quality of the project was ensured in the final section.

\section{The Qualitative Interview}

As implied by the title, a qualitative interview seeks to elicit information through a process that is consistent with the epistemological underpinnings of the qualitative research paradigm.

There are a number of descriptors for differing types of interviewing techniques that are recommended for a variety of reasons (Denzin \& Lincoln, 1998; Minichiello et al., 1999). Roberts and Taylor (2002) acknowledge this and argue that the label of the interview is not important; rather they suggest that the real importance lies in the way in which the researcher is able to describe the processes used.

The majority of data in this study came out of individual, in-depth, semi structured interviews, in keeping with Sandelowski's (2002) recommendations that "data collection techniques usually include minimally to moderately structured open ended individual or focus group interviews"(p.338). Roberts and Taylor (2002) describe a qualitative interview as -

... a conversation designed to encourage participants to tell their stories and relate their experiences in the deepest and richest way possible, through clear guidance on what is required, a genuine invitation to speak and communicative facilitation on the part of the researcher".(p.537)

The data for this study was collected by tape recording one in-depth semi-structured interview lasting between one to one and a half hours with each participant. This process was assisted by the use of an aide-mémoire (interview guide). Other data was collected in the form of notes taken by me of the observations that were made by the participants after the taped interview had ended and the session was winding down. I also kept a journal in which I recorded my own reflections as they occurred throughout the process of data collection, analysis, discussion and conclusions. 


\section{The Interviewing Process and the Aide- Mémoire}

The aide-mémoire used was developed to support the interview process by ensuring that I did not lose focus of the research objectives. This seemed particularly important given my lack of experience of interviewing for the purposes of research. Roberts and Taylor (2002) support the use of such a tool saying that guiding questions may be necessary in order to keep a clear direction. They caution against constructing too long a long list as "breadth of responses may be sacrificed for breadth of coverage" (p.389).

Minichiello et al. (1999) also support using an interview guide. They suggest that it is important for the researcher to be familiar with the topic and with the world of the participants and that having a conceptual understanding of the phenomenon helps with the development of questions that will be more likely to elicit the necessary relevant information.

The aide-mémoire developed for this study was constructed primarily with the aim of collecting data that would enable the projects aims and objectives to be met. As the researcher, my years of practice in the area under study meant I was familiar with the topic and had good insights into the worlds of the participants. This experience helped in terms of refining the contents of the final aide-mémoire used to guide the interview questions. As discussed previously, the decision to incorporate concepts from Te Whare Tapa Wha (Durie, 1998) into the fabric of the aide-mémoire also helped with this.

The initial review of literature pertaining to the wider context of the study was fundamental to this by providing information that helped with the initial direction and then narrowing of the focus for the inquiry.

The participants were invited to choose where the interview took place with the understanding that the venue would have to be conducive to confidential and undisturbed conversations. To this end, any interviews that were held at the participant's work place happened outside the normal working period.

In order to build confidence and to gain rapport, I purposefully took a very relaxed and informal approach and encouraged the participants to take time out when they appeared to be distracted or stuck in their responses. All of the interviews were undisturbed and, in all but one, the level of engagement was such that participants were surprised that the 
interview was over. The participant who became very nervous in regard to the tape recorder being the exception.

The aide-memoiré used in this study loosely followed Berg's (1995) model of classification for questions. The beginning questions comprised of what he describes as throw away questions. These questions were designed to help build rapport and were also used occasionally to provide space within the interview when the level of engagement with the subject matter became a bit too intense. The questions that followed were a mixture of essential questions, designed to deal with the main focus of the research and extra and probing questions. According to Berg, extra questions are those that seek to clarify or cross check information and probing questions encourage the participant to reach more deeply into the topic or to expand on significant information.

The interviews opened with a reminder of the aims and objectives of the study. The participants were then invited to read over the aide-mémoire prior to beginning the process. This concept is recommended by Roberts and Taylor (2002) as a means by which to engage with, or lead, the participant into a conversational or story telling process. After each participant had read through the aide memoir, the individual interviews proceeded with a group of questions that met Berg's (1995) classification of the 'throw away' type. This elicited individual demographical information and established the reasons why the participants had agreed to take part in the project. This information was not pertinent to the study. However, the process fulfilled the aim of placing the participants into the study and enabled the quick building of rapport.

We then moved to a question that invited the participants to look at what working with people experiencing suicidal ideation meant to them in a broad but individual sense. This led naturally to a flow of essential and probing questions that sought to identify the participant's philosophical approach, or way of working with people experiencing suicidal ideation. From there, a mix of essential, extra and probing questions were used to identify and explore the participants perspectives on the 'who', 'what', 'when', 'where' and 'why' aspects of working with this client group. The areas probed and expanded on included the participant's experience of their workplace culture, especially in regard to how they managed when they worked with nurses who used alternative 
approaches and how they worked with or managed themselves in relation to their team's approach to working with people experiencing suicidal ideation. The influence of professional and organizational expectations on the way in which the participants work with people experiencing suicidal ideation was also explored.

The next part of the interviews sought to identify the impact that working with this client group has had on the social, whanau, spiritual, physical, emotional and mental aspects of the participants' selves. Interviews were ended with questions designed not only to elicit information, but also to help us to close the process. This included attempting to identify what would help to ameliorate against the negative impacts of this work and reflecting on what counsel would they pass on to novice practitioners starting out in this area of practice. They were then invited to take time to reflect on the process and were asked to add any thing that they felt they were left with as a result of the process.

I sat with the participants in the time between their interview ending and them being ready to leave. This again helped us both in terms of closing the process down. It also ended up providing an extra and very rich source of field notes.

\section{Data Storage}

When not in use, all written material was kept in a locked filing cabinet at the researcher's home and access was restricted to the investigator, the research supervisor and the person who transcribed the audio tapes. All electronic information, such as transcripts and interview notes kept during the research phase, was kept in a password protected file and access was restricted to the investigator. Information will continue to be kept this way and safely destroyed two years after the conclusion of the research.

The original tapes made during the interviews will be returned to participants after the submission of the completed thesis.

\section{Methods of Data Analysis}

Data from this project was analysed using methods in keeping with Sandelowski's (2000) assertion that "qualitative descriptive content analysis is the least interpretive of the methods in that there is no mandate to re present the data in any other terms but their own" and is "the strategy of choice' when the aim of the research is to report findings" (p. 338). 
The primary methods of data analysis were content and thematic analysis. Content analysis was carried out using a reading tool that enabled the careful reading and rereading of the transcripts. This inductive process uncovered a clustering together of several themes that were considered and then further worked with manually by using a colour coding system in relation to the research aims and objectives.

Each transcript was read then re read several times so that I became very familiar with the style of each transcript and way in which the participants expressed themselves. I then introduced a reading tool that helped me to slowly and systematically identify any emerging themes and to discard 'the chatter'. I worked with each transcript separately and had to allow at least two days between processing each transcript so that each transcript was looked at through relatively fresh eyes. When no more themes were identified, the identified themes from each transcript were further worked with through manually colour coding and sorting them into the key themes described in the findings chapter.

\section{Concepts that Relate to Rigour and Validity}

This section discusses how the concepts and criteria that informed the quality processes inherent in this project were sustained throughout the study.

\section{Quality}

It seems as though the only firm agreement about the criteria on which to judge the calibre of a qualitative research project is that there are no set criteria (Roberts \& Taylor, 2003; Minichiello et al. 1999; Sandelowski, 1993). The reason for this has been put down to the sheer diversity of goals and methodological approaches that can be, and need to be, used in this paradigm (Minichiello et al. 2000). Sitting along side of this, warnings have been raised by what Janesick (1994) describes as 'methodolatry' (as cited in Minichiello et al. 1999) and what Wolcott (1994) describes as "posturing" and which both relate to a preoccupation with research methods at the expense of content. Sandelowski (2000) cites similar concerns and gives these as reasons for her resurrection of fundamental qualitative description as a method.

The following section discusses concepts suggested here by Minichiello et al (1999) as criterion by which to distinguish between good and bad qualitative research. The 
concepts discussed and which have relevance to this study, are; contextualisation, thick description, rapport, ethics, auditability and validity.

\section{Contextualisation}

At the foundation of qualitative research is the belief that people's behaviour is affected by their environment and experiences so, therefore, a specific behaviour or attitude cannot be investigated without taking into account all things which affect the person's perceptions and actions (Minichiello et al.1999, p.42). This concept is inherent in the project and is evidenced throughout all stages of the project from its inception to its completion. The most direct evidence for this can be found in the fundamental tenets of the methodology, in the study's aims and objectives, in the literature review and in the nature of the questions found in the aide-mémoire. It is also implicit in the findings and conclusion chapters.

\section{Thick description}

Thick description is described by Minichiello et al (1999) "as going beyond a surface description of the phenomena so that a full understanding of the setting and cultural context of what is being studied can be conveyed to the reader" ( p.42). The object of this being that a reader would understand what they would feel and experience as if they were in the same situation as the research participants. The compilation of findings from the study as presented in Craig's story successfully goes beyond a surface description of the phenomenon under study. It achieves this by placing the findings into the everyday context of the participant's worlds. It also helps to enable any reader to understand how they would feel, and what they would experience, if they worked in this field.

\section{Rapport}

Rapport refers to the establishment of a relationship with participants that is sufficiently comfortable to allow full expression of the participant's thoughts and feelings and which will assist the researcher to gain a full and accurate understanding of the phenomenon under study (Minichiello et al.1999, p. 43). Evidence that a high level of rapport was built can be found in the findings chapter. This report shows that a high level of connection was established which promoted the full and frank disclosure of information from the participants. This claim is supported by the endorsement received from the participants following their reading of the findings chapter. Factors that 
enhanced my ability to build rapport included having some degree of prior knowledge of the participants, my own in depth understanding of the phenomenon under study and my long experience of rapport building as a skill used in practice. The participants were also very practiced with their own styles of rapport building and I believe this helped them to engage.

\section{Auditability}

Auditability refers to the production of a decision trail that can be used to determine the degree to which a project has achieved consistency with its methods and processes. A high degree of auditability would allow another researcher to use a similar approach and possibly arrive at similar conclusions (Roberts \& Taylor, 2002). This chapter demonstrates auditability by clearly outlining and explaining all of the methods and processes used throughout the project. Particular to this is the table that shows the methods and processes used in the study design.

As to whether this study could be replicated and get to the same conclusions, a researcher using a similar approach would obtain similar results in so much as they too would be able to provide a summary of the phenomenon under study. However, it is unlikely they would reach the same conclusions. As discussed previously, this study seeks to describe the experiences of these particular participants, and the method chosen unashamedly places value on this as an outcome. As pointed out below, these participants work in a particular context and have their own individual responses to events. As the researcher, I too will have influenced the conclusions reached in this study. Sandelowski (2000) acknowledges this by saying that "Researchers seeking to describe an experience or an event select what they will describe and in the process of featuring certain aspects of it begin to transform that experience or event" (p. 335).

\section{Validity}

The methodological procedures used in this study, such as the care taken in sampling; the care taken in working with the transcripts, sound evidence of contextualization, the use of direct quotes from participants and participant endorsement, all contributed to proving the projects validity in terms of trustworthiness, credibility, dependability and confirmability. 
Sandelowski (2000) states that "qualitative validity in fundamental qualitative, descriptive, studies offer a comprehensive summary of an event in the every day terms of those events" (p.336) and suggests that researchers conducting such studies can be found in two forms; interpretive validity and descriptive validity. Descriptive validity describes "an accurate accounting of events that most people (including researchers and participants) observing the same event would agree is accurate" (p.336). Interpretive validity is also to do with accurate accounting but the focus moves to the meanings that participants attributed to the events (Sandelowski).

This study design ensured validity in these two areas by firstly requiring that a data analysis tool be developed and used with the aim of thoroughly and systematically eliciting the emergent themes from the data in order to represent them as valid findings. Verbatim quotes were taken from the data and thoughtfully integrated into the presentation of the findings so as to illustrate a particular point of view or a difference or similarity between responses.

Secondly, the study then sought participant endorsement by providing all participants with a copy of the findings chapter and asking them to comment according to Sandelowski's criteria for descriptive and interpretive validity. Participant endorsement is also supported by who Minichiello et al. (1999), who suggest that this verifies "that the researcher understands the participants lives" and has "told it as it is" (p. 45).

All participants found that the report met a high standard of both descriptive and interpretive validity. Their responses are discussed more fully in chapter five.

In this chapter I identified the overall aim and objectives of the study and explained the theoretical perspectives and assumptions that underpin the methodological approach used and rationales for I had for choosing it. I then provided an overview of the study design and reported on the processes followed during data collection and analysis phases of the study. I ended this chapter by describing and discussing the quality processes undertaken throughout the study. 


\section{Chapter 4: Findings}

\section{Introduction}

In this chapter I present the research findings as extracted from the results of the data collection and data analysis processes as laid out in the study design and in relation to the research objectives.

All of the findings have been presented in relation to the tenets of fundamental qualitative description, especially in relation to "presenting the facts in everyday language" and in accordance with the principles of interpretive and descriptive validity (Sandelowski 2000, p. 336).

I have presented the findings in two sections. The first section gives a straight description of the findings in relation to the objectives of the study and uses direct participant responses in the form of verbatim quotes for the purpose of illumination and accurate representation of the themes that were uncovered from within the data.

Also consistent with the tenets of fundamental description, is the presentation of a second rendition of the findings presented in the form of a composite story formulated out of the findings. Craig's story provides the reader with valuable insights into the real life experiences of the participants as they go about their work with people experiencing suicidal ideation. The story also enables the provision of description of some of the finer nuances uncovered in the data that might otherwise have been lost.

I begin the chapter by reorienting the reader to the project. This includes providing further information about me, as the researcher, and revisiting the participants and the setting in which they practice. This provides a descriptive context for the next part of the chapter.

\section{The Project}

\section{The Researcher}

Any previous (or tacit) knowledge bought with me to this project and the implications this had in terms of the selection of a methodology and the study's design have already been discussed in chapters one and three and are further discussed in chapter five. The purpose for raising this again is to ask that readers keep in mind Sandelowski's (2002) assertion that "research findings in qualitative research are variously conceived as both a process and a product in which the researcher is deeply and unavoidably implicated" 
and that "a [qualitative] finding is the sum of an irreplicable socio-cultural performance involving the researcher and subject" (p. 215).

\section{The Setting}

This project involved participants who were employed to work in acute settings within mainstream mental health services. Although it was not intended, nor considered ideal, all of the participants were employed by a single District Health Board (DHB). The implications of this are discussed in the next chapter. The settings in which the participants practiced at the time of data collection all provided services for people diagnosed as being seriously mentally unwell and were identified by the service as posing a moderate to high degree of risk to themselves or to others.

\section{Participants}

Six registered nurses with more than five years experience of working in acute mental health settings were originally sought to participate. A number of nurses expressed an interest in the study but for a variety of reasons were not able to commit to taking part. In the end, five nurses were selected. Their reasons for agreeing to participate unanimously included an interest in the subject and a desire to support the project because of that. Other reasons cited included wanting to support any nursing research, wanting to support me and wanting a chance to talk about themselves and their practice in relation to this subject. Four of the participants were very experienced (more than twenty years in working in psychiatric services) and one participant just met the fiveyear criteria.

\section{The Findings in Relation to the Objectives of the Study The Clustering of Themes}

The following section summarises thematic descriptions taken from the participants' interviews and from the field notes taken straight after the interviews. This material underwent content and thematic analysis and the following clusters were derived from this process. During the process of data analysis it was uncovered that some themes were interwoven with others. These threads will be revealed as they present themselves in the findings. For the purpose of organisation these clusters are presented in relation to the study objectives of philosophy of practice, workplace culture and personal impacts. 


\section{Philosophy of Practice}

This study sought to gather information from mental health nurses about their philosophy of practice in relation to working with people experiencing suicidal ideation. The findings from this research revealed insights into the participants' philosophies of practice, their ways of working with people experiencing suicidal ideation, how they developed this way of working and how this related to working with this client group. Insight was also gained into how the participants perceived that their philosophies of working with clients experiencing suicidal ideation fitted with those of their colleagues and how they engaged with these similarities and differences in regard to their daily practice. Similarly, the coherence of their personal philosophies of practice with organisational and other professional structures and philosophies were probed. These findings are reported on below.

\section{Philosophy of Practice in Relation to Working with People Experiencing Suicidal Ideation}

Despite using different language or different theoretical frameworks to explain themselves, all of the participants told of taking a person centred and collaborative approach in relation to working with this client group. In all cases this sat alongside a strong theme of the mental health nurses constantly weighing up the risk that they saw as inherent in this work, against the 'suicidal person's' need for autonomy in order for them to be able to grow from their experiences. The three participants who had undertaken post-graduate study positioned their philosophies in relation to key proponents in the field, for example; interpersonal (Peplau, 1988), humanistic (Maslow, 1968), Rogers, 1986) and Brief Solution Focused Therapy (Iveson, 2002). The other participants referred to themselves as having, and using, a humanistic or person centred approach to their care but did not refer to the proponents of this.

One participant talked of drawing on a number of theoretical models and gave as examples Peplau's (1988) interpersonal model, Roberts' (2000) crisis model and also talked of taking a strengths based approach in practice. The essence of this is described in the following quote:

I draw on the person's understanding and their knowing of what it is like for them...... and you would work with a person to figure it out for themselves really, but in a safe environment. Participant 2, p3 
Another participant spoke of taking a humanistic approach (Maslow,1968; Rogers,1986; Watkins, 2000) and identified concepts such as respect and acceptance as being intrinsic to practice:

Alongside risk, you also have to look at the idea that a person suffering with suicidal thoughts has choices and, in that respect, you enable them, with support and education, to make positive choices. Participant $3, \mathrm{p} 1$.

Findings revealed that the practice of the participants had developed through exposure to a variety of factors. Three of the participants described having their practice influenced through their watching of other nurses ("both good and bad") in practice and three described having been influenced by postgraduate studies. All agreed that experience on the job was the biggest factor in the evolution of their practice.

One of the more experienced nurses described the influence of post graduate studies by saying:

I do feel grounded in my practice and I feel grounded in what I know about nursing. I don't think all nurses necessarily have that. I have finished my thesis and I have done a lot of thinking about nursing...... I feel that I know what I am doing. Participant 2, p6.

Four of the five participants had extensive experience of working in this field. For these participants their years of experience appeared to equate with the degree of selfconfidence they felt in regard to their ways of working with people experiencing suicidal ideation. They had clear philosophies of practice and these were combined with their experiences to generate a sense of confidence and clarity around their interventions in practice. The following quote came from the least experienced of the participants and contrasts sharply with following quotes from more experienced participants:

I guess not having lots of experience you can doubt yourself a lot, you've got to be careful you don't get to doubt yourself too much. Participant 1, p6.

Expressions of this sense of confidence often were accompanied with revelations that the more experienced of the participants felt very little dissonance or separation between them as nurses and them as people. This was expressed well in the following quotes: 
It can be difficult to work for that amount of time in mental health and to be able to change the hats you are wearing,... you kind-of pick up almost gut instincts about people... you build the ability to build a kind of rapport, particularly in regard to people's suicidal ideas... you work alongside of them and don't have the attitude that I am the nurse and you are the patient. Participant 3, p2 \& 3.

There is a lot of me in my practice. You can't come to work and be one person and go home and be another person... think you have to bring yourself in or you are just a complete false façade. People see through it. I mean they know whether you have been a complete tosser and that you are not being real with them. Participant 4, p3

All participants used what they described as a person centred approach in terms of the action strategies they chose to use when working with people experiencing suicidal ideation. These included active listening techniques, demonstrating acceptance, a judicious use of humour, touch, being honest, being ordinary, and taking care of people's needs in terms of Maslow's (1968) hierarchy of needs. The following statements are representative of this:

Nothing... there is nothing that technical really,... it is just being able to listen, being able to listen and just let them,... that people can feel confident enough in you and trust you,... that they are not going to end up locked up". Participant 5, p2.

We look at love and belonging, and what they have that is good in their lives. For some people the love and belonging is important. Like, you know, just sitting next to someone and be close enough to touch their arm or something. I can sit and listen for a long time. Participant 3, p2.

The participants told of feeling grounded in their philosophical approach and asserted that having this helped them in times of conflict. They fall back on this when they believe that client safety is in jeopardy. One participant described as thus:

If you didn't have that strong philosophy or that strong way of thinking you'd get swept up into things and it might not be so satisfactory, so safe. Participant1, p3. 
In this subsection I have presented quotes from the participants that reveal their underlying philosophies of practice and motivations when working with clients who are experiencing suicidal ideation. It was clear from the interviews that these personal philosophies were orientating the participants' practices and offered them some stability in acting therapeutically as they engaged with this client group. I was interested to know whether these personal philosophies were supported and upheld by their colleagues or how the participants were affected by others in their peer groups. In the following subsection I report the results from this concept.

\section{Philosophy of Practice in Relation to Working with Colleagues}

The findings from this area of inquiry uncovered a number of ways in which the participants were affected by, or approached, similarities or differences in care philosophies amongst their work colleagues. Several participants commented on the comparative ease of working with colleagues when a similar philosophy of care was shared between them. Findings indicated that working in a team with a shared philosophy was something that was highly valued by all of the participants. They described feeling safer working this way and noticed there was less conflict in the team. This seemed to be more easily achieved in smaller teams. The following quote illustrates this:

I think because I work within a fairly small team... I have chosen to work with that team because the individuals that I work with do have a very similar philosophy to mine in that respect, so there is not a lot of conflict or clashing in our views as to how we treat individuals and develop their plans. Participant 3, p6.

The personal style and philosophical beliefs of medical personnel that worked with the teams was also a factor that impacted on how well the participants saw the team working:

Nurses are easier to talk to... you don't get the opportunity to sit down and debate stuff with them [doctors]. They are there and then they are gone, they are in and then they are out, they are not really team... integral team members. Participant 5, p7 
All of the participants commented on the impacts that differences in philosophies and approaches to care of people who experiencing suicidal ideation, had on their work. The strongest case in point emerged between interventionist and person-centred care. The participants unanimously linked most of theses differences in philosophical approaches to a split that they perceived between those who follow 'the medical model' and who they regarded as being more interventionist and those who take a humanistic or people centred approach and are less interventionist. Other participants noted that anxiety and inexperience factor into these differences. These perceptions were exemplified by Participant 5 who described the following:

I have seen colleagues instantly get anxious and want to intervene in a medical way, I think being suicidal ... it isn't always a medical problem, frequently it isn't. It's a crisis and crisis is not a medical term... We have to think a bit more broadly than "here is a depressed person, lets give them some pills, we need a range of approaches. Participant 5, p3.

While these philosophical positions were taken up by professionals and debated in peerrelationships often they were also created and sustained by overarching views and procedures within the organisation. In the following sub-section the participants' views about this are explored.

\section{Philosophy of Practice in Relation to Organisational and Professional Expectations}

The findings presented next reveal that most of the participants experienced a sense of unease and frustration as a result of inconsistencies and incongruent messages in regard to what organisational and professional bodies expect from them when caring for the person who is experiencing suicidal ideation. All of the participants indicated that contemporary work practices that target limiting organisational risk had impacts on their freedoms to work according to their personal philosophies of care. The following quotes from Participants 5 and 2 really demonstrate this pressure:

"We are encouraged to take a conservative approach... the whole language of risk management, it's about identifying problems, and there is no place for risk verses benefit analysis. It's very prescriptive; we don't talk about the bigger risk of contaminating people's lives by getting them into the service”. Participant 5, p5. 
Our region has a high risk adversity. The organisation says yes we are person centred and consumer driven and the environment outside of that says "no were not". Actually if you don't ensure that the risk is managed you'll be in trouble. There's a bigger system. So you've always got to be weighing up your own safety and the person's safety. There's that covering your butt thing. Participant 2, p11.

The perception of the medical model verses a person centred approach also featured in these findings with concern being expressed about how risk is managed when the organisation's documentation is formulated around the medical model and yet there is also an expectation that clinicians will take a person centred or recovery approach in practice:

It's really hard working with the medical model as opposed to a person centred model, the whole mental health system is geared toward the DSM-IV which is problem orientated as opposed to the strengths based perspective. The difficulty for me is trying to fit my way of working into a big structure that isn't orientated in that direction”. Participant 2, p4.

Distinguishing and linking personal styles in relation to organisational culture and policies also could be tricky for the participants. Most of the participants reported working with the person who was experiencing suicidal ideation in focused personcentred ways, and then having to translate their practice in a form of documentation and reporting. Conflicts about how to engage with the reporting were also evident. For instance, whether to write up the report in humanistic terms or whether to report in 'medical model' terms was a choice (influence) that the participants experienced as they engaged with the care that gave and conformed to organisational perspectives. The following quote reveals that this was also sometimes experienced as 'difference' between people and perspectives in the organisation:

Still some people are so black and white in their practice and don't have that holistic approach to care. They might think they do but is fact they don't have an overall view of it and I think they are very driven by documents and correct procedures and policies and things and can't look outside that box. Participant 3, p5. 
Findings from this section revealed that the influence of a personal philosophy of care on the strategies and intentions of the participants as they worked with people with suicidal ideation was an important feature of their practice. All of the participants reported clarity in relation to this as their personal position in relation to care, they also reported that care was easier when this personal philosophy was supported, or shared, by their peers, they reported living with difference and trying to understand or to mediate this in their practices. All of the participants found their personal philosophy of care was influenced or affected by organisational culture. They all found ways of sustaining their personal philosophy in the interactions they had with people experiencing suicidal ideation. But for some of the participants this meant that reporting of the care that was given needed significant translation to achieve and fulfil organisational expectations.

In the following section, I present the findings as they relate to the participants' experiences of their workplace culture in relation to their work with people experiencing suicidal ideation.

\section{The Work place Culture}

This study sought to uncover information about how mental health nurses experience their workplace culture in relation to working with people experiencing suicidal ideation. In this section, findings from the study revealed insights into the participants' perceptions of their workplace culture and how this related to their working with this client group. Insight was gained into how the participants engaged with the culture of their workplace and how they made sense of some of the characteristic attitudes and values that were part of this culture. Insights were also gained into the participants' perceptions of the levels of support they both wanted and received and on their perceptions on leadership. An unexpected insight into how the participants view their work was also revealed. These findings are presented next under the headings of attitudes and approach, support and leadership, and weird work.

\section{Cultural Attitudes and Approach}

The findings presented next revealed that the workplace cultures in which the participants practiced were quite different and that these differences may relate to the size of the team they worked in. Three of the participants (those who worked in large teams) told of how the cultural values of their workplace changed on a daily basis, 
dependant upon the personalities of who was working. The other two, (those who worked in the smaller teams) spoke of working in a culture with shared and more constant values.

One of the participants from a larger team told of a cultural polar dynamic that existed in their team. According to them, exactly how this dynamic played out was dependant on the personalities and attitudes of those working on any given day. The following two quotes explain exactly what they mean in the participant's own words:

There are various subcultures here and it can vary on a day to day basis. It depends who is on. Some have a hard sort cynical, world weary approach. You come on another day and people take a more psychological approach and you spend too much time talking and less time doing. Participant 5, p5.

It's some people again,... some people have a much more kind of "move them on, get them out, you know, a very quick fix. I know there are people who aren't that interested in spending a great deal of time, then there are those who want to spend too much time... there are people who struggle to take it seriously. Participant 5, p6.

The same participant admitted to having thought long and hard about the culture of their workplace and had come to the view that some people are just not suited to working with people experiencing suicidal ideation. The other participants who worked in the larger teams also made reference to personality styles that are not suited to this type of work and shared the view that more care needs to be taken when selecting people to work with those experiencing suicidal ideation. The following quote is representative of their views:

What would be the reason for dismissing someone who would be that vulnerable?... because you didn't care, and caring isn't just about doing that. At some point it is a personal quality. The wrong person in the wrong job. And that is why you have to be careful about who you employ... whereas, in the past, we haven't always been that careful. Participant 5, p 8.

Another thing alluded to by others from the larger teams, was the notion of horizontal violence as a cultural component of their workplaces. This participant sought to make sense of it by suggesting that it is a by-product of the work the team does: 
What I have noticed is that people are quite conflicted. There's a lot of what we'd call horizontal violence, there's a lot of gossip, I guess when you are working with people with high needs it get displaced somewhere. Participant 2, p7.

In stark contrast, those participants from small teams described experiencing only minor conflicts within their teams and these were explained in the following way:

Well we all have bad hair days. Participant 3, p5.

The participants from smaller teams described the culture of their workplaces in quite different terms than those from the larger teams. They did not explore the dynamics of their teams other than to say that members of their teams took a shared approach. Both participants used similar descriptors when asked to describe the culture of their team. The following quote is representative of these:

Yeah, well respect, acceptance and safely enabling,... It's just basic really. Participant 3, p7.

The two participants from smaller teams both spontaneously made references to past experiences of working in other areas where they were challenged by members of their team for not following their team's usual approach. This quote from one of these participants describes their experience:

In the past, it has been quite difficult because it depends on the people who are questioning your practice. They (colleagues) can be incredibly manipulative and try to change the situation so it reflects badly on you rather than on them. Really it should be their practice that is being looked into... they have decided that since you don't practice like they do that they should turn it all around. It's like they shift the sands..... Participant 4, p6.

In this subsection, quotes have been used to illustrate what sense the participants make of the culture in which they practice. It was clear from the interviews that the participants were influenced by the cultures of the work places in which they practiced and that this impacts on them and how they work with people experiencing suicidal ideation. In light of this I was interested to know about the support they received as they went about their work. 


\section{Support and Leadership}

Findings revealed that participants had quite different expectations and experiences in terms of the support they wanted and received from their workplace when working with people experiencing suicidal ideation. Generally, the participants who worked in smaller teams felt supported by their colleagues, however wanted more evidence of support from their immediate line manager, whereas those who worked in the larger teams were more concerned about the level of support they would receive should there be a bad outcome to their work with some one who was experiencing suicidal ideation.

The participants from smaller teams generally felt well supported by their colleagues but not necessarily by their line managers. The following quote is representative of those participants:

It is supportive within our small team but we don't see the team leader, we can make a phone call but it is not the same as face-to-face support. Participant 3, p6.

Two of the participants who worked in larger teams did not refer to the support of colleagues, rather they were more concerned with whether or not they would receive organisational support in the event of a bad outcome. One participant spoke initially of their belief that they would be supported if this happened but then appeared to reconsider this as they spoke:

I have gone through different experiences over the years and I have never felt like I will be left to my own devices. I have always felt like they wouldn't cut me adrift ... but I do know of people who have been. I was actually nervous a while ago... Another nurse I know was involved in an incident of national significance and was castigated. He was made into a scapegoat. Participant 5, p7.

These same two participants also spoke of their confidence in practising autonomously and inferred that their experience has put them beyond being able to receive support from less experienced colleagues. This next quote speaks to how they experience this.

I can always think it over and say why... you know I do tend to work with people who are less experienced so it is hard for me to look to them for support. Participant 5, p6. 
Another participant from one of the larger teams spoke of feeling unsupported by their colleagues when they were working with people experiencing suicidal ideation:

You feel like you are on your own,... when you are dealing with someone who is suicidal like that, you have to take it seriously because that is what you do, there comes a point when these people have to take it seriously and often they won't get involved, they won't, they just won't go there. So you will have to have a conversation with these people and they are cynical and flippant about it. Participant 1, p8.

This same participant believed that they would be supported by their colleagues in the event of a bad outcome. They also believed they had the support of their line manager as they had been encouraged by them to use the organisations employee assistance programme during a difficult time and had always been supported and encouraged to seek further training or education.

All of the participants wanted the difficulty of their work, and their commitment to their work with people experiencing suicidal ideation, recognised and acknowledged and more openly supported by those in upper management positions. The next quote speaks to this:

Yeah, it would be good to get some positive reinforcement, you know from people higher up in the company. They don't care about that, they don't come and tell you you're doing a good job or anything. Participant.4, p9.

In this subsection the participant's quotes revealed their underlying concerns in regard to the supports they currently received and what supports they would like to receive when working with people experiencing suicidal ideation. It transpired that participants from the larger teams can feel isolated and unsupported in their work and that their main concerns are around whether or not they would be supported by the organisation in the event of a bad outcome. Participants from the smaller teams enjoyed a high level of support from their colleagues; however they would appreciate evidence of more direct support from their immediate line managers. All of the participants would like to see more evidence of support from those in higher management positions.

In the next subsection, I present findings that relate to other aspects of the culture in which the participants work with people experiencing suicidal ideation. 


\section{Black Humour}

In this study, the findings revealed that all of the participants believed that the judicious use of black humour was an acceptable way to off-load some of the stress they saw as being inherent in this type of work. They identified that other groups of people working in extremely stressful environments such as the police and ambulance staff also employ the use of black humour to negate the awfulness of some of their work. The participants all recognised that black humour needs to be used thoughtfully and judiciously and that it can become destructive when it is used as a form of degradation.

All of the participants spoke of the need to be thoughtful of who else was around as the use of black humour may be misunderstood by those who are unfamiliar with their work environments. The next two quotes give examples of this:

I think that black humour is a coping mechanism... if we carry the stress home ... you have got to be able to switch off, particularly working with suicidal clients... I don't think it [black humour] rolls over into how the staff are with the clients. It is in a removed area and it's not really done disrespectfully. You have to think how experienced other staff are too because they might not get it for what it is. Participant 3, p 4 \& 5.

So often nurses do it because it is part of the culture, but they forget who is in the room. Whether it be a student or a trainee intern or some one like that. They kind of forget that they don't understand our culture of black humour Participant 4, p5.

The use of black humour in regard to people who were experiencing suicidal ideation in relation to a diagnosis of borderline personality disorder was raised by two of the participants. One of the participants spoke of their sense of discomfort in regard to degrading comments being expressed in a handover meeting:

A lot will depend on the client themselves, if the suicidal ideation is around borderline personality structure then the comments can cease to be funny and they become destructive and corrosive and quite difficult to be around. Participant 5, p5.

The other participant spoke of value judgements being made in regard to those people diagnosed with personality disorder. They went on to divulge that they themselves 
sometimes made jokes and made an observation in the third person that this was due to distress and of being afraid of being overwhelmed by the constancy of having to manage the conversations they have with this client group:

I think there is a certain level of dismissiveness around that ... so its like if someone has a personality disorder and suicidal ideation then it is different to someone who is acutely depressed. It's like, there is a level of who deserved suicidal ideation and the non deserved. Some times you do make jokes because you are managing these conversations all of the time. I don't think people are often conscious that there is a lot of distress. I think there is a knocked off-ness in that. Like if you were conscious to all the conversations you are privy to, you'd be over whelmed and people can't afford to be overwhelmed. Participant 2, p8.

This same participant went on to observe that this attitude toward people with a personality disorder is not always reflected in the actual interactions that nurses have with these people:

People that go, "Oh god, she's a PD"... and then I see them working with them and they are having these authentic conversations. I think it is just a way of managing working in distressing situations. Participant 2, p8.

This subsection revealed a unanimous acceptance by participants that black humour was acceptable within the context and culture of their workplaces. They all support this as a means by which to off-load and negate some of the inherent stress that is part of working with people experiencing suicidal ideation. Participants also placed quite clear qualifications around this by saying that it had to be used thoughtfully and appropriately and that care needed to be taken as it could quickly become destructive and corrosive.

In the next subsection, unexpected findings revealed as a result of the interview process are presented.

\section{Weird Work}

At some stage during the interview process, all of the participants seemed to spontaneously reflect and gain an awareness of the 'weirdness' or 'extremeness' of their workplace culture and/or of the work they do with people experiencing suicidal ideation. For some, this recognition came when they were asked to reflect on their 
workplace culture, for others this came when they were asked to look at the personal impact that this work has on them. The theme discussed here is linked to another theme that will be revealed later in this chapter.

In most cases participants used comparisons between the extremeness of their work and the work of others in wider society in order to illustrate the sense they had made of this. The following quotes are examples of how they did this:

Because what we deal with isn't the norm. People in an office don't have this happen, it's an extreme workplace and you are dealing with extreme personalities, both clients and colleagues... yeah, it is extreme. Participant 1, p6.

Like it's bizarre really, most people don't work with people who have thoughts of killing themselves. You just don't have that. People don't have these conversations. They never in their everyday lives actually talk to anyone who wants to kill themselves and yet we do it all the time, so it becomes surreal... I don't know what that means really". Participant 2, p16.

One of the participants did not experience this recognition until we had finished the interview and were leaving the building in which it took place. It was the last thing they said and it was spoken over their shoulder as they walked away. It stayed with me on my drive home:

You know, I've just got today how bloody weird this work is, ... it is just really bloody weird ... yeah ... Thanks. Participant 3, field notes.

In this section the perceptions that the participants had about their workplace culture and how they engaged with and managed this in terms of their work with people experiencing suicidal ideation were revealed.

All participants spoke of wanting more recognition of the difficulty of their work and of wanting this to be acknowledged. The participants recognised that there was a time and place for black humour as a means by which to dissipate some of the stress of the job however they all recognised that this must be used thoughtfully and judiciously as this type of humour can become destructive when used inappropriately. 
All of the participants in the study recognised the value in working as a team and wanted support and recognition for the hard work they do. The participants in smaller groups felt supported by colleagues in their teams but wanted overt support and leadership from their line management. Those in the larger teams wanted to feel more supported by the organisation when they worked directly with people experiencing suicidal ideation.

In the next section I present the findings extracted from the data in relation to the personal impacts on nurse's lives as a result of their work with people experiencing suicidal ideation.

\section{The Personal Impact}

This study sought to discover how working with people experiencing suicidal ideation has impacted on the physical, spiritual, mental and emotional and social and whanau aspects of the participants' lives. As already discussed, this section of the study was designed and informed by using aspects of Te Whare Tapa Wha (Durie, 1998). This framework was chosen because of familiarity to the participants and to most mental health nurses practicing in New Zealand.

Findings from this section of the study revealed insights into the sense that the participants have made of the act of suicide and of people experiencing suicidal ideation and how this relates to their spiritual selves. The study also uncovered insights into how the participants perceive that the doing of this work has impacted on their physical selves and on their emotional and mental selves and similarly, on the impact that this work has had on their family selves and their wider social selves. The findings are presented next under the headings of Te Whare Tapa Wha (1998).

\section{The Spiritual Domain - Taha Wairua- the Capacity for Faith and Wider Communion}

The findings revealed that all of the participants recognised that working with people experiencing suicidal ideation had impacted on them in a spiritual sense. Despite their having quite diverse ways of expressing this, all spoke of having times when they were forced to reflect on their work with people experiencing suicidal ideation from a spiritual or existential perspective. 
For one participant, working with people experiencing suicidal ideation was cause for them to reflect on suicide and their own views on the meaning of life:

Sometimes when I am out biking or something I get to thinking about the bigger meaning of all this, their lives, the purpose of their lives... It does touch because it is about your own personal values and beliefs, especially suicide... can have some quite intense internal dialogue about this. It doesn't equate to God or religion though. Participant 5, p10.

Another participant admitted to becoming distressed by what suicide may mean in terms of the persons karmic existence because they had their own strong beliefs about this. Despite this, they were also able to feel some compassion toward people who might want to end their lives:

I think we have maybe seven lives and each time you come back you have a stronger life or a different aspect of that. Therefore, when I think about people who want to kill themselves I think why? This is the only time you have of this person. I think even though your life is hell, they could have been people who lived through the 1400s or something, so it will muck up their whole carnation. You've got to do this life no matter how horrendous it is... But then I do understand it because I talk to these people...Participant 1, p11.

Only one participant made sense of their experiences of working with people experiencing suicidal ideation though their religious faith. This participant was part of a large Pacific Island family and often used the hospital chaplain as a sounding board when working with people experiencing suicidal ideation. The following quote from them explains this in their own words:

Sometimes I need to see the chaplain. I need to talk to her. I just let her know how I am feeling and what is going on for me. It helps. Participant 4, p8.

The participants all described having come to terms from a moral and spiritual perspective with people experiencing suicidal ideation and even of them committing suicide. Most had come to this by way of their experiences of being with people as they grapple with the torment that can come with a major mental illness or with facing existential issues that they cannot resolve alone or at all. The next quote captures the gist of this. 
Suicide isn't necessarily sad either, that's what I am saying. It is horrendously sad, but for the person it's not. It's relief. I remember someone who did kill themselves and I remember thinking, they've got a lot of relief, but for me there are parts of how they died and their funeral, they weren't buried how they wanted to be. But you can see why they wanted to die, it is sad that someone could feel that way but you can understand why they did. Participant1, p10.

Another participant told this and of seeing their role as a nurse as being someone who facilitates the person experiencing suicidal ideation to explore what they know about themselves and to support them to have a much bigger understanding of that. The following quote is their own particular view on this but is reflective of the essence of what most of the other participants spoke of when working with people experiencing suicidal ideation:

I do not think that there is an omnipotent god, I think there is a place for everything in the universe and there is a way of this kind of being in the universe. Suicide, there is a place for that too. It is not abhorrent. It is not like it's outside, it's not good or bad or anything. It's just like this is what is so. Then you draw on what people know themselves to actually support them support them to make a difference around that. Participant 2, p18.

The findings in this subsection gave some insights into the spiritual impacts that working with people experiencing suicidal ideation has had on the participants in this study. The findings revealed that all participants felt compelled at times to make some sense of their work from a spiritual perspective and that they had all come to their own individual conclusions. This work did not seem to limit the participants' capacity for faith and wider communion, rather it appeared to provide an opportunity to explore and reflect on some of their own spirituality as they worked along side of people experiencing suicidal ideation. The next subsection presents the findings on how working with people experiencing suicidal ideation has impacted on the physical selves of the participants. 


\section{The Physical Domain -Taha Tinana - the Capacity for Physical Growth and Development}

This subsection reveals findings into to how working with people experiencing suicidal ideation impacts on the participants' physical selves. This subsection has strong links to the next subsection which looks at the emotional impact of this work on the participants. I have attempted some separation by focusing only on findings as they relate to the physical manifestations of the impacts of this work on the participant's lives. However, this was difficult as the findings showed that the participants often linked the two together as they told of their experiences. Findings revealed that tiredness, physical tension and anxiety are inherent in this work. Tiredness was the biggest physical manifestation and this was usually linked to the intensity of their work and to anxiety. This quote reflects this well:

You know, I don't think that mental health nurses realise the impact that this work does have on us, I can go home and just lie on the couch and feel so tired, so physically and mentally drained, it is like there is nothing left inside of me. I can't even get myself up to go to the gym, even though I know it would make me feel better. Participant 3, p9.

Findings uncovered that this tension and tiredness often led to coping strategies which can impact negatively on the participant's physical health. These included overeating and drinking excessive amount of alcohol.

The following quotes:

I usually drink too much ... I know I can dwell on things, I have gone home thinking "is that the right thing to do?" ... realistically you carry risk. Participant 5, p8.

Yes, I walk it out, and if I don't do that... I think I drink it out Participant 2, p16.

Probably I eat, I talk and talk and talk, and I am a comfort eater. Participant $4, \mathrm{p} 7$.

Two of the participants also told of how ongoing stress and tension has led them to suffer from chronic intermittent gastric complaints that become worse when they are working with someone they have particular concerns about. They both told of this 
almost being helpful at times as it made them become aware that they had underlying or unconscious concerns about something. This is exemplified in the following two quotes:

I can often feel quite sick, ... feel my self getting quite tense and my chest gets tight. Participant 5, p10.

I have a dodgy tummy anyway and I feel sick sometimes and then I have to identify why I feel that way. Participant 4, p 8.

All of the participants described having times when their sleep was disturbed even if they were not conscious that they were distressed. They all told of times when they had woken in the night and wondered if the person they had seen would be alive the next day. These two quotes reflect the participants' experiences:

I will be really stressed and manic and can't sleep. Participant $1, \mathrm{p} 10$.

I can wake up and find myself thinking about what I have done and you know... was it the right thing? Participant 5, p8.

All of the participants identified the physical impact of this work on them and had their own individual ways of managing this. All but one of the participants use exercise as a means of tension and stress release. Experience has proven to them that exercise such as walking, going to the gym and going cycling releases some of the physical tension bought on as a consequence of their work. This quote from one of these participants typifies this:

Over the last six months I have walked a lot before work and that makes a huge difference. I missed a few weeks recently and I really noticed it. Exercise for me is really important, like I find it useful just for helping to think things over. Participant 2, p15.

This subsection presented findings that give insight into how working with people experiencing suicidal people has impacted on the physical being of the participants. The participants all use, or have used, unhealthy coping strategies in response to physical tensions that arise out of their work and are held in their bodies. Findings confirmed that the participants' capacity for physical growth and development has been impacted on by this work and that participants have to consciously work at reducing the 
amount of physical tension in their bodies by exercising regularly. Findings also revealed a strong link between the physical impact of this work and the impact on the participants' emotional and mental selves. The findings on these aspects are revealed in the next subsection.

\section{The Mental/Emotional Domain - Taha Hinengaro- the Capacity to Think and to Feel}

The following findings revealed that working with people experiencing suicidal ideation has impacted strongly on the mental and emotional selves of the participants. Although they all expressed themselves in individual ways, there was a universal recognition that this work has been emotionally hard on them and they all told of often being saddened by what they are exposed to. They all spoke of frequently feeling mentally tired and drained as a result of the intensity of their work and the weight of responsibility they feel when working with people experiencing suicidal ideation. Some of the participants found that the interview process provoked them into reflecting on what impact this work has had on themselves as people and how they see their worlds.

All of the participants described feeling saddened by what they are exposed to when working daily with people experiencing suicidal ideation. They also told of having to hold on to a professional objectiveness in order not to become overwhelmed by their feelings at these times. This is described well in the following quote:

There are stories you hear that make you so feel bloody sad and you think "oh my God” ... Like there has been occasions when I'm thinking "just say one more thing and I am going to start “ tearing” up... It's like walking a tight rope really, like one way you fall one way into a kind of emotional swamp and the other way is purely intellectual robot coldness so you have to kind of tread all that way... it's bloody hard. It is hard. Participant 5, p10.

Three of the more experienced participants reported that the interview process had provoked them into recognising that they had come to normalise the extreme nature of their work with people experiencing suicidal ideation. The following quote captures the essence of this recognition well:

I think you actually don't recognise the impact of it on you. You don't get that it is impacting on you because it just seems like this stuff is normal, when it is 
quite bloody abnormal really... I have just got that right now. Participant 2, pg 16.

The same participant told of their belief that this work has caused their world view to become distorted. This phenomenon was also alluded to by others during the interview process but was not expressed as clearly as it was in this next quote:

I think it skews my view of the world. Because you are in that many conversations about pain, that if you weren't careful that's what you would see around you all of the time. Participant 2, p19.

In the same vein, another participant reflected on this and who else they might have become had they not got involved in this work. Their quote reveals that a sense of loss is attached to this:

Maybe my life would have been different if I hadn't moved into this field of work... I think I have come to see the world in a far more intense and complicated way because of this... Yeah, I sometimes wonder who else I might have been. Bit sad really. Participant 3, p8.

In sharp contrast to this, the less experienced of the participants recognised the change in them selves but rather than perceiving this change as a loss, has come to accept and even rejoice in this:

Some times I think to myself, I wish I didn't do this \{work\} because I wish I didn't know about this stuff. But I have, and I enjoy it and I want to keep doing it. It's part of me now. I feel like... "Bring it on!” Participant 1, p14.

Four of the participants spoke of the benefits of regular clinical supervision in helping them to manage the mental and emotional impact that this work has on them and they all acknowledged the benefits of debriefing and of talking about their work with colleagues in order to help dissipate some of the distress they experienced as a result of their work. The participants who spoke of supervision told of using this to gain an external perspective on their work that helped them to make sense and put away some of the more troubling aspects of their work. This was particularly useful when they had noticed niggling thoughts about have missed something. This next quote is how one participant expressed this: 
I think supervision is a way of externalising some of it. I find it useful to be able to think out loud and to bounce ideas around. It lets you be confident in the decisions you have made or to think about how you might do it differently next time... Participant 5, p8.

The participants were unanimous in thanking me at the end of their interviews and fed back that the process had helped them to reflect on their practise recognise what impact this work does have on them. This is quote is representative of this:

Thanks... You know I didn't know I thought about it all like that. We should be made to do this more often really, it should become part of our day. Participant 3, field notes.

The findings uncovered in the previous subsection provided insights into how this work has impacted on the participants emotional and mental selves. They reveal that working with people experiencing suicidal ideation has affected the participant's capacity to think and feel by influencing the way in which they view their worlds and who they are as people. This has both positive and negative impacts. The following subsection presents findings that reveal what impact working with people experiencing suicidal ideation has had on the participant's family and social selves.

\section{The Whanau/Social domain- Taha Whanau - the Capacity to Belong to Share and to Care}

The findings from this subsection revealed that working with people experiencing suicidal ideation has impacted significantly on the participant's family and social selves. The participants all reported feeling socially alienated as a result of the work they do. Most reported experiencing this same sense of alienation at home. Those participants with children told of feeling guilty that they had nothing left to give to their families at the end of the day. This applied to partners as well.

The participants who told of experiencing feelings of guilt in relation to not having much left to give to their families at the end of their day, described feeling conflicted by their need for time to themselves and their desire to be there for their family.

The following quotes are examples:

I'd have had one to one with someone and I go straight home and get on with dealing with the kids and find I have burst into tears, or I go off on my own 
and don't talk to anyone for while... so of course it impacts on them. Participant 1, p11.

I think it does impact on the family... I have been busy all day with someone who needs high input and you go home and they all say "what's for dinner?" ... Sometimes the family know that... Sometimes you just need time out and it's hard on your family. Participant 4.p 8.

Two of the same participants reported that they have had time of being fearful or over protective toward their children in terms of the child's potential for committing suicide:

A few times in the past when my son was younger and I'd have seen a kid... I'd get a bit fearful that might be a possibility for him... it can impact on how you might manage things where as other people might not even think about it. Participant 2, p18.

Their children can also experience some of the stigma that is associated with being a mental health nurse:

They do get grief from their friends though, about having a mother who is a lunatic attendant or what ever... I worry about them a lot more, yeah. A lot more. Probably far too much. Read too much into things maybe. Participant 4.p 10

Some positive impacts were also described:

I think at some point it's been helpful for the children, they can talk to their peers about youth issues and all that. They would know what to do with some one who was suicidal. Participant 4, p10.

The strong sense of social alienation reported by this group of participants was one of the strongest and most emotive of the themes to emerge from this project. This sense of isolation was universally identified by the participants as being evident in the interactions that they have with people in wider society. All of the participants describe only really feeling comfortable when they are out socialising with other mental health colleagues. The following quotes represent the participants telling of this: 
It becomes very difficult to socialise in different environments, people will move away from you when they know what you do. It's really obvious too. They don't do it discreetly. Participant 4. p10

There are times when you experience something so outrageous that if you told them about it you know they wouldn't get it ...not get it at all. You can't just go in and say "guess what happened today!"... whereas you can with people who do the same work. Participant 5 p. 9

I think in terms of socialising with other people you end up having quite superficial relationships because they are just not going to understand you really, that's why we all end up socialising together. Participant 3, p9.

Alongside of social alienation, four of the participants told of times when they experienced the same sense of alienation when spending time with their family. The participants described this differently but all reported that this sense of alienation was distressing for them. They told of feeling almost protective of their families. The following quote was typical of this:

You work in an environment that seem so alien from theirs... you wouldn't want them to know really. Participant 2, p18.

For those with partners, this sense of alienation was something they sometimes experienced in their relationships. One participant explained it like this:

I don't share anything because there is such a big gap between what we do.

The impact is that I don't get to share my difficulties or my victories with him.

He doesn't know this part of me. Participant 2, p17.

A poignant example of this sense of alienation was expressed by the following participant:

You can just be watching TV with them and feel like you don't belong, that you could almost contaminate them with what you know and what you have seen. Participant 5, field notes.

These next findings reveal some of the participants' spontaneous reflections on working with people experiencing suicidal ideation. These findings came mainly from data that was collected at the end or toward the end of the interview process when the 
participants were relaxed and contemplative. They seemed best placed under this heading.

Two participants spoke of the sense of privilege they feel as a result of working with people experiencing suicidal ideation. The following quotes exemplify the poignancy of their feelings and the sense of privilege they experience in regard to their work with people experiencing suicidal ideation:

So time stops basically. Nothing else can be going on because it is about what they are experiencing at that moment, time stops. I feel that as a nurse it is a huge privilege because you are involved with them at a time when nothing else matters. It's like childbirth really. Participant 1, p2.

I don't think it is a terrible thing that people get to feel this way... It is a privilege to work with people who are able to share this with you, whereas in your normal life, you wouldn't get that....Also you can feel proud of yourself that you are able to be in these conversations because they are big conversations... That you can actually be with people who think like this. Participant 2, p20.

Most also reflected on the lighter moments of working with people experiencing suicidal ideation and of the sense of comradeship that working in extreme environment fosters. This was particularly evident in this composite quote taken from the field notes: You know it can be just so weird or so sad that it just occurs as being really funny... and you can end up laughing together when it's over. It might even be about something that is not to do with the person you've seen. It might be to do with something the psychiatrist said that was just so off the mark that the person looked to you both for reassurance or something. You don't even look at each other at the time but then you get into the car when it's all over and off you go laughing hysterically. I have laughed till I cried a few times. ... Yeah, it's good when that happens. It's those funny moments and the moments when you know you've actually connected with someone, it [the work] hooks you in really. Participant 5, field notes.

This subsection presented findings that gave insights into the impacts that doing this work has had on the participant's family and social selves. The findings revealed that 
the participant's capacity for belonging and sharing is severely impeded by a sense of alienation that they believe comes about as a result of the way in which they and others perceive their work. The findings also revealed that the participants all believe that working with people experiencing suicidal ideation has vicariously impacted on their families as well. Along side of that however, the findings revealed that some participants also experience a sense of privilege and feel some pride as a result of their work.

\section{Craig's Story}

This presentation of a second rendition of the findings in the form of a composite story provides the reader with valuable insights into the real life experiences of the participants and their practice worlds as they go about their work with people experiencing suicidal ideation and reveals some of the finer nuances uncovered in the data that might otherwise have been lost.

Craig is a community mental health nurse who works as a crisis clinician in a community mental health team. He has been practicing for twenty-two years and has prior experience in a variety of mental health settings including forensic services, acute inpatient ward and as a case manager in a community mental health team. He has also worked as a nursing lecturer in a tertiary education setting. He has a master's degree in nursing and is still engaged in further education. He is married and has two teenage children.

The team Craig works in provides the mental health crisis response service for a medium size city with a number of satellite suburbs. Most referrals to the team come through the police, the emergency department at the local hospital, via other community mental health teams or through direct contact from the public. The team is funded to provide a crisis response to those people who are triaged as being significantly at risk and who need to be seen by a clinician within between two to twenty four hours, depending on their level of risk.

Craig works eight-hour shifts that rotate over 24 hours and are four days on and two days off. Today he is on an afternoon shift. Craig has spent the morning studying and has cycled into work. There are three others on this shift, one person is coordinating, which involves triaging all calls that come through to the team and then organising the 
level of response that is required to meet the needs of the person as they relate to the level of triage. Craig has sat through handover and is aware that a man is waiting to be seen in the police cells. He is assuming that he will be going to assess the man, who was picked up by police after his wife had found him in their garage about to hang him self. The background information was that this had been in the context of relationship difficulties and that the man had been drinking. During the handover the triage nurse had described the man as "another attention seeking loser in need of a spinal transplant". Craig has experienced conflict with this nurse before and wonders if this is an attempt to provoke him into yet another episode of open dispute. He is also aware that the triage nurse will, most likely, employ delaying techniques before sending him to the job as this particular nurse seems to like to make the police wait for a response. He goes into the triage office and picks up the documentation related to this job and tells the triage nurse he is going off to do the assessment. The triage nurse grunts "what ever". Craig invites the student who is currently placed with the team to come with him to the assessment. He goes in to tell this to the triage nurse who says, "more fool you". The student was present in the handover and also hears this.

They go off to the police station. On the way the student asks him about why the triage nurse said what he did. Craig decides to tell her about the phenomenon of burnout. She asks why the nurse is still working in the area and Craig tells her that this nurse used to work in one of the old institutions and has never kept up, or been made to keep up, with current understandings of mental health nursing. He also points out that nurses are like every other group in that there are people who end up being the wrong person in the wrong job. Craig says that the work they do is inherently stressful and that black humour is sometimes a useful tool for dissipating some of the distress caused by constant exposure to people experiencing suicidal ideation. He adds that not every one is emotionally suited to working with people in distress and that sometimes people get trapped in jobs because they have families to support. He encourages the student to take time before choosing an area of nursing in which to specialise.

They arrive at the police station and are shown into a holding cell. The man in the cell smells strongly of alcohol and has visibly raised ligature marks around his neck. Craig immediately asks the attending police officer if this man has had a medical assessment. The reply was negative so Craig asks the policeman to request an ambulance to take the 
man to the emergency department. The man in the cells tells Craig that he is not going to the emergency department and that Craig can "go and F..k himself" if he thinks he will make him. The policeman says to Craig that he will stand down the ambulance. Craig explains to the policeman that the man could be in danger of having his airway occlude as a result of swelling of the larynx and asks if the police will assist him to get the man to hospital. The Senior Sergeant on duty tells Craig that they are too busy and that the man will have to wait till they have a police officer available. Craig again explains the gravity of the situation to the senior sergeant. The Senior Sergeant concedes and a policeman assists ambulance staff with transporting the man to hospital. He requires restraint with handcuffs to prevent him from assaulting the ambulance staff. Craig informs the man that he is in the care of the police under prevention of suicide legislation.

On arrival at the hospital Craig informs the man that he is now being held under section 111 of the Mental Health (Compulsory Assessment \& Treatment) Act 1992 (MHA) and that he is arranging for an assessment under the powers of that Act. He calls the man's wife and tells her of her husband's whereabouts and gets further details of events prior to the man's suicide attempt. It unfolds that this man and his wife have been trying to start a family through IVF treatment and that their last and final attempt had proven to be unsuccessful. The man's wife told of a three-year period of them trying to conceive, during which her husband had seemed to be the stronger of the two. His attempt to hang himself had come out of the blue as far as she was concerned. He had never had any previous periods of depression and had a successful fish mongering business. She asks if she should call his family and whether she should come to the hospital. He and she both come from large Italian families. Craig decides to talk to the man prior to giving the go-ahead for this and, after checking she was coping, he and the student made their way into the cubicle.

The ear nose and throat specialist has cleared the man for now but wants him admitted for observation overnight. Craig is asked to assess the man's risk of suicide in relation to this. The man is at first reluctant to engage with Craig and, after Craig reveals that he has talked with his wife, the man becomes tearful and curls up into the foetal position, facing away from Craig. Craig gently asks if he would like his family to come to support him. The man doesn't verbally agree but his sobs become audible so Craig 
decides to go ahead and contact the man's family. On his way to the phone Craig explains to the student that family supports will be very important to ensure the safety of the man and that having family present will help Craig in his assessment of the man's level of risk. He calls the man's wife and she agrees to pick up his parents on her way in to the emergency department.

When the family arrive, the man breaks down again and Craig and the student watch as the man's elderly father gets onto the bed and holds him close as they weep together. At the sight of this, Craig becomes a bit tearful himself. He signals the student and they leave the family in private. He returns later to check in on the man. He informs him that he has stopped the Mental Health Act process and the man lets him know that his brother has come in and will stay overnight. Craig talks to the brother and discusses his concerns about the man's safety. The brother agrees to stay with the man at all times and will contact staff if he needs to have a break. Craig then arranges for a time to visit with the man tomorrow prior to his discharge. He and the student head back to base. The student is very quiet and Craig feels a bit embarrassed that she had seen him become tearful. He decides to stop and to shout her coffee so they can debrief.

Over coffee, Craig explains the rationale for his interventions and is surprised when she asks him how he copes with working with people experiencing suicidal ideation. He told her that he sometimes he does not cope but that over the years showing his emotional response to a situation such as this had become okay for him and that he now regards it as normal to become upset when faced with sad situations. He explained that, for most people, suicidal ideation is an expression of the level of distress they are experiencing and that it usually meant that they were having difficulties with expressing or dealing with their distress in other ways. He told her that he usually finds it helpful to let people see that he has become upset because it validates their own feelings and helps with building a connection with them. He went on to explain that he believes the most effective thing he does with people experiencing suicidal ideation is to take time to really listen to them and not to presume he already knows the answers from what he hears in handover. He explains the risks he believes are inherent in assuming things unless you checked them out for yourself. He said that in the case of this man he needed to be able to really engage with him in order to be able to fully assess the ongoing level of risk and that this would not be possible while the man was still 
intoxicated. Craig explained also that the man and his family needed some time to come to terms with what had happened and that he believed that carrying out an assessment at this time was insensitive. The student was able to understand how it was appropriate for both the man and his family to have family care for him, especially given their culture.

When they get back to the base and Craig and the student go about filling out the documentation required by the organisation. It takes time, as Craig has to explain how he translates what he has done in practice into the language of the medical model used in the forms. He tells the student that in the past he would have gone to a similar situation and carried out a formal, psychiatric evaluation, which quite often would have led to an admission. He explained that a full psychiatric evaluation asks the clinician to look for evidence of a mental disorder and that this often meant that responses by people to normal life events were interpreted as symptoms of mental illness rather than seen as expressions of distress. He also said that people often experienced this process as dehumanizing and that they could become quite aroused as a result. He tells the student that university studies and working with other nurses who share similar philosophies of practice has led him to take a person centred approach and to follow crisis theory in terms of how he assesses and evaluates people and the situations they find themselves in. He believes this way of working compliments his background as a psychiatric nurse. He takes time to explain the Roberts' Model of Crisis Intervention (2000) as it applied to the person they had just seen and points out the evidence he had for believing that the man was going through a 'normal' life crisis.

He then takes the completed documentation through to the coordinator for the next handover and the coordinator asks him how the back surgery went. Craig feels himself get angry so he leaves but returns later to remind the coordinator that it is prudent to ask about ligature marks when triaging information regarding a hanging attempt. The coordinator tells Craig he has been doing the job for long enough now and that he doesn't need to be told how to do it. He finishes with a "thanks anyway".

Craig catches up with another colleague and they decide to go and have a drink together before they go home. When they get to the pub the barmaid asks them how their night has gone so far. Craig tells her that they have just finished work and she comments that 
they must be cops to be finishing work at that time. Craig's colleague mutters "something like that" and quickly turns away to defect any further questions. They go and sit down and have a bit of a laugh about how they would have explained their night to the barmaid. They agree their work is just too extreme for most other people to understand. Craig then tells of his experiences with the coordinator. They both speak for some time about their sense of frustration at the lack of consistency in the team's approach to people who are experiencing suicidal ideation and at the attitude that some people in their team display toward those people. Craig's colleague believes that the person who was coordinating the team that night would not behave as he does if there was good nursing leadership in their team. Craig agrees. They sit quietly together for a time then call it quits.

As Craig drives home he finds himself thinking about the man and why he would suddenly consider suicide. This time of contemplation is not unusual for Craig. He has thought long and hard about suicide and has come to understand this conceptually in relation to his work as a nurse and himself as a person. Craig still struggles with this at times as he believes that some people need to look at and consider death in order to choose to fully live their lives. He believes that his role in this is to support the person their exploration by becoming connected to them and standing alongside of them, while they explore what this means. As a result of his work, Craig has been forced to consider his own beliefs about suicide. He has come to recognise that a sense of separation from his own anxieties about death and his anxiety about the consequences of a patient's death on him professionally is absolutely necessary if he is to work effectively with this client group. He becomes aware again that none of the other people in his life will ever understand what he experiences as a result of his need to manage all of this. He feels very alone for a while and then gets to thinking about the man. Craig is confident that this man will not end his life as he had observed the strong emotional connections that this man has with his family and noted his capacity to accept their love and support. His gut feeling is that the man has held back from dealing with his own feelings in order to support his wife and that these feelings have erupted out of him with the help of quite a bit of alcohol and left him feeling very vulnerable.

Craig arrives home and finds himself looking in on his kids on his way to bed. His son has worn his street clothes to bed again and Craig has an urge to wake him up and tell 
him to get into his "bloody pyjamas". Then he remembers the man he has just seen and, instead, kisses his fingertips and touches them to his son's forehead and smiles when he thinks of the horror his son would feel if he knew about this gesture.

He joins his wife in bed and suddenly becomes aware that he is absolutely exhausted. He wants to cuddle up to his wife to feel her warmth but is still in the separate place he goes to sometimes and, when he is there, he tries to protect her from what he knows. He's also glad his wife is asleep as this means he can avoid her well meaning "How was your day?" question. He rolls over and starts thinking ahead to tomorrow. Then he catches himself - the only certainty in his job is that he never knows what the next day is going to bring and, for now, it that the challenge of that uncertainty that appeals most to him.

\section{Summary}

In this chapter I began by providing information about the participants, the study location and information about me, as the researcher. I moved on to present the findings as extracted through the processes of data collection and analysis and in relation to the objectives of the study.

I then presented a composite story formulated with the aim of rendering the findings so as to reveal the finer nuances of the practice worlds and the everyday experiences of the participants in relation to their work with people experiencing suicidal ideation that might otherwise have been missed.

Sandelowski (2000) asserts that "Qualitative descriptions have as their goal a comprehensive summary of events in the everyday terms of those events" (p.334). I believe that the findings as presented here meet this goal. The participants' responses to reading this chapter endorse this claim.

In the following chapter, I discuss the findings in relation to the aim of the study and offer reflections on the implications of these in relation to practice, policy and future studies. 


\section{Chapter 5: Discussion, Implications \& Conclusion}

\section{Introduction}

This study commenced with the overall aim of exploring the impacts that working with people experiencing suicidal ideation has had on the personal and professional lives of mental health nurses who work in acute mental health settings in New Zealand.

Three key objectives were identified in order to provide the structure and focus for the inquiry, these were:

- To gather information from mental health nurses about their experiences of working with people experiencing suicidal ideation in relation to their philosophy of practice and that of their colleagues;

- To explore nurses' experience of working with their work place culture and organisational and professional expectations in relation to their way of working with people experiencing suicidal ideation;

- To discover what influence and impact this work has on the physical, spiritual, mental, emotional, social and whanau aspects of their lives.

In this final chapter, I discuss the findings in relation to the aim and objectives of the research. I then reflect on the design and method of the study in terms of rigour and its limitations before discussing the implications of the study as they relate to practice, policy and possible research endeavours. I close by offering some recommendations.

\section{Relevance}

The findings taken from this study are especially relevant within the context of mental health nursing in New Zealand because the country's rate of suicide is among one of the highest in the western world." (Associate Minister of Health, 2002, p.3). Further, nearly five hundred people die every year and more than five times that number are hospitalized as a result of a suicide attempt.

The management of a suicidal crisis as a result of a suicide attempt is the most frequently encountered mental health emergency and is acknowledged as being amongst the most difficult and frightening challenges faced by mental health professionals (Roberts, 2000). In this country, the most likely person to carry the first assessment of a person following a suicide attempt is a nurse and they will often continue to engage 
with and coordinate much of the care for these people who can still be highly at risk of completing suicide (Mental Health Commission, 2001).

The knowledge gained here adds to the body of existing knowledge concerned with nursing people who are experiencing suicidal ideation and the findings from this study also hold relevance for other professionals and groups engaged in working with people experiencing suicidal ideation.

This study generated new knowledge about the impacts that working with people experiencing suicidal ideation has on mental health nurses. It does this by offering meaningful insights into the personal and professional lives of the participants and has relevance because this implies that other nurses working in similar practice areas in New Zealand may also be experiencing these same impacts.

The outcome of this study have direct relevance for other nurses who work in the same areas of practice as, not only does it provide a basis from which they can consider themselves and their own situation in relation to the findings of this study, it also offers recommendations that, if followed, may help to mitigate against some of the negative impacts identified here.

\section{Discussion in Relation to the Findings}

I commenced this study with the aim of learning about the impacts of working with people experiencing suicidal ideation in relation to the personal and professional lives of mental health nurses. I became interested in this because of my own experiences and observations and because of conversations held with others nurses, both of which had led me to believe that working with people experiencing suicidal ideation caused high levels of expressed and unexpressed anxiety and distress in individual nurses and in teams.

I was also aware that my work with people experiencing suicidal ideation had affected aspects of my personal life and, again, through conversations with others, believed this was not an uncommon experience.

A literature review revealed that little or no research has been carried out into the impacts that this type of work has on nurses in practice. Other studies had explored associated phenomena, but none had focused specifically on the impacts that working 
with people experiencing suicidal ideation has had on the whole lives of mental health nurses practicing in New Zealand.

The findings from this study revealed that the participants believed that almost all aspects of their lives have been significantly influenced as a result of their work with people experiencing suicidal ideation. Although there were individual descriptive variances, there was universal agreement among the participants that this work is emotionally and physically hard. It is also tiring and stressful and this, along with other extraneous factors, led these particular nurses to sometimes experiencing an internally felt sense of isolation within their workplace and a sense of alienation from wider society and in some cases, even from their families. The factors that influenced this included having to adapt their approach in relation to the culture of their workplace in relation to organisational approaches and to the differences of approach to this work from some of their colleagues.

All participants identified the benefits of having a clear philosophical position to refer to in terms of their work with people experiencing suicidal ideation and believed that debriefing and clinical supervision had a role to play in mitigating the negative impacts of this work. The participants also told of feeling supported by some of their colleagues and described experiencing a sense of comradeship when they worked alongside of those who shared their philosophy of care. It was also revealed that the participants preferred to socialise with their colleagues as it was in these situations that they experienced feelings of being fully understood and accepted.

The participants also described having come to some sort of existential or spiritual understanding in regard to their work with people experiencing suicidal ideation. For most this understanding was positive and had transferred into aspects of their own lives and this may never have occurred had they not been exposed to this work.

\section{The Professional Impacts}

In this next section I discuss the findings in relation to the key research objectives.

\section{Philosophy of Care}

The first key research objective was to gather information from mental health nurses about their experiences of working with people experiencing suicidal ideation in relation to their personal philosophy of practice and discover how this related to 
working alongside of colleagues and to their management of organisational and professional expectations.

The findings from the study revealed that the influence of a personal philosophy of care in their work with people with suicidal ideation was an important feature of each participant's practice. The practice of all the nurses in this study was informed by a personal philosophy of care that was based upon humanistic, interpersonal and personcentred principles and, for the participants; this philosophy of care provided the foundation from which they approached their work with people experiencing suicidal ideation. Importantly, this philosophy of care formed the basis for their decision making in terms of risk management. It also influenced their interactions with colleagues and helped them to mediate when faced with having to manage conflicts over differences in approach.

Most participants spoke of how an adherence to following the medical model led their colleagues to take an interventionist approach when they worked with people experiencing suicidal ideation, which the participants believed had the potential to reduce the capacity of the person to develop resilience. Most of the participants found this way of working hard to accept. These same participants were also concerned about how inconsistencies of approach in their teams impacted on the way in which their team supported one another. They believed that the resulting discord influenced the way in which the team interacted with people experiencing suicidal ideation.

This adherence to the medical model also impacted on the participants in terms of having to practice within the context of inconsistent messages in regard to their organisation's approach to working with people experiencing suicidal ideation. On one hand they are encouraged to take a person centred approach and to follow recovery principles and on the other hand they are required to translate what they have done in practice into the language of the medical model in order to meet documentation standards.

There was a sense that a shared philosophy of care seemed to be more easily achieved in smaller teams and other factors, such as the presence of good nursing leadership might reduce chances for conflict. 
The nurses' philosophies of care had developed over time and the participants believed they were influenced by observing the practice of others, by their experiences of working with people experiencing suicidal ideation and, for some, by undertaking studies at a post graduate level. The findings also revealed that differences in their levels of experience might have impacted on their sense of confidence and that this, in turn, could determine their level of autonomy in practice.

\section{The work Place Culture}

The second key objective of the research was to explore the nurses' experiences of working with people experiencing suicidal ideation within the culture of their workplace.

The participants described experiencing their workplace culture as something that both supported and challenged them.

Conflict around strict adherence to the medical model of assessment and treatment was again described by all participants who felt as though working from this paradigm restricted how people experiencing suicidal ideation were treated and that practising solely within this paradigm was unsafe in terms of practice.

Participants from the smaller teams described feeling supported by their teams and spoke of observing very little conflict when it came to working as a team with people experiencing suicidal ideation. They ascribed this to the team taking a shared philosophical approach. They believed this was easier to achieve in smaller teams.

The participants from the larger teams described that their work was easier when they worked with others who shared their philosophy of care. They also described times of experiencing horizontal violence and of being very challenged by the attitudes and behaviour of some of their colleagues who did not share their philosophy of care. They described this as a sort of cultural polar dynamic that played out in ways that was dependant on the personalities and attitudes of those working on any given day.

Black humour was a characteristic of all of the participants' workplaces and they believed it had a valid purpose in terms of being a vehicle for the expression and dispelling of the distress and frustration that result from working with the uncertainty inherent in this type of work. The participants also believed that the use of black humour had to be well considered and that it was something that could easily become 
damaging if used inappropriately, such as in front of students and when it became persecutory and derogatory and was related to people who had been given the diagnosis of borderline personality disorder. One participant reflected that they themselves had sometimes made jokes that were not okay and connected this behaviour to their feelings of distress and of feeling overwhelmed by the constancy of having to manage the levels of intensity they experience in their conversations with this client group.

All of the participants described that, during the interview process, they had become aware of the nature of their work from an outside point of view and that this had made them see their work with people experiencing suicidal ideation as extreme, and weird, and as something not easily understood by outsiders.

\section{The Personal Impacts}

The final key objective of this study was to discover what influences and impacts on the physical, spiritual, mental and emotional and social and whanau aspects of the lives of mental health nurses as they work with people experiencing suicidal ideation. The findings revealed that this work has impacted on all aspects of the personal lives of the participants.

The findings from the study uncovered that the participants of this study have all had to confront and make sense of their own individual and personal concepts of and about suicide and suicidal ideation. They all spoke of having felt feeling compelled at times to reflect upon their work from a spiritual or existential perspective and of learning to come to terms with people's experiences of suicidal ideation in relation to this understanding. Only one spoke from a religious perspective, the others all described this phenomenon in wider more existential terms. Most told of having come to this understanding by working alongside people and through listening as the person grappled with the torment they were experiencing as a result of a life crisis, a major mental illness, or with existential issues that they could not resolve for themselves.

The participants all seemed to have developed an innate compassion for people experiencing suicidal ideation and had the ability to sit with, or just be with, the person as they struggled to make sense of their experiences. In this sense, the participants work with people experiencing suicidal ideation seems to have helped them in their 
development of a fuller capacity for faith and wider communion in terms of their work, and for some in terms of their lives in general.

The findings also revealed that being physically, emotionally and mentally drained and exhausted and experiencing constant tension and anxiety are inherent in this work. The participants described the work as hard, and told of usually having no energy left at the end of their working day. All have found that some form of exercise helped to reduce the physical and emotional tension they experience. Most told of times when they over ate or used alcohol excessively as a means of coping. They spoke of this as being unhealthy and unhelpful in the longer term. Their descriptions showed that the participants have to consciously manage the impacts that come with working with people experiencing suicidal ideation and that this is not easy.

Each of the participants became aware during the interview process that their experience of this work had changed them as people. Some wondered about who they might have become had they chosen another profession or another area of practice. For one this realisation was experienced as a sense of loss while for another it was accepted as part of who they are now and is something that is okay and should be celebrated.

Experiencing feelings of being alienated from society and, in most cases, having times of feeling alienated from their families as a result of their work was one of the most distressing impacts described by the participants. Alongside of this was the recognition that their work has impacted vicariously on their families and partners. Descriptions about the effects of their work on them and their families were both negative and positive, but, for the most part, it highlighted that doing this work meant that the participant's capacity to belong and to care and to share was often compromised.

The realisation of the extreme nature of their work was also some thing that was uncovered during the interview process and I believe this is interrelated to the participants' experiences of social isolation and their feelings of alienation from the wider world and from their families.

The chance to talk about their work with colleagues and having good clinical supervision was valued by all of the participants as they found it helpful in terms of stress reduction and in terms of making sense of their work. 
An unexpected finding was that the participants all spontaneously observed that the process of participating in the study had turned out to be a reflective process for them and that this had had revealed to them hitherto unknown aspects of themselves and their work. They had all found this process helpful and most wanted to have the opportunity to reflect in this manner on a regular basis.

\section{Reflections on the Findings}

Although this study was small, the findings offer important new insights into the ways in which working with people experiencing suicidal ideation can impact on all aspects of the lives of mental health nurses who work with people experiencing suicidal ideation. The findings also reveal insights into how a sample group of nurses in practice understand their work with this client group and the factors that influence the ways in which this work impacts upon their lives.

The findings from this study resonate with the content of current literature in regard to the cultural and professional context in which the study took place. This is evident in the description of the sense of dissonance experienced by these particular nurses whose philosophy of practice is informed by the person centred and humanistic principles promoted by nursing proponents of this way of working (Watson, 2000; Barker, 1999; Crowe, 2000) and yet who continue to have to practice in an environment dominated by the discourse of the medical model.

The findings also resonate with the current literature in regard to concerns raised about the lack of evidenced based models of care for nurses working with people experiencing suicidal ideation (Cutcliffe \& Stevenson, 2006).

\section{Reflections on the Methodology}

The method and design of the study was informed by Sandelowski's (2000) presentation of qualitative description. This framework offered the ideal structure from which the events and phenomena under investigation could be discovered and described without being overly interpreted, thus enabling 'the voice' of the participants to be heard. Their endorsement of the findings support the claim that the aim of the study has been met.

\section{Rigour}

Evidence of dependability can be found in the clear audit trail that enabled readers to follow the decision-making processes throughout the study. Evidence of credibility can 
be found in the description of the scope of the study, the description of the setting and single location and in the discussion on the limitations of the study.

Evidence of the veracity of the findings and of the integrity of the processes is found in the systemic organisation used to manage the gathering, analysis and reporting of the data.

Confirmability was evidenced by the participants' verification that the report on the findings met Sandelowski's (2000) criterion for descriptive and interpretive validity. The quality measures adopted in this research project followed Sandelowski's recommendations and also followed commonly held principles in terms of establishing academic rigour (Minichiello, et al. 1999; Roberts \& Taylor, 2002). Evidence of this can be found in chapter three on page twenty six, in Table 1 titled, 'Design of the study and accompanying methodological rationale'.

\section{Validity}

The participants were all given a copy of the findings chapter and were asked to comment on their experiences after reading it. They were asked to comment in general and in relation to interpretive and descriptive validity. This was done prior to the drafting of this chapter, which was in keeping with the study design. In their feedback the participants spontaneously and unanimously agreed that the findings chapter accurately described their experiences.

\section{Descriptive Validity}

The participants all reported strongly believing that the findings met a high standard of descriptive validity.

A common experience for the participants was that they had all checked quotes that they had been sure was theirs only to find that it was someone else's. They had all also read a number of quotes and had thoughts such as "Yes, that's so true! Why didn't I think to say that?" (Participant four).

\section{Interpretive Validity}

The participants all reported strongly believing that the findings met a high standard of interpretive validity. 
Most appreciated the way in which the findings had been presented in terms of how the themes had been drawn from the work. Some participants reflected that they believed they too would have extracted similar themes had they been faced with this task.

They all thought that the way in which the material had been presented had enabled them to gain a fuller understanding of their work and, for most of them, this was seen as positive as it validated what they do in practice. It also had them come to appreciate the importance of they work they do as well as coming to appreciate more fully their relationships with like-minded colleagues. Two participants commented that made it likely that they would speak up in situations where previously they may have stayed silent to order to avoid conflict.

One of the participants also endorsed the high standard of interpretive validity reached in the study, but for them their reading of the findings confirmed that their recent decision to leave this area of practice had been the right one. This same participant also commented that when they read the findings, they were struck by matter of fact way in which the profound nature of the material was reported on. "You just wrote it like it is, and reading it bought it all back and I am just so glad I am out of it." (Participant one).

\section{Craig's Story}

When working with the method, as outlined in chapter three, I felt confident that I was able to be mindful of the key objectives of listening to and understanding the participants' thoughts and perceptions and of working with the texts carefully, in order to capture the key elements and present "the facts of the case in everyday language" (Sandelowski, 2000, p. 336). As the researcher, I was pleased to learn of the participants responses to reading Craig's story as this reinforced that my management of the research process had supported the conditions of validity as outlined in the method.

The participants unanimously endorsed the claim that Craig's story rendered a highly accurate portrayal of them as they worked with people experiencing suicidal ideation and that it accurately reflected the context in which they practiced. It was interesting to learn that, despite reading that the story had been composed out of the findings as a whole, three of the participants initially believed that Craig's character was based solely on them. 
One participant found it serendipitous that they had read about Craig's explanation about the importance of getting a medical review on someone who had ligature marks later on exactly the same day that they had argued in real life with someone who had not been going to facilitate a review for someone who had tried to hang themselves at their place of work.

Another was particularly moved by reading about what Craig felt as he got into bed with his wife as this had resonated very strongly with their own experiences. Until reading about this, the participant had believed that Craig's experience was something peculiar to them and that this meant there was something wrong with them. Learning that at least one of the other participants shared their experiences had caused them to see that experience was related to the extreme nature of their work.

When they were speaking of him, some participants referred to Craig as if he were a real person and most referred to him as a nice guy. Remarkably, they were unaware that they were speaking of themselves and the fact that they were the nice guys to whom they were referring.

After they had read the findings the participants all told being surprised by the scope of what had been revealed in the findings and of feeling pleased they had participated in a project they believed was worthwhile.

The participants' response to reading Craig's story validated the decision to present the findings in such a way. The participants strongly identified with Craig and appreciated the way in which the story wove the themes that were revealed in the study together into an easily read "real life" presentation of their experiences.

\section{Te Whare Tapa Wha}

The decision to use of Durie's Te Whare Tapa Wha (1998) was useful as a frame work around which to structure the aide-mémoire and to present the findings that explored the impacts on the personal lives of the participants as a result of their work with people experiencing suicidal ideation.

As the researcher, all aspects covered in the interviews were interesting and equally important to me, however it was when the participants and I explored the personal impacts of this work, that I was most moved. It could be that I identified with the participants' experiences, but mostly I believe it was because in every case the 
fundamental quality of the interviews seemed to change when we began to explore this area. In other areas of the study it seemed as if the participants and I were protected to some degree by the professional nature of the inquiry while in this part of the study it was as if that protection was no longer afforded to us. In every case, as we entered into this area of the interviews, the participants seemed to settle into a contemplative and a seemingly intentionally vulnerable state that enabled us to explore aspects of themselves that I imagine they normally keep well protected, perhaps even from themselves. Even when they were intent on describing their experiences and although they were dealing with emotionally provocative material, they seemed able to retain a degree of separation and sense of objectivity in regard to this. On reflection, I am left wondering if mine, and the participants, familiarity with the concepts of Whare Tapa Wha (Durie,1998) provided us with a safe structure in which to sit while addressing these issues and that it might also have enabled the participants to access those parts of themselves that they endeavour to access in those they are seeking to help.

\section{Limitations}

This study was limited by its size. As already acknowledged in the introduction, a small study of this size cannot achieve full data saturation so it cannot fully answer the research question. Given this, however, enough information was provided so that insights were gained into the impacts that working with people experiencing suicidal ideation have had on these particular five mental health nurses. These insights are in themselves provocative because they give rise the question of whether or not these insights are peculiar only to this group or whether they are indicative of what is true for other mental health nurses.

It could be argued that this study was limited by the fact that all of the participants by happenstance worked for the same DHB. All this means is that the findings of this study can only claim to be 'true' in regard to the experiences of these particular participants and to the particular contextual setting of this study and this is all a small exploratory study such as this can seek to claim anyway.

\section{Implications and Recommendations arising from the Discussion}

As in any exploratory study, the significance of the knowledge generated here lies in what the summary offers in terms of an end point in its self and what it offers in terms of providing a basis from which to build more knowledge upon (Sandelowski, 2000; 
Roberts \& Taylor, 2002; Minichiello et al, 1999). The next section discusses the significance of the findings and what this means in terms of practice, policy and future research.

\section{Practice}

The summary of this study offers insights into the impacts that working with people experiencing suicidal ideation has on mental health nurses. These insights are significant in that they imply that nurses are affected by, and profoundly impacted upon, in regard to almost every aspect of their lives as a result of their work with people experiencing suicidal ideation. These impacts have both negative and positive effects.

This knowledge has application in terms of practice as it may help nurses who work in similar settings recognise the phenomena described in the study and enable them to critically reflect on their own experiences in their work with people experiencing suicidal ideation and in their relationships with their colleagues. The findings from this study also offer senior nurses a useful basis from which to facilitate a critical discussion in their nursing teams. The discussion would ideally enable the team to explore whether the findings from this study apply in regard to their own place of practice. They could then discuss the implications of this in terms of team dynamics in regard to how they work with people experiencing suicidal ideation.

The findings from the study suggest that nurses who have and can articulate a personal philosophy of practice feel confident in the way they work with people experiencing suicidal ideation and that this transfers into the way in which they work in teams and to their decision making abilities in terms of risk management. I would recommend to senior nurses that they identify nurses such as these and encourage them to mentor students and new graduates and that they work with them to develop mentoring packages specific to working with people experiencing suicidal ideation. I also recommend that nurses such as these are encouraged to facilitate regular debriefing sessions in which ethical decision making and the moral imperatives that inform this process are explored from a team perspective.

\section{Policy}

The knowledge gained as a result of this study has implications in term of future policy in that it adds support to many of the concerns that have already been raised in the 
sector in regard to work place development and the future and direction of mental health nursing. I made reference in the literature chapter to concerns raised in the discussion document titled 'Mental Health Nursing and its Future: a Discussion Framework' (2006). The document focused on nine specific areas of concern in relation to the New Zealand context of mental health nursing. The findings of this study are significant when read along side of these and have led me to make the following recommendation.

\section{Recommendation}

The findings from this study suggest that further education plays a part in nurses developing philosophies of care that are humanistic and person focused. The findings also suggest that further education, combined with experience, seems to play a part in building nursing confidence, thereby building the potential for leadership by example.

The discussion document raises education as one of the key concerns for the future of mental health nursing, however, I believe that what is suggested in regard to policy for future education programmes falls short when considering the frequency and complexities of a nurse's work with people experiencing suicidal ideation. My own recommendation is that a post graduate certificate programme be set up with the purpose of concentrating solely on nurses working with people experiencing suicidal ideation. The content of the programme would include a looking at suicide from an historical and cultural perspective; looking at social, cultural, psychiatric and psychological perspectives in regard to understanding and working with suicidal people; looking at nursing and it's history and where it sits in terms of working with suicidal people; looking at the ethical decision making processes and the moral imperatives that guide nurses in practice; training in specific supervision so that nurses know how to give and receive effective supervision aimed at managing some of the impacts of this work on their personal selves; and managing the care of a person experiencing suicidal ideation with in the complex context of a multi disciplinary team.

Models of care for working with people experiencing suicidal ideation might also be included. However the findings from this study suggest that individual styles and aptitude and the capacity to build interpersonal relationships are important in nurses who work in this area of practice. 
My reasons for suggesting that this should be undertaken at a post graduate level is so nurses have some background in practice prior to undertaking a course that would be provocative in terms of the personal challenges in may present to some people and also that they would have some experience to bring for discussion purposes.

\section{Future Studies}

This is huge really. Any one of the aspects discussed here could form the basis for a large-scale research project. (Participant 2).

To start with, the findings from this study are significant because, if what was revealed here is true for the participants, the same may hold true for other nurses who work in similar settings with people experiencing suicidal ideation. Further studies would need to be carried out to confirm this hypothesis and replica studies might be a useful way of exploring this. The value of such studies would be in finding out if there were differences in the findings and, if so, what reason there was for these. This information would be useful as it could provide useful variables for comparative studies that sought to explore what these differences might mean. For example, nurses from another DHB might all base their philosophy of care on the medical model and, therefore, not experience as much conflict in their workplace. They might also have a strong leadership presence in their team so it would be hard to establish which if any one of those variables resulted in there being less conflict. A study that tested these variables may make this clear.

The knowledge gained from this exploratory study goes toward adding to what is already known by providing some insights into the impacts that working with people experiencing suicidal ideation has on mental health nurses in relation to philosophies of practice and models of care. Another study might look into the complex process of how nurses develop their personal philosophy of care and attempt to determine what factors influence this. This study has revealed the probability that education and experience are important factors, but it also suggests that individual personality structure, environmental influences and organisational and professional expectations could also be factors.

My own interest would lie in designing a participatory action research project that drew on established knowledge about clinical supervision in relation to mental health nursing 
and also drew on some of the philosophical underpinnings and frame work of Durie's Te Whare Tapa Wha (1998) in order to develop a supervision tool. This idea came from the participants who revealed that the set of interview questions used in the study had caused them to reflect on themselves and on their practice in ways that they had not done before. This was useful for them and they spoke of wanting to have more opportunities to explore themselves and their practice in this same way. The aim of developing such a tool would be to, not only help nurses in their work with people experiencing suicidal ideation, but also to encourage nurses to reflect on all aspects of themselves and, out of that, to develop strategies that minimise the negative impacts that working with people experiencing suicidal ideation has on them in relation to their personal and professional selves. A participatory action study would allow for the development and evolvement of such a tool over time and might also allow for the exploration of using the tool both individually and in groups. Developing a tool in this way would mean that the tool was tested and that it was developed from the ground up by those who would provide the supervision and those who would receive it. Ideally, a number of similar projects would be carried out simultaneously and the results compared in order to identify similarities and differences between the outcomes.

One of the recommendations made in 'Mental Health Nursing and it's Future: a Discussion Framework (2006) was that a national training model for professional supervision be developed by mental health nursing professional bodies and incorporated into standards for mental health nursing. While I believe that a national training model would be useful, I do not agree that it should be developed by mental health nursing professional bodies until there is a shared mandate on the philosophical drivers that will inform future education for mental health nursing and that will inform other aspects of practice such as models of care for working with people experiencing suicidal ideation.

\section{Reflections on the Learning Gained as a Result of undertaking this Study}

At the end of this process I can only endorse the words of Sandelowski (2000) who said "There is nothing trivial or easy about getting the facts, and the meanings the participants give to those facts, right and then conveying them in a coherent and useful manner" (p.336). My hope is that I have achieved this. 
As well as the learning gained from doing the research, I have also experienced new insights into the art of mental health nursing as it relates to working with people experiencing suicidal ideation. I am grateful to the participants for sharing their knowledge and experience and know that the essence of my own practice has been enriched as a result of this process.

\section{Conclusion}

The overall aim of this research was to describe the impact that working with people experiencing suicidal ideation has on the personal and professional lives of mental health nurses who work in acute mental health settings in New Zealand. This study has met its overall aim and has also met what Sandelowski (2000) recommended as criteria for qualitative rigour in terms of this method.

Given the nature of the enquiry, the decision to follow Sandelowski's (2000) qualitative descriptive methodology proved very apt as it enabled the participants' experiences to be reported on with low inference, thus allowing the participants' "voice" to be heard in it's own "every day language" (p.336).

The findings were powerful in that they revealed that the doing of this work has impacted on these nurses and their families in very profound ways. These findings have led to several recommendations for future studies and also have implications in terms of practice and future policy making.

The knowledge gained in this study reflects concerns that have already been raised about the future of mental health nursing at national and international levels and adds to the body of knowledge about mental health nursing in regard to the care of people experiencing suicidal ideation.

The participants in this study were very generous and extraordinarily free with themselves as nurses and with themselves as people. Without this generosity this study could not have become what it is. I feel very proud that they so emphatically validated this work as it is as much theirs as it is mine and it was from conversations with the likes of them that the idea for this work began. However, what I am most proud of is that the participants feel as though their work with people experiencing suicidal ideation can be seen in this study and be recognised as being worthwhile and important. 
I found the following piece on a wall in Mary Potter Hospice in Wellington whilst there with my father as he passed. My father's cousin bought it to my attention saying that it reminded her of my father's mother. My grandmother had been a district nurse in a remote area on the East Coast in the early1920s and made her home there after she met and married my grandfather.

Dad's cousin recalled that she had been told by her mother that my grandmother had a special gift for working with Maori who were 'unbalanced' and that she had reportedly stopped some of them from ending their lives. She told me that this work was in my blood.

I think this also reflects some of the essence of what the participants described of their work with people experiencing suicidal ideation.

\section{The Bridge}

There are times in life when we are called on to be bridges, not a great monument spanning a distance and carrying loads of heavy traffic, but a simple bridge to help one person from here to there over some difficulty such as pain and loneliness, a bridge which opens the way for ongoing journey.

When I become a bridge for another I bring upon myself a blessing, for I escape from the small prison of self and exist for a wider world, breaking out to be a larger being who can enter another's pain and rejoice in another's triumph

Joy Cowley (1989) 


\section{Appendices}

Appendix One

Appendix Two

Appendix Three
Ethics approval

Participant information sheet

Consent form 


\section{Appendix One}

TE WHARE WĀNANGA O TE ŪPOKO O TE IKA A MĀUI 9: VICTORIA

\section{MEMORANDUM}

$\begin{array}{ll}\text { Phone } & 0-4-4635676 \\ \text { Fax } & 0-4-4635209 \\ \text { Email } & \text { Allison.kirkman@vuw.ac.nz }\end{array}$

\begin{tabular}{|c|c|}
\hline TO & Lynley Murtagh \\
\hline $\begin{array}{l}\text { COPY } \\
\text { TO }\end{array}$ & Associate Professor Cheryle Moss \\
\hline FROM & Dr Allison Kirkman, Convener, Human Ethics Committee \\
\hline DATE & November 30, 2006 \\
\hline PAGES & 1 \\
\hline $\begin{array}{l}\text { SUBJEC } \\
T\end{array}$ & $\begin{array}{l}\text { Ethics Approval: No } 130 / 2006 \text {, Juggling Responses: what impact } \\
\text { does working with suicidal clients have on mental health nurses? }\end{array}$ \\
\hline
\end{tabular}

Thank you for your application for ethical approval, which has now been considered by the Standing Committee of the Human Ethics Committee.

Your application has been approved and this approval continues until 30 July 2007. If your data collection is not completed by this date you should apply to the Human Ethics Committee for an extension to this approval.

Best wishes with the research.

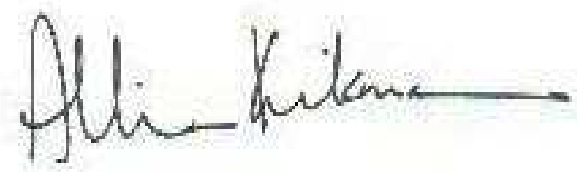

Allison Kirkman

Convener 


\section{Appendix Two}

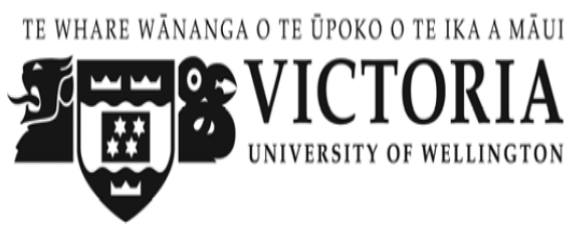

\section{Participant Information Sheet}

\section{Research Title Juggling Responses: What Impact Does Working with Clients Experiencing Suicidal Ideation Have on Mental Health Nurses?}

Researcher: Lynley Murtagh: Post Graduate School of Nursing, Midwifery and health at Victoria University in Wellington.

I am a mental health nurse and a part time Masters student in an MA (Applied) at Victoria University of Wellington. As part of this degree, I am undertaking a research project leading to a thesis. The University requires that ethics approval be obtained from research involving human subjects

I am inviting registered nurses with more than five years experience and who work with this client group, to take part in a research project that identifies how the phenomenon of working with people who are experiencing suicidal ambivalence impacts on their lives. Participants will be asked to take part in a tape recorded interview session that is expected to take approximately ninety minutes. The interview will ask you to share your stories on what may have impacted on you as a result of your work with clients experiencing suicidal ideation. The time and place for this will be arranged for your convenience. Before the interview proceeds you will be given a chance to ask questions and if you wish to proceed, you will be asked to sign a consent form.

This subject is highly sensitive and is expected to cause some emotional response. To lessen the risk of causing undue distress, I am also asking that only nurse currently undertaking clinical supervision and who have access to Employee Assistance Programmes schemes apply.

The tape recorded interviews will be transcribed and participants will be encouraged to check their interview transcripts and make alterations before the information is included in my research. The data collected will form the basis of my research project and will be put into a written report. No material that which could identify you will be included. Pseudonyms will be used for all participants and participants will be asked to refrain from using information that could identify patients.

The material collected will be kept secure and confidential. No other person besides me, the person who transcribes the interviews and my supervisor will have access to it. The tape recording of your interview will be returned to you, paper copies of the transcripts destroyed at the conclusion of the research and electronic information destroyed safely after two years. The thesis will be submitted for marking to the Post Graduate School of Nursing and Midwifery and will be deposited in the university library. I may also present the finding of the research at conferences or submit articles for publication to nursing journals. You will be offered a copy of the completed thesis.

Taking part is entirely voluntary and participants may withdraw from the project at any time before the data is analysed without giving a reason.

If you have any questions or would like to receive further information abut the project, please contact me; Lynley Murtagh ph.027 4423186 or email lynley.murtagh@ccdhb.org.nz ,

Or

My supervisor; Associate Professor Cheryle Moss, at the Post Graduate School of Nursing, Midwifery and Health at Victoria University, Wellington. PO box 600 Wellington, ph 4636141. 


\section{Appendix Three}

\section{Consent to Participation in Research}

\section{Title of project: Juggling Responses: What Impact Does working With Clients Experiencing Suicidal Ideation Have on Mental Health Nurses?}

\section{Researcher; Lynley Murtagh}

I have been given and have understood an explanation of the nature and objectives of this research project.

I have had an opportunity to ask questions and have had them answered to my satisfaction.

I understand that taking part in this study is voluntary and that I may withdraw myself (or any information I have provided) from this project (before analysis has begun) without giving a reason and that any data I have provided will be returned to me.

I understand that any information I give will be kept confidential to the researcher, the supervisor and the person who transcribes the tape recordings of our interview.

I understand that the information I have provided will be used only for this research project.

The published results will not use my name and no opinions will be attributed to me in any way that will identify me.

I understand that the tape recordings will be returned to me at the conclusion of the research and that all other data will either be destroyed or kept secure for up to two years.

I will be offered a copy of the completed thesis at the conclusion of this research.

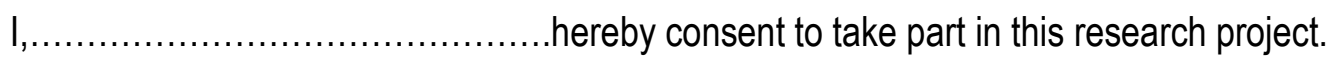

Signature.

Full name of researcher

Contact phone number of researcher

Signature.

Date.../............. 


\section{Reference List}

Alexander, D.A., Klein, S., Gray, N.M., Dewar, I. G., \& Eagles, J. M. (2000). Suicide by patients: A questionnaire study of its effects on consultant psychiatrists. British Medical Journal, 320, 1571-1574.

Alston, M. H., \& Robinson, B H. (1992). Nurses attitudes toward suicide. Omega, 25(3), 205-215.

Associate Minister of Health. (2006). The New Zealand Suicide Prevention Strategy 2006-2016. Wellington: Ministry of Health.

Barker, P. (1999). The philosophy and practice of psychiatric nursing. Edinburgh: Churchill Livingston

Barker, P. (2001). The tidal model; Developing a person-centred approach to recovery within psychiatric and mental health nursing. Journal of Psychiatric and Mental Health Nursing, 8(3), 233-240.

Barker, P. (2006). 30 ${ }^{\text {th }}$ Anniversary commentary on Crowe M. (2000) Psychiatric Diagnosis: Some implications for mental health nursing care. Journal of Advanced Nursing 31(30), 583-589.

Barker, P., \& Cutchcliffe, J.R. (1999). Clinical risk; A need for engagement not observation. Mental Health Practice, 2(8) 8-12.

.Berg, B. (1995). Qualitative research methods for the social sciences $\left(3^{\text {rd }}\right.$ ed). Boston: Allyn \& Bacon.

Bishop, D., \& Ford-Bruins, I. (2003).Nurses' perceptions of mental health assessment in an acute inpatient setting in New Zealand: A qualitative study. International Journal of Mental Health Nursing, 12(3), 203-212.

Brink, P., \& Wood, M. (1994). Basic steps in planning research. London: Jones \& Barlett.

Clark, M.L., \& Gioro, S. (1998). Nurses, indirect trauma and prevention. The Journal of Nursing Scholarship, 30(1),85-87.

Clegg, A (2001). Occupational stress in Nursing: A review of the literature. Journal of Nursing Management, 9(2) 101-106. 
Coddington, D. (2005, May 15). It's the sorrow that dare not speak its name. Herald on Sunday, p.35.

Collings, S. \& Beautrais, A., (2005). Suicide prevention in New Zealand: A contemporary perspective. Wellington: Ministry of Health.

Cook, N.R., Phillips, B.N., \& Sadler, D. (2005). The Tidal Model as experienced by patients and nurses in a regional forensic unit. Journal of psychiatric and mental health nursing, 12(5), 536-40.

Cowley, J. (1989). Aotearoa Psalms. Otane: Pleroma Press.

Crotty , M. (1998). The foundations of social research: meaning and perspectives in the research process. London: Sage.

Crowe, M, (2000). Psychiatric Diagnosis: Some implications for mental health nursing care. Journal of Advanced Nursing, 31(3), 583-589.

Crowe, M. (1997). The socio-political context of mental health nursing. Australian and New Zealand Journal of Mental health Nursing, 6, 59-65.

Crowe, M., O’Malley, J., \& Gordon, S. (2001). Meeting the needs of consumers in the community: A working partnership in mental health in New Zealand. Journal of Advanced Nursing, 35(1), 88-96.

Cutcliffe, J.R., \& Stevenson, C. (2008). Feeling our way in the dark: The psychiatric nursing care of suicidal people- A literature review. International Journal of Nursing Studies 45, 942-953.

Deardon, G. (2004). When things go wrong: The experiences of mental health nurses who have had a patient die through suicide. Unpublished master's thesis, Victoria University of Wellington, Wellington, New Zealand.

Denzin, N.K., \& Lincoln, Y.S. (Eds.) (2000). Handbook of Qualitative Research. (2 $^{\text {nd }}$ ed) Thousand Oaks, CA: Sage.

Denzin, N.K., \& Lincoln, Y.S. (Eds.) (2008). Collecting and interpreting qualitative materials. $\left(3^{\text {rd }}\right.$ ed) Thousand Oaks, CA: Sage.

Duffy, D. (1995). Out of the shadows, a study of special observation of suicidal inpatients. Journal of Advanced Nursing, 21, 944-950. 
Durie, M. (1998). Whaiora: Maori health development $\left(2^{\text {nd }}\right.$ ed) Auckland: Oxford University Press.

Fletcher, R.F. (1999). The process of constant observations: Perspectives of staff and suicidal patients. Journal of Psychiatric and Mental Health Nursing, 6(1), 9-14.

Firestone, R. W. (1997). Suicide and the inner voice; Risk assessment, treatment and case management. California: Sage.

Goldney, R. (2001). The Media and suicide: A cautionary view. Crisis, 22(4), 173-175.

Hapete, H. (2005, May 5). Why make the pain of suicide public? Otago Daily Times

Happell, B., Pinikahana, J., \& Martin, T. (2003). Stress and burnout in forensic psychiatric nursing. Stress and health: Journal of the international society for the investigation of stress, 19(2), 63-68.

Health Workforce Advisory Committee, (2002). The New Zealand Health Workforce: Framing future directions discussion document. Wellington: Author

Healy, C., \& McKay, C. (2000). Nursing stress: The effects of coping strategies and job satisfaction in a sample of Australian nurses. Journal of advanced nursing, 31(3), 681-688.

Heron, J. (1990). Helping the client. A creative and practical guide. London: Sage.

Hillman, J. (1997). Suicide and the soul ( $2^{\text {nd }}$ ed). Putman: Spring.

Hawthorne, K., \& Williams, K. (2001). The connection between media and suicidal behaviour warrants serious attention. Crisis, 22(4), 137-140.

Iveson, C. (2002). Solution focused brief therapy. Advances in Psychiatric Treatment, 8, 149-156.

Jamison, K., R. (2000). Night falls fast: Understanding suicide. Oxford: Picador.

King, M. (2003). The Penguin history of New Zealand. Auckland: Penguin.

Langdon, C. (2000, November 11). Mental health boss backs his assessment team. Dominion Post .p 2. 
Lauder, W., Forest, S.,\& Masters, H. (2006).The national review of mental health nursing in Scotland: Implications for the profession. Journal of Psychiatric and Mental Health Nursing, 13(6), 634-635.

Lincoln, Y., \& Guba, E. G. (1985). Naturalistic Inquiry, Beverley Hills: Sage.

Luke, B. (2004). Achieving a mind body spirit connection: A stress management workbook. Boston: Jones \& Bartlett.

Mann, S., \& Cowburn, J. (2005). Emotional labour and stress within mental health nursing. Journal of Psychiatric and Mental Health Nursing, 12(2), 154-162.

McCann, I.L., \& Pearlman, L.A. (1990). Vicarious traumatization: A framework for understanding the psychological effects of working with victims. Journal of Traumatic Stress, 3, 131-149.

Martin, C.A., McKean, H.E., \& Veltkamp, L.J. (1994). Post traumatic stress disorder in Police and working with victims: A pilot study. Journal of Police Science and Administration, 14, 98-101.

Maslow, A. H. (1968). Toward a psychology of being (2 $2^{\text {nd }}$ ed). New York: Van Nostrand Reinhold.

Mental Health Commission. (2001). Open all hours?: A review of crisis mental health services. Wellington: Author.

Minichiello, V., Sullivan, G., Greenwood, K. \& Axford, R. (1999). Handbook for research methods in health sciences. Sydney: Addison-Wesley.

Ministry of Health, (2002). Suicide and the media: The reporting and portrayal of suicide in the media. A resource. Wellington: Author.

Ministry of Health, (2006). Mental Health Nursing and its future: a Discussion framework. Wellington: Author.

Ministry of Youth Affairs, Ministry of Health, Te Puni Kokiri.1998. The New Zealand Youth Prevention Strategy; "In Our Hands" and "Kia Piki te Ora o te Taiamariki" Wellington: Ministry of Youth Affairs, Ministry of Health and Te Puni Kokiri.

Morse, J.M.(1994). Designing funded qualitative research. In N.K. Denzin, \& 
Y.S. Lincoln, (Eds). Handbook of qualitative research (pp.220-235). Thousand Oaks, California: Sage.

O'Brien A.J., Thom. K., \& Hughes, F. H. (2007). Acute mental health nursing: A discussion document. Auckland: Centre for Mental Health Research.

Patton, M Q. (1990). Qualitative evaluation and research methods. Newbury Park: Sage.

Peplau, H. (1988). Interpersonal relations in nursing. A conceptual frame of reference for psychodynamic nursing. London: McMillan.

Pryjmachuk, S., \& Richards, D. (2007). Mental health nursing students differ from other nursing students: Some observations form a study on stress and coping. International Journal of Mental Health Nursing, 16(6), 390-402.

Raphael, B., \& Wilson, J.P. (Eds.). (2000) Psychological debriefing; Theory, practice and evidence. Cambridge: University Press.

Richardson, A. (2005, September 3). The Poor Relative. Listener, 24-26.

Roberts, A. R. (Eds). (2000). Crisis intervention handbook: Assessment, treatment and research $\left(2^{\text {nd }}\right.$ ed). Oxford: University Press.

Roberts, K., \& Taylor, B. (2002). Nursing research processes: An Australian perspective ( $\left.2^{\text {nd }} \mathrm{ed}\right)$. Victoria: Thomson.

Rogers, C. (1986). A client centred/person centred approach to therapy. In $\mathrm{H}$. Kirshenbaum, and V. Henderson (Eds.). The Carl Rogers Reader. London: Constable.

Ryan, D., \& Quayle, E. (1999). Stress in psychiatric nursing: Fact or fiction? Nursing Standard.14(8), 32-35.

Sandelowski, M. (1986). The problems of rigour in qualitative research. Advances in Nursing Science, 8, 27-37.

Sandelowski, M. (1993). Rigor or rigor mortis? The problems of rigour in qualitative research revisited. Advances in Nursing Science, 8, 27-37.

Sandelowski, M. (2000). What ever happened to qualitative description? Research in Nursing and Health, 23, 334-340. 
Steed, L.G., \& Downing, R. (1998). A phenomenological study of vicarious traumatisation among psychologists and professional counsellors working in the field of sexual abuse/assault. The Australian Journal of Disaster and Trauma Studies, Volume:2.

Stordeur, S., D'Hoore, W., \& Vandenberghe, C. (2001). Leadership, organisational stress, and emotional exhaustion among hospital nursing staff. Journal of advanced nursing 35(4), 53-542.

Sudak, H., \& Sudak, D. (2005). The media and suicide. Academic Psychiatry, 29, 495499

Suicide. (n.d) Retrieved October 25 2008, from http://enwikipedia.org

Tully, J., \& Elsaka, N. (2004). Suicide and the media, A study of the media response to suicide and the media: The reporting and portrayal of suicide in the media. A resource. School of Political Science and Communication: University of Canterbury, Christchurch.

Van den Tooren, M.J. (2008). Managing job stress in nursing: What kind of resources do we need? Journal of Advanced Nursing, 63(1) 75-84.

Watson, P. (2002,) Mental health nursing: The art of compassionate care. Edinburgh; Butterworth-Heinemann.

Wolcott, H.F. (1994). Transforming qualitative data: Description, analysis and interpretation. Thousand Oaks, CA :Sage. 